Review

\title{
Marine Indole Alkaloids
}

\section{Natalie Netz and Till Opatz *}

Institute of Organic Chemistry, Johannes Gutenberg-University Mainz, Duesbergweg 10-14, 55128 Mainz, Germany; E-Mail: netz@uni-mainz.de

* Author to whom correspondence should be addressed; E-Mail: opatz@uni-mainz.de; Tel.: +49-6131-39-24443; Fax: +49-6131-39-22338.

Academic Editor: Valeria Costantino

Received: 30 June 2015 / Accepted: 24 July 2015 / Published: 6 August 2015

\begin{abstract}
Marine indole alkaloids comprise a large and steadily growing group of secondary metabolites. Their diverse biological activities make many compounds of this class attractive starting points for pharmaceutical development. Several marine-derived indoles were found to possess cytotoxic, antineoplastic, antibacterial and antimicrobial activities, in addition to the action on human enzymes and receptors. The newly isolated indole alkaloids of marine origin since the last comprehensive review in 2003 are reported, and biological aspects will be discussed.
\end{abstract}

Keywords: indoles; alkaloids; marine natural products; bisindoles; nitrogen heterocycles; carbolines; prenylated indoles; diketopiperazines

\section{Introduction}

Alkaloids represent a large and highly structurally diverse group of secondary metabolites. The presence of nitrogen in their molecular architecture confers biological activity to an exceptionally large fraction of this compound class. Therefore, it comes as no surprise that mammals-including man-have acquired the ability to detect the potentially toxic alkaloids by their bitter taste.

As the origin of life on Earth presumably was the early hydrosphere, the evolution of aquatic life forms has the longest history and a connection may be seen in the enormous chemical complexity of natural marine products.

This review focuses on marine indole alkaloids, discovered since the last comprehensive report by Aygün and Pindur in 2003 [1]. In addition to structures and occurrence, known biological activities of 
marine indole alkaloids will be discussed. We will make use of Pelletier's general definition of 1983, according to which alkaloids are "cyclic organic compounds containing nitrogen in a negative oxidation state which are of limited distribution among living organisms" [2]. As an additional demarcation against the world of peptides, polypeptidic structures and macrocyclic peptides derived from tryptophan, such as terpeptins [3] and related structures [4], milnamides [5,6], diazonamides [7], lucentamycin B [8], pipestelides [9], kahalalides [10-12], jaspamides [13], jasplakinolides [14], etc., will not be discussed here. Indole alkaloids which were isolated from genetically engineered marine derived organisms, from organisms with an artificially altered gene regulation, or which were obtained through genetic engineering of terrestrial organisms using genes of marine organisms will not be discussed in this review [15-21].

The indole nucleus is one of the most important ring systems for pharmaceutical development and has been termed a "privileged structure" in this respect [22]. It is frequently associated with the action on G-protein coupled receptors, in particular with the modulation of neuronal signal transmission through receptors for serotonin (5-hydroxytryptamine, 5-HT). A large variety of effects on other molecular targets have also been reported, including glycine-gated chloride channel receptors, human protein tyrosine phosphatase-1B, the CXCR4 (C-X-C chemokine receptor type 4 ) chemokine receptor, $\mathrm{Na}^{+} / \mathrm{K}^{+}$-ATPase, nitric oxide synthase, $\beta$-secretase, protein kinase $\mathrm{C}-\alpha$, butyrylcholinesterase, and acetylcholinesterase. Furthermore, cytotoxic, antineoplastic, antibacterial, antifungal, antiinsecticidal, and antiplasmodial activities have been detected.

Apart from its capacity to act as a hydrogen bond donor through a free NH function, the high $\pi$-electron density and the high HOMO (highest occupied molecular orbital) energy of the planar indole skeleton permit interactions with nucleobases - in particular protonated ones - as well as target proteins, some of which exhibit a high binding specificity for the indole nucleus. The electronic properties together with the relatively low resonance energy of the five-membered ring also determine the chemical behavior of indoles and many of their derivatives. Thus, electrophilic substitutions or oxidative transformations, partly under loss of the aromatic stabilization, are paramount for this compound class, which is also reflected by the structures of many of the compounds discussed in this review.

Regarding related work, an overview of the biosynthesis of indole alkaloids from fungal origin has been published by Xu et al. [23]. Alkaloids from marine algae are discussed by Güven et al. [24] and halogenated indole alkaloids from marine invertebrates have been reviewed by Pauletti et al. [25].

\section{Marine Indole Alkaloids}

\subsection{Simple Indole Alkaloids}

The simple indole alkaloids are mostly derived from tryptophan or its direct precursor indole, which itself is formed from chorismate through anthranilate and indole-3-glycerol-phosphate in microorganisms and plants. As the ultimate step of the tryptophan biosynthesis is reversible, free indole can also be formed in this catabolic process [26]. Electrophilic substitutions with iodine and especially bromine are frequently encountered in this and other subclasses presented here while the 
even more common prenylated indoles, with and without halogen substituents, will be discussed in a separate section.

$N$-3'-Ethylaplysinopsin (1) was obtained from the Jamaican sponge Smenospongia aurea and exhibited a high affinity for 5- $\mathrm{HT}_{2 \mathrm{~A}}$ and 5- $\mathrm{HT}_{2 \mathrm{C}}$ receptors (Figure 1) [27]. 6-Bromo-1'-hydroxy-1',8dihydroaplysinopsin (2), 6-bromo-1'-methoxy-1',8-dihydroaplysinopsin (3), 6-bromo-1'-ethoxy-1',8dihydroaplysinopsin (4), (-)-5-bromo- $N, N$-dimethyltryptophan (5), (+)-5-bromohypaphorine (6) and 6-bromo-1H-indole-3-carboxylic acid methyl ester (7) were obtained by investigation of an NCI-DTP (National Cancer Institute, Developmental Therapeutics Program) collection of Thorectandra sp. and a UCSC (University of California, Santa Cruz) collection of Smenospongia sp. They show weak inhibitory activity towards Staphylococcus epidermidis [28]. Halgerdamine (8) and $\mathrm{C}^{2}$ - $\alpha$-Dmannosylpyranosyl-L-tryptophan (9) were isolated from the nudibranch mollusc Halgerda aurantiomaculata. $\mathrm{C}^{2}$ - $\alpha$-D-Mannosylpyranosyl-L-tryptophan (9) was isolated from a mollusc for the first time [29].<smiles>CC/N=C1\N(C)C(=O)/C(=C\c2c[nH]c3ccccc23)N1C</smiles>

(1) N-3‘-ethylaplysinopsin<smiles>CN(C)[C@@H](Cc1c[nH]c2ccc(Br)cc12)C(=O)O</smiles>

(5)<smiles>[NH3+][C@H](Cc1c(CCO)[nH]c2ccccc12)C(=O)[O-]</smiles>

(8) halgerdamine<smiles>[R7]c1ccc2c(CC3([R])C(=O)N(C)C(=N)N3C)c[nH]c2c1</smiles>

(2) $\mathrm{R}^{1}=\mathrm{Br}, \mathrm{R}^{2}=\mathrm{OH}$

(3) $\mathrm{R}^{1}=\mathrm{Br}, \mathrm{R}^{2}=\mathrm{OMe}$

(4) $\mathrm{R}^{1}=\mathrm{Br}, \mathrm{R}^{2}=\mathrm{OEt}$<smiles>C[N+](C)(C)[C@H](Cc1c[nH]c2ccc(Br)cc12)C(=O)[O-]</smiles>

(6)<smiles>COC(=O)c1c[nH]c2cc(Br)ccc12</smiles>

(7)<smiles>N[C@@H](Cc1c([C@@H]2O[C@H](CO)[C@@H](O)[C@H](O)[C@H]2O)[nH]c2ccccc12)C(=O)[O-]</smiles>

(9) $C^{2}$ - $\alpha$-D-mannosylpyranosyl-L-tryptophan

Figure 1. Simple indole derivatives.

5,6-Dibromo-L-hypaphorine (10) has been isolated from the South Pacific marine sponge Hyrtios sp. and displays weak inhibitory effects against bee venom phospholipase $\mathrm{A}_{2}$ (IC50-inhibitory concentration to effect a $50 \%$ inhibition $-0.2 \mathrm{mM}$ ) and a significant antioxidant activity (Figure 2) [30]. Purpuroines A-J were isolated from the marine sponge Iotrochota purpurea, purpuroine $\mathrm{J}$ (6-bromohypaphorine methylate, 11) being the only indole-derived congener [31]. Iodinated 
plakohypaphorines A-F (12-17) have been isolated from the Caribbean sponge Plakortis simplex. Diiodinated species plakohypaphorines B (13), C (14) and D (15) exhibited significant antihistaminic activity $[32,33]$.<smiles>C[N+](C)(C)C(Cc1c[nH]c2cc(Br)c(Br)cc12)C(=O)[O-]</smiles>

(10) 5,6-dibromo-L-hypaphorine<smiles>[R]c1cc2c(CC(C(=O)[O-])[N+](C)(C)C)c[nH]c2c(I)c1[R]</smiles>

(12) $R^{1,2}=H$ plakohypaphorine $A$

(13) $R^{1}=H, R^{2}=$ I plakohypaphorine $B$

(14) $R^{1}=I, R^{2}=H$ plakohypaphorine $C$<smiles>COC(=O)[C@H](Cc1c[nH]c2cc(Br)ccc12)N(C)C</smiles>

(11) purpuroine $\mathrm{J}$<smiles>[R]c1c(I)cc2c(CC(C(=O)[O-])[N+](C)(C)C)c[nH]c2c1[R]</smiles>

(15) $R^{1}=I, R^{2}=$ H plakohypaphorine $D$

(16) $R^{1,2}=$ I plakohypaphorine $E$

(17) $\mathrm{R}^{1}=\mathrm{H}, \mathrm{R}^{2}=\mathrm{Cl}$ plakohypaphorine $\mathrm{F}$

Figure 2. 5,6-Dibromo-L-hypaphorine, purpuroine $\mathrm{J}$ and plakohypaphorines A-F.

Examination of the organic extract of the broth of an unidentified algicolous fungus collected from the surface of the marine red alga Gracilaria verrucosa led to the isolation of $N_{\mathrm{b}}$-acetyltryptamine (18) (Figure 3) [34]. Analysis of the Red Sea marine sponge Hyrtios erectus led to the isolation of 5-hydroxy-1H-indole-3-carboxylic acid methyl ester (19), indole-3-carbaldehyde (20) and 5-deoxyhyrtiosine A (21) [35,36].<smiles>CC(=O)NCCc1c[nH]c2ccccc12</smiles>

(18) $N_{\mathrm{b}}$-acetyltryptamine<smiles>COC(=O)c1c[nH]c2ccc(O)cc12</smiles><smiles>O=Cc1c[nH]c2ccccc12</smiles>
(20) indole-3-carbaldehyde 3-carboxylic acid methyl ester<smiles>O=C(OCO)c1c[nH]c2ccccc12</smiles>

(21) 5-deoxyhyrtiosine A

Figure 3. Simple indole derivatives.

Bacillamide A (22) was obtained from the marine bacterium Bacillus sp. SY-1 and displayed algicidal activity against Cochlodinium polykrikoides (Figure 4) [37]. Bacillamides A-C (22-24) were found in a Bacillus endophyticus isolate SP31 [38]. 
<smiles>CC(=O)c1nc(C(=O)NCCc2c[nH]c3ccccc23)cs1</smiles>

(22) bacillamide $A$<smiles>[R]C(C)c1nc(C(=O)NCCc2c[nH]c3ccccc23)cs1</smiles>

(23) $\mathrm{R}=\mathrm{OH} \quad$ bacillamide $\mathrm{B}$

(24) $\mathrm{R}=$ NHAc bacillamide $\mathrm{C}$

Figure 4. Bacillamides A-C.

6-Hydroxydiscodermindole (25) was obtained from the deep-water sponge Discodermia polydiscus and showed weak cytotoxic effects towards the murine leukemia cell line P388 (Figure 5) [39].<smiles>NC1=NC(c2c(Br)[nH]c3cc(O)c(Br)cc23)CN1</smiles>

(25) 6-hydroxydiscodermindole

Figure 5. 6-Hydroxydiscodermindole.

2-Methylsulfinyl-3-methylthio-4,5,6-tribromoindole (26), 3-methylsulfinyl-2,4,6-tribromoindole (27) and 4,6-dibromo-2,3-di(methylsulfinyl)indole (28) were isolated from the Formosan red alga Laurencia brongniartii (Figure 6) [40]. 2,3,4,6-Tetrabromo-1-methyl-1H-indole (29) was isolated from the marine red alga Laurencia decumbens [41]. 3,5,6-Tribromo-1H-indole (30), 3,5,6-tribromo-1methyl-1H-indole (31), 2,3,6-tribromo- $1 H$-indole (32), 3,5-dibromo-1-methyl-1H-indole (33) and 2,5dibromo-1-methyl-1H-indole (34) have been isolated from the marine red alga Laurencia similis [42-44]. 6-Bromo-1H-indole-3-carboxamide (35) was obtained from the marine sponge Mycale fibrexilis [45].

Dysinosins B-D (37-39) were isolated from the Australian sponge Lamellodysidea chlorea [46], while dysinosin A (36) [47] was obtained from an Australian sponge of the family Dysideidae (Figure 7). Dysinosins A-D exhibit inhibitory activity towards factor VIIa $\left(K_{\mathrm{i}}\right.$ - dissociation constant-values $0.108,0.090,0.124$ and $1.320 \mu \mathrm{M}$, respectively) and thrombin $\left(K_{\mathrm{i}} 0.452,0.170,0.550\right.$ and $>5.1 \mu \mathrm{M}$, respectively), with desulfated dysinosin $\mathrm{D}(\mathbf{3 9})$ being ten times less active $[46,47]$.

Granulatamides A (40) and B (41) were isolated from the soft coral Eunicella granulate and showed cytotoxic activity against the tumor cell lines DU-145, LNCaP, SK-OV-3, IGROV, IGROV-ET, SK-BR3, SK-MEL-28, HMEC1, A549, K562, PANC1, HT-29, LOVO, LOVO-DOX, HeLa and HeLa-APL (GI 50 concentration which effects a growth inhibition of 50\%-1.7-13.8 $\mu \mathrm{M}$ ) (Figure 8) [48]. 
<smiles>[R]c1[nH]c2cc(Br)c([R])c(Br)c2c1[R]</smiles>

(26) $\mathrm{R}^{1}=\mathrm{Br}, \mathrm{R}^{2}=\mathrm{SMe}, \mathrm{R}^{3}=\mathrm{S}(\mathrm{O}) \mathrm{Me}$

(27) $\mathrm{R}^{1}=\mathrm{H}, \mathrm{R}^{2}=\mathrm{S}(\mathrm{O}) \mathrm{Me}, \mathrm{R}^{3}=\mathrm{Br}$

(28) $R^{1}=H, R^{2,3}=S(O) M e$<smiles>Brc1ccc2c(Br)c(Br)[nH]c2c1</smiles>

(32)<smiles>Cn1c(Br)c(Br)c2c(Br)cc(Br)cc21</smiles>

(29)<smiles>Brc1cc2[nH]cc(Br)c2cc1Br</smiles>

(30)<smiles>Cn1cc(Br)c2cc(Br)c(Br)cc21</smiles>

(31)<smiles>Cn1cc(Br)c2cc(Br)ccc21</smiles>

(33)<smiles>Cn1c(Br)cc2cc(Br)ccc21</smiles>

(34)<smiles>NC(=O)c1c[nH]c2cc(Br)ccc12</smiles>

(35)

Figure 6. Simple brominated indole derivatives.<smiles>CO[C@H](C(=O)N[C@@H](CC(C)C)C(=O)N1[C@H](C(=O)NCCC2=CC[N+](=C(N)N)C2)C[C@@H]2C[C@H](O)[C@@H](O)C[C@H]21)[C@H](O)O[Na]</smiles>

(36) dysinosin A

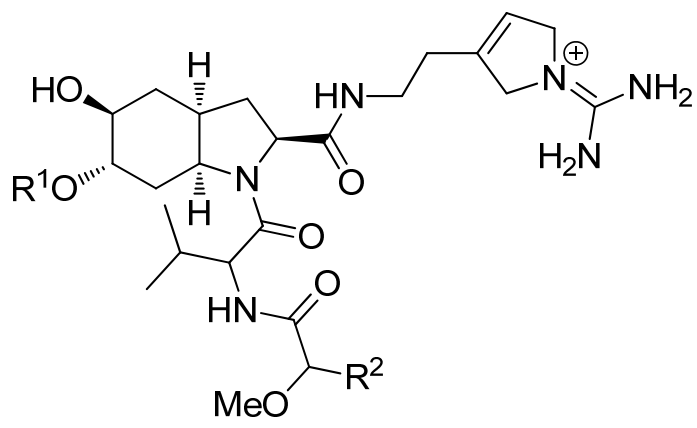

(37) $\mathrm{R}^{1}=\alpha$-glucopyranoside $\mathrm{R}^{2}=\mathrm{OSO}_{3}^{\ominus}$ dysinosin $\mathrm{B}$

(38) $\mathrm{R}^{1}=\mathrm{H}, \mathrm{R}^{2}=\mathrm{OSO}_{3}^{\Theta}$ dysinosin $\mathrm{C}$

(39) $\mathrm{R}^{1}=\mathrm{H}, \mathrm{R}^{2}=\mathrm{OH} \quad$ dysinosin $\mathrm{D}$

Figure 7. Dysinosins A-D.<smiles>CCCCCCCCC/C(C)=C/C(=O)NCCc1c[nH]c2ccccc12</smiles>

(40) granulatamide A

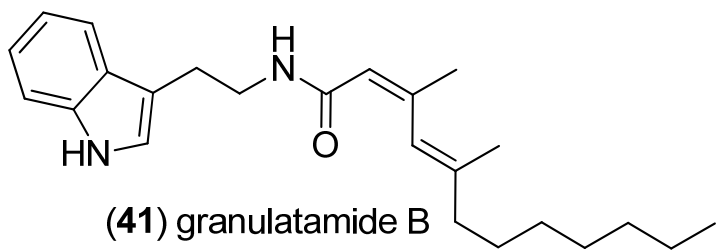

Figure 8. Granulatamides A and B.

Meridianins F (42) and G (43) have been isolated and identified from the tunicate Aplidium meridianum by tandem mass spectrometry (Figure 9) [49]. Analysis of the extract of the related tunicate Aplidium cyaneum, collected in Antarctica, afforded bromoindole derivatives, aplicyanins A-F (44-49). N-Acetylated aplicyanins B, D and F were found to have potent antimitotic, as well as cytotoxic, activities against the tumor cell lines HT-29 (GI50 0.39, 0.33, and $0.47 \mu \mathrm{M}$, respectively), A549 (GI G $_{50} 0.66,0.63$, and $1.31 \mu \mathrm{M}$, respectively) and MDA-MB-231 (GI50 0.42, 0.41, and $0.81 \mu \mathrm{M}$, respectively), while aplicyanin E showed only mild cytotoxic effects (GI50 values 7.96-8.70 $\mu \mathrm{M})$ [50,51]. 
<smiles>Nc1nccc(-c2c[nH]c3cc(Br)c(Br)cc23)n1</smiles>

(42) meridianin F<smiles>Nc1nccc(-c2c[nH]c3ccccc23)n1</smiles>

(43) meridianin $G$<smiles>[R1]N=C1NCCC(c2cn([R])c3cc([R3])c(Br)cc23)N1</smiles>

(44) $R^{1,2,3}=H$ aplicyanin $A$

(45) $R^{1}=A c, R^{2,3}=H$ aplicyanin $B$

(46) $\mathrm{R}^{1}=\mathrm{OMe}, \mathrm{R}^{2,3}=\mathrm{H}$ aplicyanin $\mathrm{C}$

(47) $R^{1}=A c, R^{2}=$ OMe, $R^{3}=H$ aplicyanin $D$

(48) $\mathrm{R}^{1}=\mathrm{H}, \mathrm{R}^{2}=\mathrm{OMe}, \mathrm{R}^{3}=\mathrm{Br}$ aplicyanin $\mathrm{E}$

(49) $R^{1}=A c, R^{2}=$ OMe, $R^{3}=B r$ aplicyanin $F$

Figure 9. Meridianins $\mathrm{F}$ and $\mathrm{G}$ and aplicyanins $\mathrm{A}-\mathrm{F}$.

Oxazinins 1-6 (50-55) and their linear precursor preoxazinin-7 (56) were isolated from toxic edible mussels (Mytilus galloprovincialis) from the Northern Adriatic coast (Figure 10). The stereochemistry of oxazinins 1 and 2 was revised to $2 R[52-55]$.<smiles>[R]Oc1ccc([C@H]2O[C@H](c3c[nH]c4ccccc34)C(=O)N[C@@H]2CO)cc1</smiles>

(50) $\mathrm{R}=\mathrm{CH}_{2} \mathrm{CH}_{2} \mathrm{CN}$ oxazinin-1 (51) $\mathrm{R}=\mathrm{H}$ oxazinin-2<smiles>O=C1N[C@H](Cc2ccc(O)cc2)CO[C@H]1c1c[nH]c2ccccc12</smiles>

(52) oxazinin-3<smiles>N#CCCOc1ccc(C2O[C@@H](c3c[nH]c4ccccc34)C(=O)N[C@H]2CO)cc1</smiles>

(53) oxazinin-4<smiles>N#CCCOc1ccc(C(O)[C@H]2CO[C@H](c3c[nH]c4ccccc34)C(=O)N2)cc1</smiles><smiles>N#CCCOc1ccc([C@@H](O)[C@H](CO)NC(=O)C(=O)c2c[nH]c3ccccc23)cc1</smiles>

(56) preoxazinin-7

Figure 10. Oxazinins 1-7.

Monoindole derivatives (57-63) were isolated from the marine sponge Spongosorites sp. by bioactivity-guided fractionation (Figure 11). Compound 63 displayed weak cytotoxic activity towards several human cancer cell lines. Those compounds, mostly indole pyruvic acid derivatives, are hypothetical biosynthetic precursors of co-occurring bisindoles, such as the hamacanthins and the topsentins, see Figure 98 [56]. 6-Bromo-5-hydroxyindolyl-3-glyoxylate ethyl ester (64) was isolated from the far eastern ascidian Syncarpa oviformis [57]. 5-Hydroxy- $1 H$-indole-3-carboxylic acid ethyl ester (65) and 5-hydroxy-1H-indole-3-glyoxylate ethyl ester (66) were isolated from the marine sponge Ircinia sp., Irciniidae, collected at Iriomote Island [58]. 
<smiles>[R]C(=O)c1c[nH]c2cc([R])ccc12</smiles>

(57) $\mathrm{R}^{1}=\mathrm{Br}, \mathrm{R}^{2}=\mathrm{CO}_{2} \mathrm{Me}$

(58) $\mathrm{R}^{1}=\mathrm{H}, \mathrm{R}^{2}=\mathrm{CO}_{2} \mathrm{Me}$

(59) $\mathrm{R}^{1}=\mathrm{OH}, \mathrm{R}^{2}=\mathrm{CO}_{2} \mathrm{Me}$

(60) $\mathrm{R}^{1}=\mathrm{H}, \mathrm{R}^{2}=\mathrm{CONH}_{2}$

(61) $\mathrm{R}^{1}=\mathrm{Br}, \mathrm{R}^{2}=\mathrm{CONH}_{2}$

(62) $\mathrm{R}^{1}=\mathrm{OH}, \mathrm{R}^{2}=\mathrm{OMe}$

(63) $\mathrm{R}^{1}=\mathrm{H}, \mathrm{R}^{2}=\mathrm{OMe}$<smiles>CCOC(=O)C(=O)c1c[nH]c2cc(Br)c(O)cc12</smiles>

(64) 6-bromo-5-hydroxyindolyl-3- (65) 5-hydroxy-1H-indole-3-carboxylic glyoxylate ethyl ester acid ethyl ester<smiles>CCOC(=O)C(=O)c1c[nH]c2ccc(O)cc12</smiles>

(66) 5-hydroxy-1H-indole-3-glyoxylate ethyl ester

Figure 11. Indole-3-glyoxylates and indole-3-carboxylates.

Iodinated heterocycles hicksoanes A-C (67-69) are derived from the gorgonian Subergorgia hicksoni and show antifouling effects (Figure 12) [59].<smiles>[R]c1cc([R])c2c(c1)NCC2C[C@@H]1NC(=O)[C@H]([C@H](C)CC)NC(C)(C)NC1=O</smiles>

(67) $\mathrm{R}^{1}=\mathrm{H}, \mathrm{R}^{2}=$ I hicksoane $A$

(68) $R^{1}=I, R^{2}=H$ hicksoane $B$

(69) $R^{1,2}=$ I hicksoane $C$

Figure 12. Hicksoanes A-C.

8,9-Dihydrobarettin (70) and bromobenzisoxazolone barettin (71), natural products with entirely unprecedented benzisoxazolone substructure were reported as isolates from the marine sponge Geodia barretti and showed inhibitory effects on the settlement of the barnacle larvae Balanus improvises (Figure 13) $[60,61]$.<smiles>N=C([NH3+])NCCCC1NC(=O)C(Cc2c[nH]c3cc(Br)ccc23)NC1=O</smiles>

(70) 8,9-dihydrobarettin<smiles>N=C(N)NCCCC1NC(=O)C(C(O)(c2c[nH]c3cc(Br)ccc23)c2c(Br)ccc3c(=O)o[nH]c23)NC1=O</smiles>

(71) bromobenzisoxazolone barettin

Figure 13. Barettin derivatives. 
Analysis of the marine-derived Streptomyces sp. isolate Mei37 led to isolation of mansouramycins A-D with mansouramycin D (72), being the only indole-derived congener (Figure 14). Mansouramycins A-C display significant cytotoxic effects [62].<smiles>CNC1=CC(=O)c2cc(-c3c[nH]c4ccccc34)ncc2C1=O</smiles>

(72) mansouramycin D

Figure 14. Mansouramycin D.

With 2-(1H-indol-3-yl)ethyl 2-hydroxypropanoate (73) and 2-(1H-indol-3-yl)ethyl 5-hydroxypentanoate (74), two new indole derivatives were isolated from a marine sponge-derived yeast strain USF-HO25, identified as Pichia membranifaciens (Figure 15). Both compounds showed weak activity as 2,2-diphenyl-1-picrylhydrazyl (DPPH) radical scavengers [63].

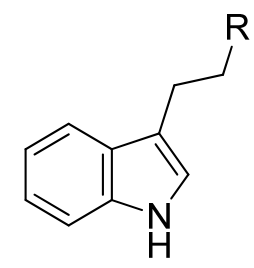

(73) 2-(1H-indol-3-yl)ethyl 2-hydroxypropanoate<smiles>CC(O)C(=O)O[R]#[W]</smiles>

(74) 2-(1H-indol-3-yl)ethyl 5-hydroxypentanoate<smiles>[R][CH][14CH2]OC(=O)CCCCCO</smiles>

Figure 15. 2-(1H-Indol-3-yl)ethyl 2-hydroxypropanoate and 2-(1H-indol-3-yl)ethyl 5-hydroxypentanoate.

1-(1H-Indol-3-yl)-2,3-dihydroxy-5-methyl-hexane (75) was isolated from the South China Sea sponge Axinella sp. (Figure 16) [64].<smiles>CC(C)CC(O)C(O)Cc1c[nH]c2ccccc12</smiles>

(75) 1-(1H-indol-3-yl)-2,3-dihydroxy-5-methyl-hexane

Figure 16. 1-(1H-Indol-3-yl)-2,3-dihydroxy-5-methyl-hexane. 
Trachycladindoles A-G (76-82), isolated from the southern Australian marine sponge Trachycladus laevispirulifer, bear a 2-amino-4,5-dihydroimidazole moiety (Figure 17). They displayed a substitution pattern-dependent cytotoxic activity against human cancer cell lines A549, HT-29 and MDA-MB-231 [65].<smiles>[R]c1cc2[nH]c(C(=O)[O-])c(C3C([R])N([R])C(=[NH2+])N3C)c2cc1[R]</smiles>
(76) $\mathrm{R}^{1}=\mathrm{Br}, \mathrm{R}^{2,3,4}=\mathrm{H}$
trachycladindole $\mathrm{A}$
(77) $\mathrm{R}^{1}=\mathrm{Br}, \mathrm{R}^{2,4}=\mathrm{H}, \mathrm{R}^{3}=\mathrm{Me}$ trachycladindole B
(78) $\mathrm{R}^{1}=\mathrm{Br}, \mathrm{R}^{2}=\mathrm{OH}, \mathrm{R}^{3,4}=\mathrm{H} \quad$ trachycladindole $\mathrm{C}$
(79) $\mathrm{R}^{1}=\mathrm{Br}, \mathrm{R}^{2}=\mathrm{OH}, \mathrm{R}^{3}=\mathrm{Me}, \mathrm{R}^{4}=\mathrm{H}$ trachycladindole $\mathrm{D}$
(80) $\mathrm{R}^{1}=\mathrm{Br}, \mathrm{R}^{2}=\mathrm{H}, \mathrm{R}^{3}=\mathrm{Me}, \mathrm{R}^{4}=\mathrm{OH}$ trachycladindole $\mathrm{E}$
(81) $\mathrm{R}^{1}=\mathrm{Br}, \mathrm{R}^{2,4}=\mathrm{OH}, \mathrm{R}^{3}=\mathrm{Me} \quad$ trachycladindole $\mathrm{F}$
(82) $R^{1,2,3,4}=H$
trachycladindole $\mathrm{G}$

Figure 17. Trachycladindoles A-G.

Aqabamycins A-G were isolated from fermentation broths of a marine bacterium, Vibrio sp., isolated from Red Sea soft coral Sinularia polydactyla, aqabamycin G (83) being the only indole derivative (Figure 18) [66,67].<smiles>O=C1NC(=O)C(c2c[nH]c3ccccc23)=C1c1ccc(O)c([N+](=O)[O-])c1</smiles>

(83) aqabamycin $\mathrm{G}$

Figure 18. Aqabamycin G.

Phidianidines A (84) and B (85) have been isolated from the marine opisthobranch mollusc Phidiana militaris and contain an uncommon 1,2,4-oxadiazole fragment (Figure 19) [68]. Both showed high cytotoxic activity towards several tumor cells [68], although other authors indicated a lack of cytoxicity [69,70]. Phidianidine A (84) was identified as a new CXCR4 (chemokine receptor) ligand and inhibits CXCL12 (C-X-C motif chemokine 12)-induced DNA synthesis, cell migration, and ERK1/2 (extracellular-signal-regulated kinases) activation [69]. Phidianidines displayed inhibitory activity on the dopamine transporter (DAT), but no activity towards the norepinephrine (NET) and the serotonin transporter (SERT). Furthermore, they represent selective and potent ligands of the $\mu$-opioid receptor with no activity on $\delta$ - or $\kappa$-opioid receptors [70]. Phidianidine analogs were tested as neuroprotective agents, showing activity against $\mathrm{A} \beta_{25-35^{-}}, \mathrm{H}_{2} \mathrm{O}_{2-}$ and OGD (oxygen-glucose deprivation)-induced neurotoxicity in SH-SY5Y cells [71]. 


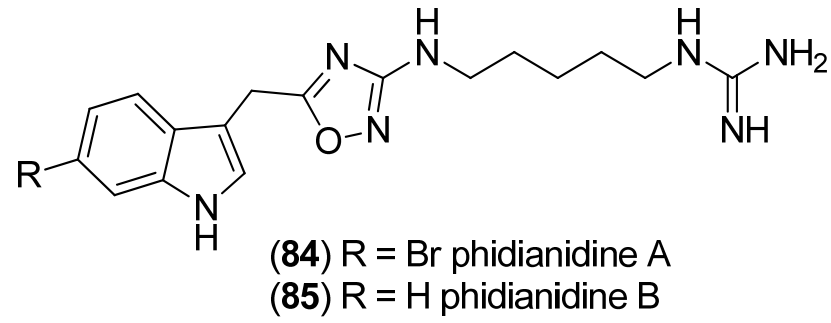

Figure 19. Phidianidines A and B.

Kororamide A (86) was obtained from the Australian bryozoan Amathia tortuosa and exists as a mixture of its cis and trans amide rotamer (Figure 20) [72].<smiles>CN(/C=C\c1ccc2c(/C=C\N(C)C(=O)[C@@]3(C)CCCN3C)c[nH]c2c1Br)C(=O)[C@H]1CCCN1C</smiles>

(86) kororamide A

Figure 20. Koroamide A, mixture of cis- and trans amide rotamer.

Analysis of the marine bacterium Pantoea agglomerans P20-14 resulted in the isolation of 3-( $p$-hydroxy)benzoyl indole (87) and $N$-(4-hydroxyphenethyl)-2-(1H-indol-3-yl)acetamide (88) (Figure 21), together with bisindole 1,2-di(1H-indol-3-yl)ethane (506, see Figure 84) [73].<smiles>O=C(c1ccc(O)cc1)c1c[nH]c2ccccc12</smiles>

(87)<smiles>O=C(Cc1c[nH]c2ccccc12)NCCc1ccc(O)cc1</smiles>

(88)

Figure 21. 3-( $p$-Hydroxy)benzoyl indole and $N$-(4-hydroxyphenethyl)-2-(1H-indol-3-yl)acetamide.

Leptoclinidamines A-C (89-91) were isolated from the Australian ascidian Leptoclinides durus (Figure 22). None of the compounds was active in antimalarial, antitrypanosomal and cytotoxic activity tests [74]. Leptoclinidamide (92) and (-)-leptoclinidamine B (93) were isolated from the Indonesian ascidian Leptoclinides dubius, together with $\mathrm{C}^{2}-\alpha$-D-mannosylpyranosyl-L-tryptophan (9). They did not exhibit antifungal, antibacterial (Gram-positive and Gram-negative) or cytotoxic (HCT-15 and Jurkat cell lines) activity [75]. 
<smiles>[R]c1ccc2c(C(=O)C(=O)N[C@@H](CCCNC(N)=[NH2+])C(=O)O)c[nH]c2c1</smiles>

(89) $\mathrm{R}=\mathrm{H}, 13 \mathrm{~S} \quad$ leptoclinidamine $\mathrm{A}$

(90) $\mathrm{R}=\mathrm{OH}, 13 \mathrm{~S}(+)$-leptoclinidamine $\mathrm{B}$

(93) $\mathrm{R}=\mathrm{OH}, 13 R$ (-)-leptoclinidamine $\mathrm{B}$<smiles>CSc1c(CC(NC(=O)c2c[nH]c3cc(Br)ccc23)C(=O)O)[n+](C)cn1C</smiles>

(91) leptoclinidamine C<smiles>NCCC(=O)NCCC(=O)NCCc1c[nH]c2ccccc12</smiles>

(92) $\mathrm{R}=\mathrm{H}, 13 \mathrm{~S}$ leptoclinidamide

Figure 22. Leptoclinidamines $\mathrm{A}-\mathrm{C}$ and leptoclimidamide.

Bunodosine 391 (BDS 391, 94) was isolated from the sea anemone Bunodosoma cangicum and showed analgetic effects mediated via serotonin receptors (Figure 23) [76].<smiles>O=C(Cc1c[nH]c2cc(Br)ccc12)N[C@@H](Cc1c[nH]cn1)C(=O)O</smiles>

(94) bunodosine 391

Figure 23. Bunodosine 391 (BDS 391).

Pyrinodemin F (95) was obtained from an Okinawan marine sponge Amphimedon sp. (Figure 24) [77].<smiles>O=C(NCCCCCCC#CCCCCCCCCCc1cccnc1)C(=O)c1c[nH]c2ccccc12</smiles>

(95) pyrinodemin F

Figure 24. Pyrinodemin F.

Cytoglobosins $A-G$ (96-102), isochaetoglobosin D (103) and chaetoglobosin $F_{e x}$ (104) were isolated from the marine green alga derived endophytic fungus Chaetomium globosum QEN-14 (Figure 25). Cytoglobosins C (98) and D (99) exhibited cytotoxic activity towards the cancer cell line A549 (IC50-values 2.26 and $2.55 \mu \mathrm{M}$ ), whereas the other cytoglobosins were inactive [78].

Nakijinamines A-I (105-113) and 6-bromoconicamin (114) have been isolated from the Okinawan marine sponge Suberites sp. (Figure 26). Nakijinamine A (105) showed antimicrobial activity against Candida albicans, Cryptococcus neoformans, Trichophyton mentagrophytes, Staphylococcus aureus, Bacillus subtilis and Micrococcus luteus. Nakijinamines C (107) and E (109) exhibited antifungal activity against Aspergillus niger, while nakijinamines B (106) and F (110) showed activity against C. albicans. None of the compounds showed cytotoxicity against mammalian cells $[79,80]$. 


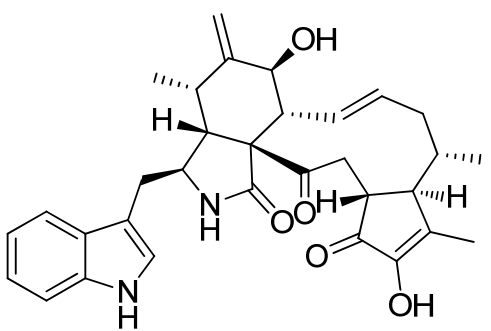

(96)

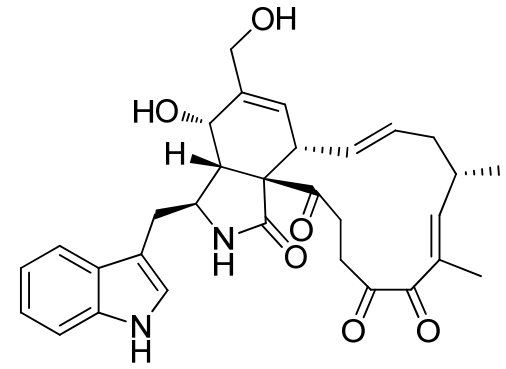

(100)

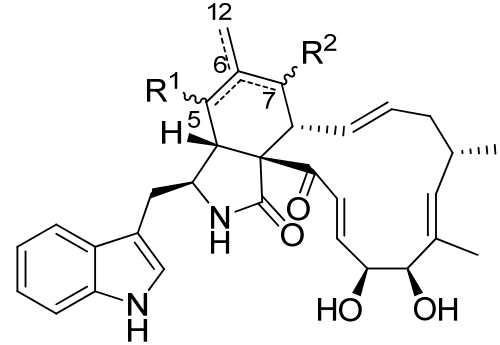

(97) $\mathrm{R}^{1}=\alpha-\mathrm{Me}, \mathrm{R}^{2}=\beta-\mathrm{OH}, \Delta^{6,12}$

(98) $\mathrm{R}^{1}=\mathrm{Me}, \mathrm{R}^{2}=\beta-\mathrm{OH}, \Delta^{5}$

(99) $\mathrm{R}^{1}=\alpha-M e, \mathrm{R}^{2}=\mathrm{H}, \Delta^{6}$

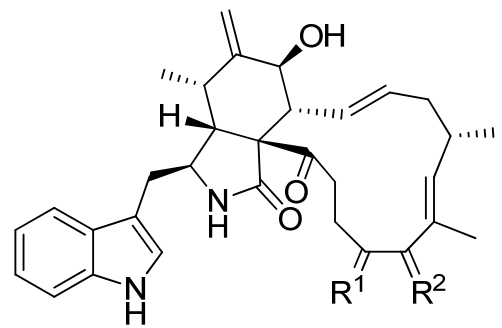

$$
\begin{aligned}
& \text { (101) } R^{1}=\mathrm{O}, \mathrm{R}^{2}=\beta-\mathrm{OH} \\
& (102) \mathrm{R}^{1,2}=\mathrm{H}, \beta-\mathrm{OH} \\
& \text { (103) } R^{1,2}=\mathrm{O} \\
& \text { (104) } \mathrm{R}^{1}=\mathrm{H}, \beta-\mathrm{OH}, \mathrm{R}^{2}=\mathrm{O}
\end{aligned}
$$

Figure 25. Cytoglobosins $A-G$, isochaetoglobosin $D$ and chaetoglobosin $F_{\text {ex. }}$.<smiles></smiles>

(105) $\mathrm{R}=$ Br nakijinamine $A$ (106) $R=H$ nakijinamine $B$<smiles></smiles>

(108) $\mathrm{R}={ }_{5} \mathrm{~s}^{\mathrm{s}} \stackrel{\ominus}{\mathrm{SO}_{3}}$ nakijinamine $\mathrm{D}$ (110) $R=-3$ (111) $R=-s^{3}$ (112) $\mathrm{R}=$<smiles></smiles>

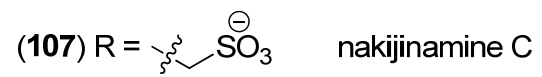<smiles></smiles><smiles>CO/C=C/c1c[nH]c2cc(Br)ccc12</smiles>

(114) 6-bromoconicamin

Figure 26. Nakijinamines A-I and 6-bromoconicamin. 
5'-[(5,6-Dibromo-1H-indol-3-yl)methyl]-3'-methylimidazolidine-2',4'-dione (115), 5,6-dibromo$1 H$-indole-3-ethyl- $N$-formylamine or 5,6-dibromo- $N$-formyltryptamine (116), 5,6-dibromo- $1 H$-indole3-ethyl- $N$-acetylamine or 5,6-dibromo- $N$-acetyltryptamine (117) and 5,6-dibromo- $N$-acetyl- $N$ methyltryptamine (118) were obtained from the Thai sponge Smenospongia sp. (Figure 27) [81].<smiles>CN1C(=O)N[C@H](Cc2c[nH]c3cc(Br)c(Br)cc23)C1=O</smiles>

(115)<smiles>[R]N([R])CCc1c[nH]c2cc(Br)c(Br)cc12</smiles>

(116) $\mathrm{R}^{1}=\mathrm{H}, \mathrm{R}^{2}=\mathrm{CHO}$

(117) $R^{1}=H, R^{2}=C O M e$

(118) $R^{1}=M e, R^{2}=$ COMe

Figure 27. 5,6-Dibromoindole derivatives.

Penilloid A (119) was isolated from the marine derived fungi Penicillium sp. and A. sydowii [82].

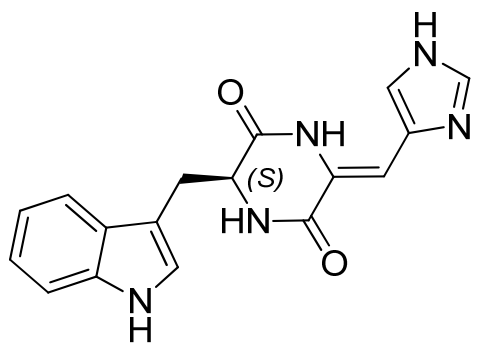

(119) penilloid $A$

Figure 28. Penilloid A.

3-Hydroxyglyantrypine (120), oxoglyantrypine (121a, 121b), cladoquinazoline (122), epi-cladoquinazoline (123) and norquinadoline A (124) were isolated from the mangrove-derived fungus Cladosporium sp. PJX-41 and exhibited antiviral activities against influenza A virus $\left(\mathrm{H}_{1} \mathrm{~N}_{1}\right)$ (Figure 29) [83].

Herdmanines A-D were isolated from the marine ascidian Herdmania momus, herdmanines A, B and D (125-127) being tryptophan-derived (Figure 30). Herdmanines C and D (127) showed anti-inflammatory activities, due to the inhibitory effect on mRNA expression of iNOS (inducible nitric oxide synthase) as well as COX-2 (cyclooxygenase 2) and IL-6 (interleukin 6) [84]. Herdmanines E-L were isolated from the same organism and displayed peroxisome proliferator-activated receptor (PPAR)- $\gamma$ agonistic activity. Herdmanines E and I-K (128-131) are tryptophan-derived [85]. Herdmanines A, B, E, I and $\mathrm{K}$ are derived from D-amino acids.

The ascidian Herdmania momus was also a source of nucleosides (132-135), compound 132 was given the trivial name momusine A (Figure 31). None of the compounds exhibited antiviral activity against a series of human pathogenic viruses [86]. 
<smiles>O=C1N[C@@H](O)c2nc3ccccc3c(=O)n2C1Cc1c[nH]c2ccccc12</smiles>

(120) 3-hydroxyglyantrypine<smiles>CC(C)[C@H]1NC(=O)[C@H](CC2(O)C(=O)Nc3ccccc32)n2c1nc1ccccc1c2=O</smiles>

(122) $3 R$ cladoquinazoline (123) 3 S epi-cladoquinazoline<smiles></smiles>

(121a) 14R 3-oxoglyantrypine

(121b) 14S 3-oxoglyantrypine<smiles>CC(C)=C1NC(=O)[C@H](CC[C@@]2(O)c3ccccc3N3C(=O)[C@H](C)N[C@@H]32)C2=C(C)C(=O)N1c1ccccc1N2</smiles>

(124) norquinadoline $A$

Figure 29. Glyantrypine derivatives, cladoquinazoline, epi-cladoquinazoline and norquinadoline A.<smiles>[R]c1cc2[nH]cc(C(=O)Oc3ccc(C(=O)N[C@H](CCCNC(=N)N)C(=O)O)cc3)c2cc1[R]</smiles>

(125) $\mathrm{R}^{1,2}=\mathrm{H} \quad$ herdmanine $A$

(126) $R^{1}=H, R^{2}=O H$ herdmanine $B$

(128) $R^{1}=\mathrm{OH}, \mathrm{R}^{2}=\mathrm{Br}$ herdmanine $\mathrm{E}$<smiles>[R]C(=O)C(=O)c1c[nH]c2cc(O)ccc12</smiles>

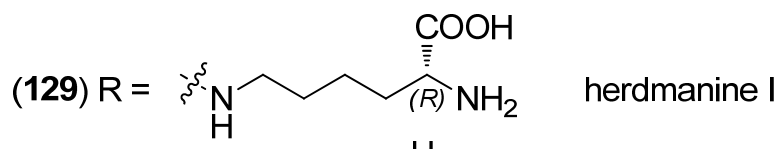
herdmanine $\mathrm{K}$<smiles>N[C](Cc1ccc(OC(=O)c2c[nH]c3cc(Br)c(O)cc23)cc1)C(=O)O</smiles>

(127) herdmanine D<smiles>Cn1cc(C(=O)NCCCC[C@H](N)C(=O)O)c2ccccc21</smiles>

(130) herdmanine J

Figure 30. Herdmanines A-K. 


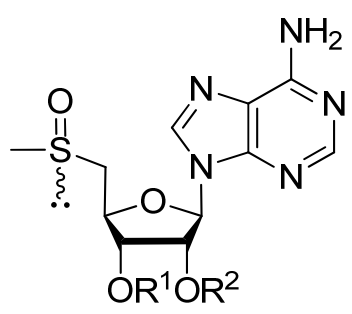

(132) $R^{1}=X, R^{2}=H$ momusine $A$

(133) $R^{1}=H, R^{2}=X$

(134) $R^{1}=X, R^{2}=H$ (epimer of $X$ at sulfur atom)

(135) $R^{1}=H, R^{2}=X$ (epimer of $X$ at sulfur atom)<smiles>[Y]=C(C)c1c[nH]c2cc(Br)c(O)cc12</smiles>

Figure 31. Momusines.

Didemnidines A (136) and B (137), two indole spermidine alkaloids, were isolated from the New Zealand ascidian Didemnum sp. (Figure 32). Both were found to be inactive as phospholipase $\mathrm{A}_{2}$ and farnesyltransferase enzyme inhibitors and not cytotoxic, but didemnidine B (137) showed mild antiparasitic activity against the malaria parasite Plasmodium falciparum [87].

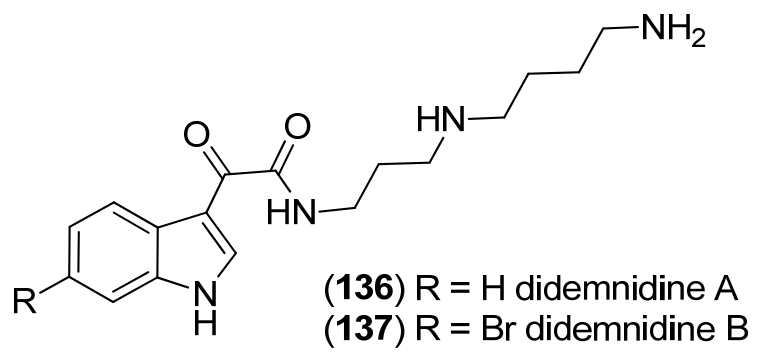

Figure 32. Didemnidines A and B.

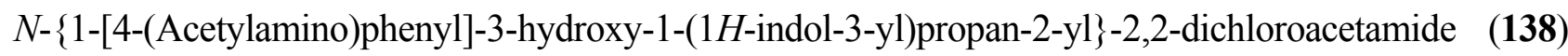
was isolated from a deep-sea sediment metagenomic clone-derived Escherichia coli fermentation broth and found to have analgetic activity (Figure 33) [88]. It has a remarkable structural resemblance to the antibiotic chloramphenicol.<smiles>CC(=O)Nc1ccc(C(c2c[nH]c3ccccc23)C(CO)NC(=O)C(Cl)Cl)cc1</smiles>

(138)

Figure 33. $\quad N$-\{1-[4-(Acetylamino)phenyl]-3-hydroxy-1-(1H-indol-3-yl)propan-2-yl $\}-2,2-$ dichloroacetamide.

Fermentation of deep-sea bacterium Shewanella piezotolerans WP3 yielded three new indole alkaloids namely shewanellines A-C. Shewanellines A (507) and B (508) belong to the bisindole alkaloids (see Figure 85). Shewanelline C (139) displayed cytotoxic activity against the tumor cell lines HL-60 and BEL-7402 (IC50 5.91 and $10.03 \mu \mathrm{g} / \mathrm{mL}$, respectively) (Figure 34) [89]. 


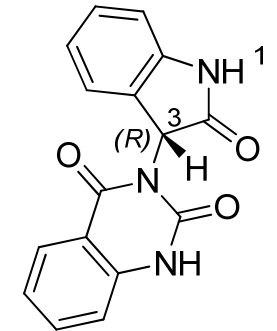

(139) shewanelline C

Figure 34. Shewanelline C.

Tanjungides A (140) ( $Z$ isomer) and B (141) ( $E$ isomer), two dibrominated indole enamides, have been isolated from the tunicate Diazona cf. Formosa and were found to have significant cytotoxicity against human tumor cell lines (Figure 35). In the same publication, the first total synthesis of these compounds is reported employing methyl $1 H$-indole-3-carboxylate as starting material [90].

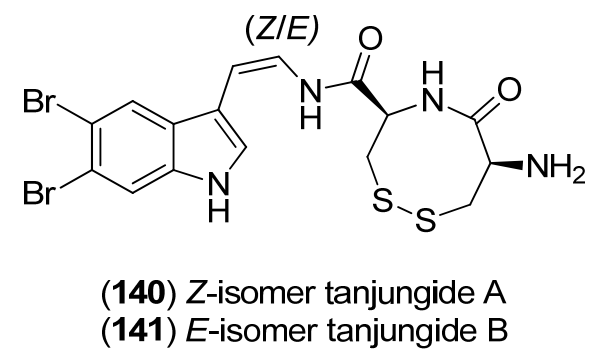

Figure 35. Tanjungides A and B.

Examination of actinomycete Actinomadura BCC 24717 led to the isolation of 1-hydroxymethylindole-3-carboxylic acid (142) and 1-methyl indole-3-carboxamide (143) with the latter compound displaying antifungal activity against Candida albicans ( $\mathrm{IC}_{50} 41.97 \mu \mathrm{g} / \mathrm{mL}$ ) (Figure 36) [91].

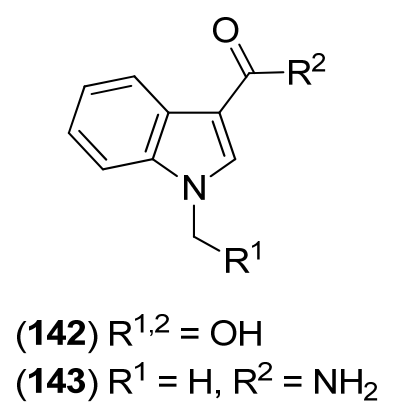

Figure 36. 1-Hydroxymethylindole-3-carboxylic acid and 1-methyl indole-3-carboxamide.

Streptomycindole (144) was isolated from Streptomyces sp. DA22, associated with the South China Sea sponge Craniella australiensis, and was found to be inactive against the tumor cell lines HL-60, HCT-116, HO-8910 and HepG2 (Figure 37) [92]. 


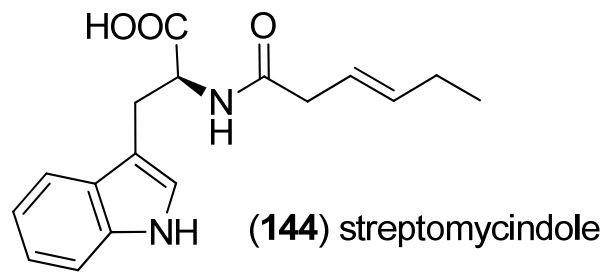

Figure 37. Streptomycindole.

Almazolone (145) was isolated from the red alga Haraldiophyllum sp., collected in Dakar (Senegal) as an 88:12 mixture of $(Z) /(E)$ stereoisomers (Figure 38). Photoisomerization of the $(Z)$ into the $(E)$-isomer, as well as slow thermal reisomerization of the $(E)$-isomer, was observed [93].

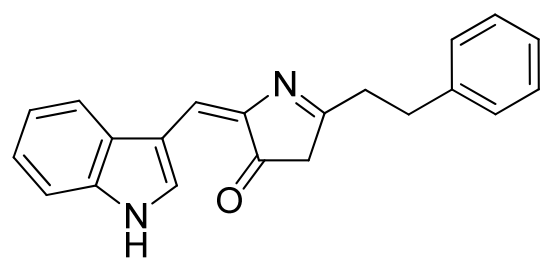

(145) (E)-almazolone<smiles>O=C1CC(CCc2ccccc2)=N/C1=C\c1c[nH]c2ccccc12</smiles>

(145) (Z)-almazolone

Figure 38. Almazolone.

Iotrochamides A and B were isolated from the Australian sponge Iotrochota sp., iotrochamide B (146) bearing an indole moiety (Figure 39). Both compounds show inhibitory effects against the human pathogenic protozoon Trypanosoma brucei brucei ( $\mathrm{IC}_{50} 3.4$ and $4.7 \mu \mathrm{M}$, respectively) [94].<smiles>COC(=Cc1ccccc1)C(=O)N[C@@H](Cc1c[nH]c2cc(Br)ccc12)C(=O)O</smiles>

(146) iotrochamide B

Figure 39. Iotrochamide $B$.

3-((6-Methylpyrazin-2-yl)methyl)-1H-indole (147) was isolated from the deep-sea actinomycete Serinicoccus profundi sp. nov. and exhibited weak antimicrobial activity against Staphylococcus aureus ATCC 25923 but no cytotoxic effects against BEL7402 and HL-7702 cell lines (Figure 40) [95].<smiles>Cc1cncc(Cc2c[nH]c3ccccc23)n1</smiles>

(147) 3-((6-methylpyrazin-2-yl)methyl)-1H-indole

Figure 40. 3-((6-Methylpyrazin-2-yl)methyl)-1H-indole. 
Breitfussins A (148) and B (149) were isolated from the Arctic hydrozoan Thuiaria breitfussi (Figure 41) [96].<smiles>COc1cc(Br)cc2[nH]cc(-c3oc(-c4ccc[nH]4)nc3I)c12</smiles>

(148) breitfussin A<smiles>COc1cc(Br)cc2[nH]cc(-c3cnc(-c4ccc(Br)[nH]4)o3)c12</smiles>

(149) breitfussin B

Figure 41. Breitfussins A and B.

5-Hydroxyindole alkaloids 5-hydroxyindole-3-glyoxylate methyl ester (150) and (151), together with the bisindole scalaridine A (505, see Figure 83), were isolated from the marine sponge Scalarispongia sp. collected near Dokdo island (Figure 42). Since 151 was the monoindole analog of hyrtinadine A (496, see Figure 80), it was named hyrtinadine B [97]. Hainanerectamines A-C (152, 153 and $\mathbf{X}$ ) have been isolated from the Hainan marine sponge Hyrtios erectus, hainanerectamine $\mathrm{C}$ (800) belonging to the group of $\beta$-carboline alkaloids (see Figure 147). Hainanerectamines B (153) and $\mathrm{C}(\mathbf{8 0 0})$ display moderate inhibitory effects on the serine/threonine kinase Aurora A (IC50 24.5 and $18.6 \mu \mathrm{g} / \mathrm{mL}$ ), which is involved in cell division regulation, but none of the compounds had cytotoxic effects on the tumor cell lines A549 and HT-29 [98].<smiles>COC(=O)C(=O)c1c[nH]c2ccc(O)cc12</smiles>

(150)<smiles>Oc1ccc2[nH]cc(-c3cncnc3)c2c1</smiles>

(151)<smiles>[R]c1c[nH]c2ccc(O)cc12</smiles>

(152) R<smiles>[R]=[V]</smiles><smiles>CC(C)=O</smiles><smiles>CCCC</smiles>
hainanerectamine $\mathrm{A}$ (153) $\mathrm{R}=$<smiles>CC=C(C)CO</smiles>
hainanerectamine $\mathrm{B}$

Figure 42. 5-Hydroxyindole-3-glyoxylate methyl ester, hyrtinadine B and Hainanerectamines.

\subsection{Prenylated Indoles}

Prenylated indole alkaloids represent a large subgroup of the indole alkaloids and provide various potent biological activities. Their wide distribution in terrestrial and marine organisms nicely reflects the high nucleophilicity of the indole core which is an adequate match for the electrophilic reactivity of prenyl-type electrophiles generated from the corresponding pyrophosphates [99]. Biosynthetically, tryptophan is the indole source in most cases [100]. 
(Indole- $N$-isoprenyl)-tryptophan-valine diketopiperazine (154) was isolated from the M-3 strain belonging to the Ascomycota phylum and was shown to have a strong and selective antifungal activity against Pyricularia oryzae (Figure 43) [101]. Cyclomarazines A (155) and B (156) were isolated from the marine bacterium Salinispora arenicola CNS-205 and exhibited antimicrobial activities [102]. 5-Dimethylallylindole-3-carboxylic acid (157) was obtained from the marine-derived Streptomyces sp. MS239 and did not show antibacterial activity [103]. Dipodazine derivative (158) was isolated from the mangrove-derived endophytic fungus Penicillium chrysogenum MTCC 5108 and showed antibacterial activity [104]. Brocaeloids A-C were obtained from cultures of Penicillium brocae MA-192, an endophytic fungus isolated from the fresh leaves of the marine mangrove plant Avicennia marina. They contain a C-2 reversed prenylation, but only brocaeloid C (159) is tryptophan-derived. It showed weak or no antibacterial or DPPH radical scavenging activity and no lethality against brine shrimp (Artemia salina) [105]. Penipalines A-C (160-162) were isolated from the deep-sea-sediment derived fungus Penicillium paneum SD-44. Penipalines B (161) and C (162) showed cytotoxic effects against the A549 (IC50 20.44 and $21.54 \mu \mathrm{M}$ ) and HCT-116 (IC50 14.88 and $18.54 \mu \mathrm{M}$ ) tumor cell lines [106]. 3-((1-Hydroxy-3-(2-methylbut-3-en-2-yl)-2-oxoindolin-3-yl)methyl)-1-methyl-3,4-dihydrobenzo[e][1,4]diazepine-2,5-dione (163) was obtained from the Mediterranean sponge-derived fungus Aspergillus sp. [107].

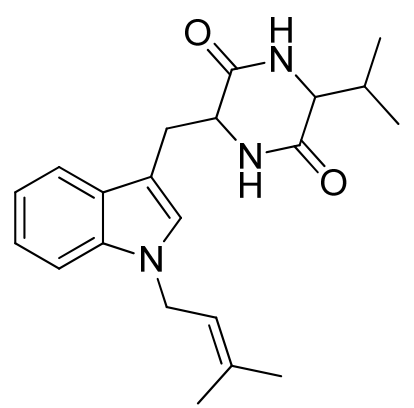

(154)

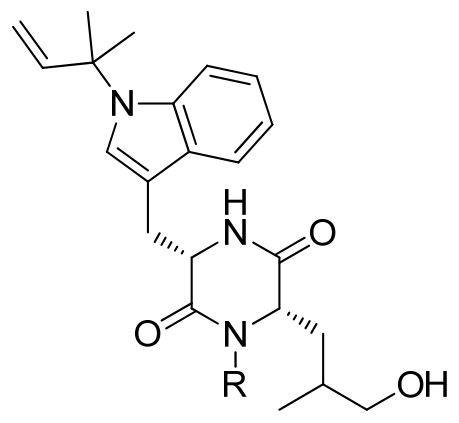

(155) $R=$ Me cyclomarazine $A$ (156) $\mathrm{R}=\mathrm{H}$ cyclomarazine $B$

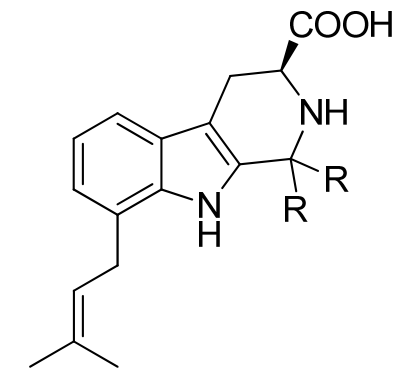

(160) $R=H$ penipaline $A$ (161) $R=$ Me penipaline $B$<smiles>CC(C)=CCc1ccc2[nH]cc(C(=O)O)c2c1</smiles>

(157)

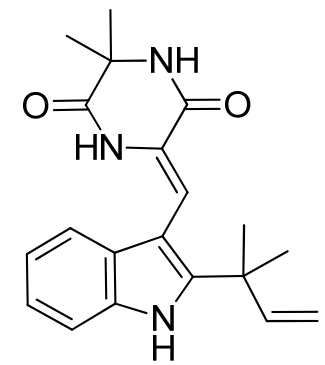

(158)

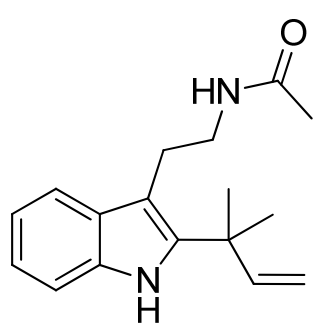

(159) brocaeloid C<smiles>C/C(=C\Cc1cccc2c(C=O)c[nH]c12)CO</smiles>

(162) penipaline $C$<smiles>C=CC(C)(C)[C@]1(CC2(C(C)(C)C)NC(=O)c3ccccc3N2C)C(=O)N(O)c2ccccc21</smiles>

(163)

Figure 43. Simple prenylated indole alkaloids.

Beginning in 1979, a group of brominated and prenylated indole alkaloids, named flustramines, have been isolated from the marine bryozoan Flustra foliacea [108-110], some of them having cytotoxic and antimicrobial [111], muscle relaxant [112], as well as butyrylcholinesterase (BChE) 
inhibitory effects [113]. From the same organism, several new alkaloids have been isolated, namely 6-bromo-2-(1,1-dimethyl-2-propenyl)-1H-indole-3-carbaldehyde (164), $\mathrm{N}$-(2-[6-bromo-2-(1,1-dimethyl-2propenyl)-1 $H$-indol-3-yl]ethyl)- $N$-methyl-methanesulfonamide (165), deformylflustrabromine (166), $\left(3 \mathrm{a} R^{\prime}, 8 \mathrm{a} R^{\prime}\right)$-6-bromo-3a-[(2E)-3,7-dimethyl-2,6-octadienyl]-1,2,3,3a,8,8a-hexahydropyrrolo[2,3-b]indol-7-ol (167) and deformylflustrabromine B (168) (Figure 44) [114,115]. Deformylflustrabromine (166) and several synthetic derivatives were found to have inhibitory effects on bacterial indole signaling and, therefore, inhibit biofilm formation in Escherichia coli and Staphylococcus aureus [116] Deformylflustrabromine (166) and deformylflustrabromine B (168) show high and subtype selective affinity to nicotinic acetylcholine (nACh) receptors [114,117]. Flustramines F-P (169-179) have recently been isolated from $F$. foliacea, flustramines $\mathrm{O}(\mathbf{1 7 8})$ and $\mathrm{P}(\mathbf{1 7 9})$ being dimers, which may possibly be isolation artifacts [118].<smiles>C=CC(C)(C)c1[nH]c2cc(Br)ccc2c1C=O</smiles>

(164)

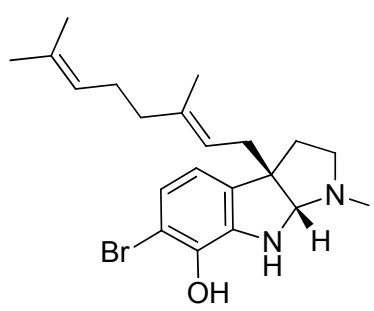

(167)

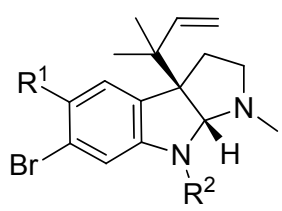

(169) $R^{1}=H, R^{2}=$ Ac flustramine $F$ (170) $R^{1}=B r, R^{2}=H$ flustramine $G$

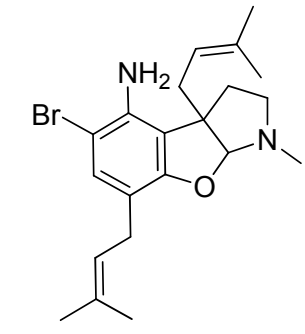

(177) $\mathrm{R}=\mathrm{H}$ flustramine $\mathrm{N}$

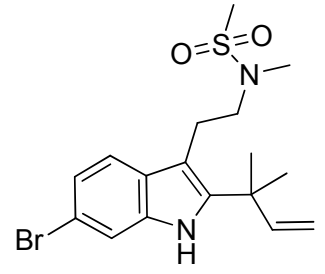

(165)

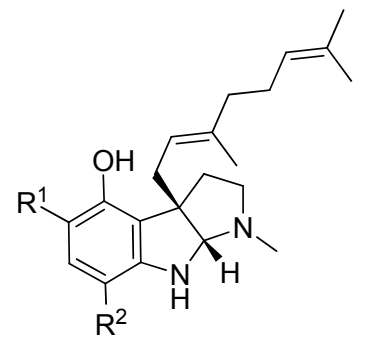

(171) $\mathrm{R}^{1}=\mathrm{H}, \mathrm{R}^{2}=\mathrm{Br}$ flustramine $\mathrm{H}$ (173) $\mathrm{R}^{1,2}=\mathrm{Br}$ flustramine $\mathrm{J}$

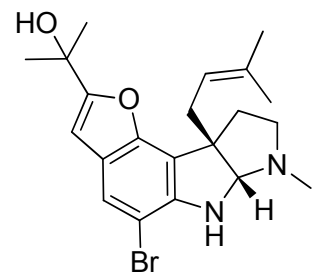

(176) $R=H$ flustramine $M$
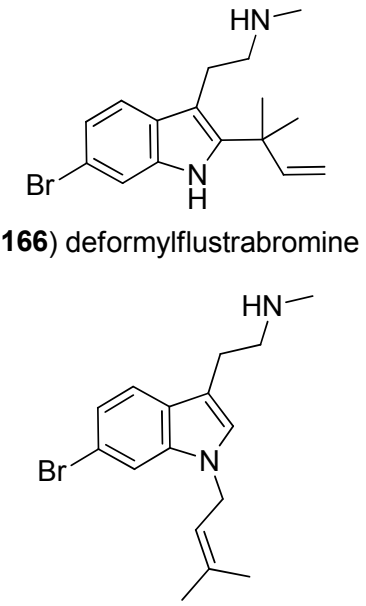

(168) deformylflustrabromine B<smiles>[R]c1cc(Br)c2c(c1O)[C@@]1(CC=C(C)C1)N(C)CN2C</smiles>

(172) $\mathrm{R}=\mathrm{H}$ flustramine I (174) $\mathrm{R}=\mathrm{Br}$ flustramine $\mathrm{K}$ (175) flustramine $L$<smiles>[R][14CH2][14CH2]C=C(C)C</smiles><smiles>C=CC(C)(C)C12CCN(C)[C@@H]1N(CN1c3cc(Br)ccc3[C@@]3(C(C)(C)C=C)CCN(C)[C@@H]13)c1cc(Br)ccc12</smiles>

(178) flustramine $O$

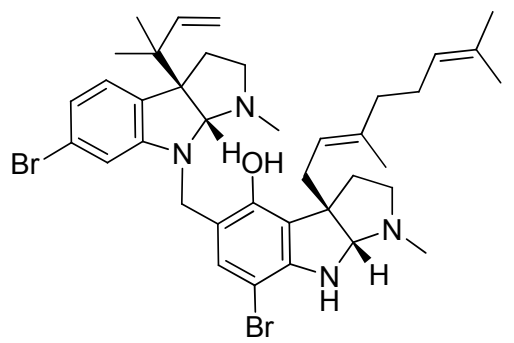

(179) flustramine $P$

Figure 44. Prenylated indole alkaloids from Flustra foliacea. 
Notoamides are a class of prenylated indole alkaloids derived from proline, tryptophan and one or two isoprene units. They are closely related to brevianamides, paraherquamides, marcfortines, asperparalines and stephacidins (Figure 45) [119,120], which show cytotoxic [121], insecticidal [122], antibiotic and antiparasitic [123,124] activities. Notoamides A-D (180-183) were isolated from a mussel-derived Aspergillus species (Figure 46). Notoamides A-C showed moderate cytotoxicity against $\mathrm{HeLa}$ and $\mathrm{L} 1210$ cell lines ( $\mathrm{IC}_{50} 22-52 \mu \mathrm{g} / \mathrm{mL}$ ) and notoamide C induces G2/M-cell cycle arrest at $6.3 \mu \mathrm{g} / \mathrm{mL}$ (cell line not specified) [125]. Notoamide E (184) was identified to be a short-lived precursor in the biosynthesis of prenylated indole alkaloids in Aspergillus sp. and a feeding experiment of ${ }^{13} \mathrm{C}$-labeled notoamide $\mathrm{E}$ afforded structurally novel metabolites [126]. Notoamides $\mathrm{F}-\mathrm{R}$ (185-197) [127-129], 17-epi-notoamides M (198) and Q (199) [130] and (-)-versicolamide B (203, Figure 47) [128] have been isolated from Aspergillus sp. Noteworthy are notoamide O (194) with its unprecedented hemiacetal/hemiaminal ether structure and notoamide P (195) as the first brominated member of the notoamides family. The absolute configuration of notoamide $\mathrm{C}$ (182) was first reported as $3 R$, but later revised to as $3 S$ on the basis of X-ray crystallography and CD spectroscopy $[130,131]$. Thereafter, the configuration of notoamides Q (196) and M (192) was also corrected to $3 S$ [132] as was the configuration of notoamide B (181) [130]. The absolute configuration of notoamide $\mathrm{J}$ was revised to be $3 R$ [133]. Iso-notoamide B (200) was isolated from the marine-derived endophytic fungus Paecilomyces variotii EN-291 [134]. Notoamide C (182) displayed significant anti-fouling activity and strong antilarval settlement activity against Bugula neritina [135]. Notoamide I (188) was found to show weak cytotoxicity against HeLa cells ( $\mathrm{IC}_{50} 21 \mu \mathrm{g} / \mathrm{mL}$ ) [127]. Recently, notoamide S (201), which has been assumed to be a key intermediate in the biosynthesis of stephacidin and notoamide families, has been isolated from Aspergillus amoenus [120,136]. Notoamide T (202) was furthermore identified to be a biosynthetic precursor of stephacidin $\mathrm{A}$ and notoamide $\mathrm{B}$, as proven by ${ }^{13} \mathrm{C}$ feeding experiments [20,137]. Biosynthetic pathways of the notoamide, paraherquamide and malbrancheamide family are discussed by Li et al. [138].

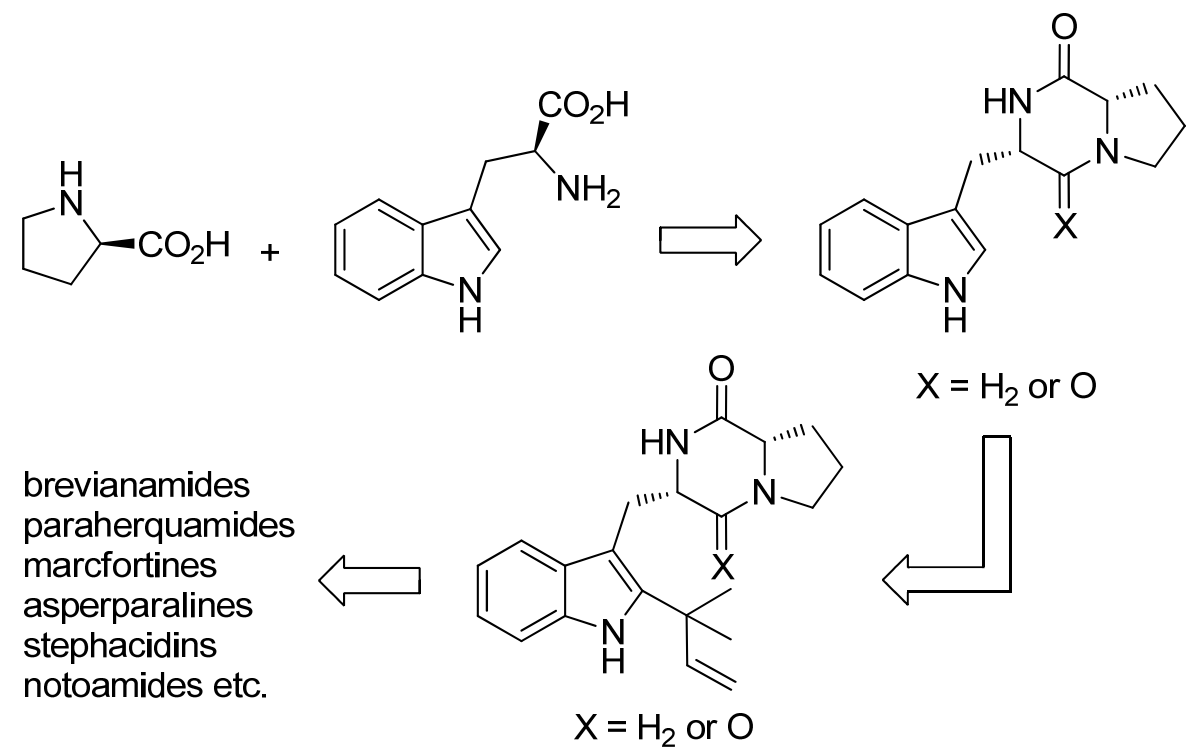

Figure 45. Biosynthetic pathway to brevianamides, paraherquamides, marcfortines, asperparalines, stephacidins and notoamides. 


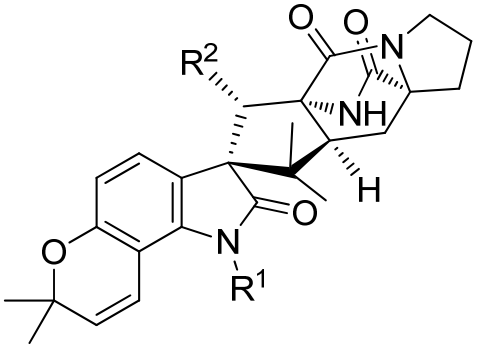

(180) $\mathrm{R}^{1}=\mathrm{OH}, \mathrm{R}^{2}=\mathrm{H}$ notoamide $\mathrm{A}$ (187) $\mathrm{R}^{1,2}=\mathrm{OH}$ notoamide $\mathrm{H}$ (193) $R^{1,2}=H$ notoamide $N$

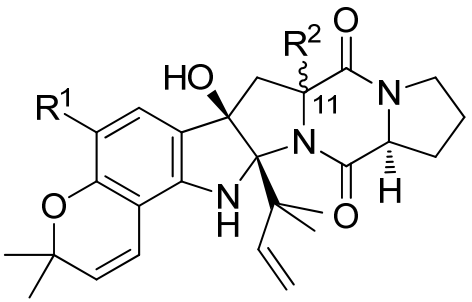

(183) $R^{1,2}=H, 11 S$ notoamide $D$ (190) $R^{1}=\mathrm{H}, \mathrm{R}^{2}=\mathrm{OH}, 11$ S notoamide $\mathrm{K}$ (195) $\mathrm{R}^{1}=\mathrm{Br}, \mathrm{R}^{2}=\mathrm{OH}, 11 S$ notoamide $\mathrm{P}$<smiles>C=CC(C)(C)C1(C[C@H]2NC(=O)[C@@H]3CCCN3C2=O)C(=O)Nc2cc(O)ccc21</smiles>

(189) notoamide J

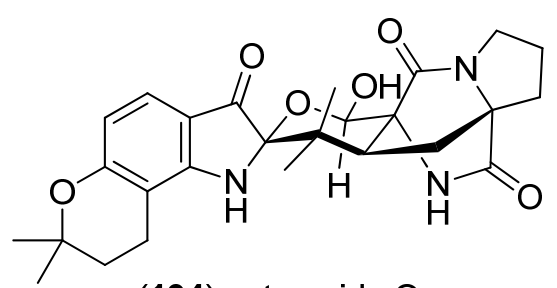

(194) notoamide $\mathrm{O}$

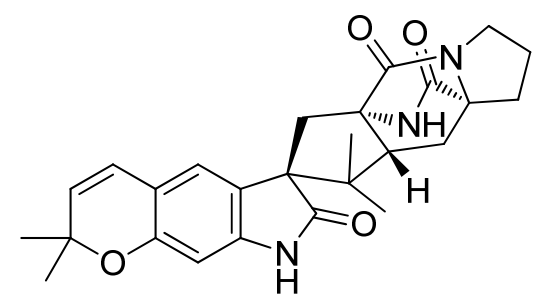

(200) iso-notoamide B<smiles>C=CC(C)(C)[C@]1(C[C@@H]2NC(=O)[C@@H]3CCCN3C2=O)C(=O)Nc2c1ccc1c2C=CC(C)(C)O1</smiles>

(182) notoamide C<smiles>C=CC(C)(C)c1[nH]c2c3c(ccc2c1C[C@@H]1NC(=O)[C@@H]2CCCN2C1=O)C=CC(C)(C)O3</smiles>

(184) notoamide E

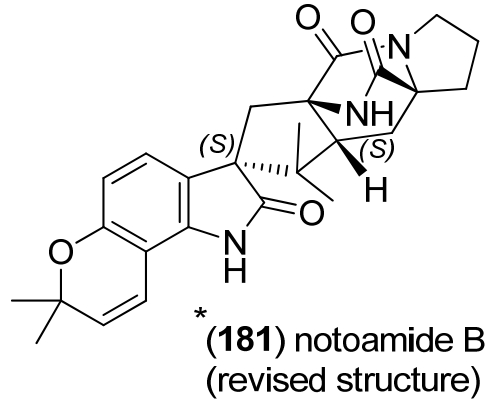

(185) $R^{1,2}=H, R^{3}=$ OMe notoamide $F$ (186) $R^{1}=\mathrm{OH}, \mathrm{R}^{2}=\mathrm{H}, \mathrm{R}^{3}=$ OMe notoamide $\mathrm{G}$ (188) $R^{1}=H, R^{2,3}=$ O notoamide I (197) $R^{1,2}=H, R^{3}=O H$ notoamide $R$

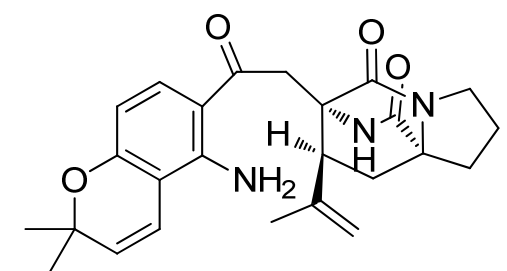

(191) notoamide L<smiles>[R]C[C@]12CCCN1C(=O)[C@@H](C[C@@H](C)C1(C(C)(C)C=C)C(=O)Nc3c1ccc1c3C=CC(C)(C)O1)NC2=O</smiles>

(192) $\mathrm{R}=\mathrm{OH}, 17 \mathrm{R}$ notoamide $\mathrm{M}$ (196) $R=$ OMe, $17 R$ notoamide $Q$ (198) $\mathrm{R}=\mathrm{OH}, 17 \mathrm{~S}$ 17-epi-notoamide $\mathrm{M}$ (199) $R=$ OMe, $17 S$ 17-epi-notoamide $Q$<smiles>C=CC(C)(C)c1[nH]c2c(CC=C(C)C)c(O)ccc2c1C[C@@H]1NC(=O)[C@H]2CCCN2C1=O</smiles>

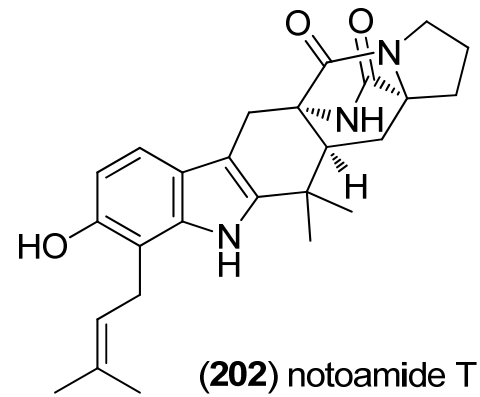

Figure 46. Notoamides A-T and iso-notoamide B.

6-epi-Stephacidin A (204), $N$-hydroxy-6-epi-stephacidin A (205) and 6-epi-avrainvillamide (206) were isolated from Aspergillus taichungensis (Figure 47). $N$-Hydroxy-6-epi-stephacidin A (205) and 6-epi-avrainvillamide (206) showed significant cytotoxic activities against HL-60 (IC50 4.45 and $1.88 \mu \mathrm{M}$ ) and A549 (IC50 3.02 and $1.92 \mu \mathrm{M}$ ) cell lines [139]. 


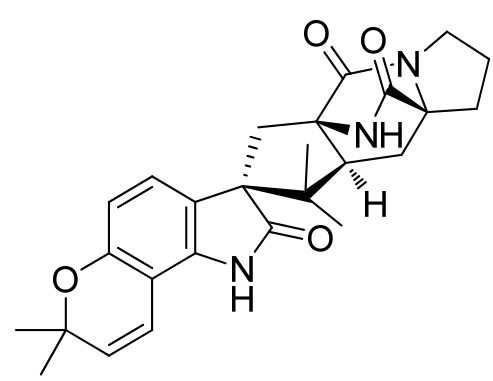

(203) (-)-versicolamide B

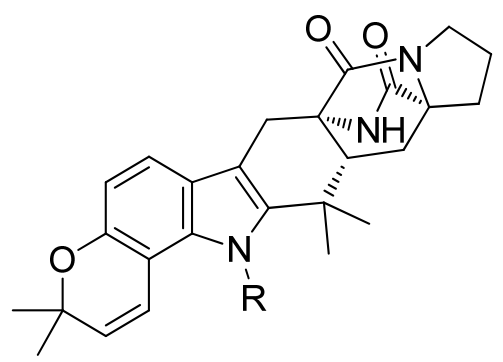

(204) R = H 6-epi-stephacidin A (205) $\mathrm{R}=\mathrm{OH}$

$N$-hydroxy-6-epi-stephacidin A

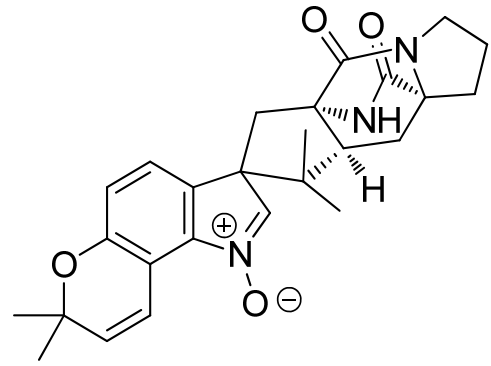

(206) 6-epi-avrainvillamide

Figure 47. (-)-Versicolamide B, 6-epi-stephacidin A, $N$-hydroxy-6-epi-stephacidin A and 6-epi-avrainvillamide.

(-)-Spiromalbramide (207) and (+)-isomalbrancheamide B (208) were identified from a marine invertebrate-derived Malbranchea graminicola strain, using direct analysis in real time (DART) mass spectrometry (Figure 48). Furthermore, (+)-malbrancheamide C (209) and (+)-isomalbrancheamide C (210) were isolated after enriching the growth medium with bromide salts [140]. Their structures resemble those of the versciolamides, the sclerotiamides and certain notoamides but the benzoid part of the indole unit is not prenylated.

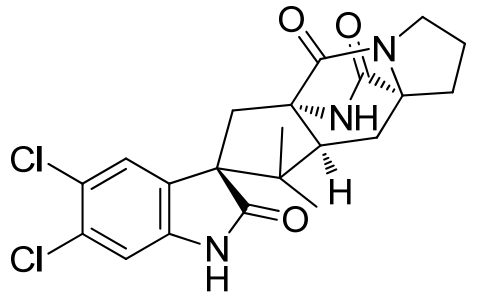

(207) (-)-spiromalbramide

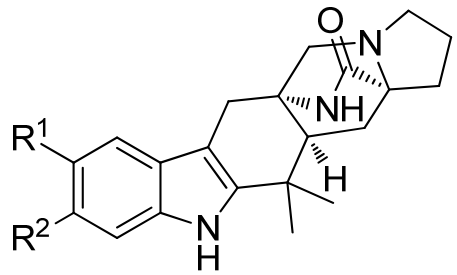

(208) $\mathrm{R}^{1}=\mathrm{Cl}, \mathrm{R}^{2}=\mathrm{H}(+)$-isomalbrancheamide $\mathrm{B}$ (209) $\mathrm{R}^{1}=\mathrm{H}, \mathrm{R}^{2}=\mathrm{Br}(+)$-malbrancheamide $\mathrm{C}$

(210) $\mathrm{R}^{1}=\mathrm{Br}, \mathrm{R}^{2}=\mathrm{H}(+)$-isomalbrancheamide $\mathrm{C}$

Figure 48. (-)-Spiromalbramide and malbrancheamides.

5-Chlorosclerotiamide (211) and 10-epi-sclerotiamide (212), together with sclerotiamide (213) and notoamide C (182) and I (188), were isolated from the deep-sea-derived fungus Aspergillus westerdijkiae DFFSCS013 (Figure 49). None of them showed cytotoxic effects against A549, HL-60, K562, and MCF-7 cell lines [141]. 5-Chlorosclerotiamide (211), together with brevianamide F, circumdatin $\mathrm{F}$ and $\mathrm{L}$ and notoamide $\mathrm{C}(\mathbf{1 8 2})$ were found to have antifouling potential and displayed potent activity against larval settlement of Bugula neritina [135]. Mangrovamides A-C (214-216) have been isolated from Penicillium sp. isolated from a mangrove sediment sample of the South China Sea. They bear a $\gamma$-methyl proline derived skeleton, unprecedented among the paraherquamide family. Mangrovamides did not show cytotoxicity against the human tumor cell lines H1975, U937, K562, BGC823, MOLT-4, MCF-7, A549, HeLa, HL-60 and Huh-7, while mangrovamide C (216) displayed moderate inhibitory effect on AChE ( $\left.\mathrm{IC}_{50} 58.0 \mu \mathrm{M}\right)$ [142]. 


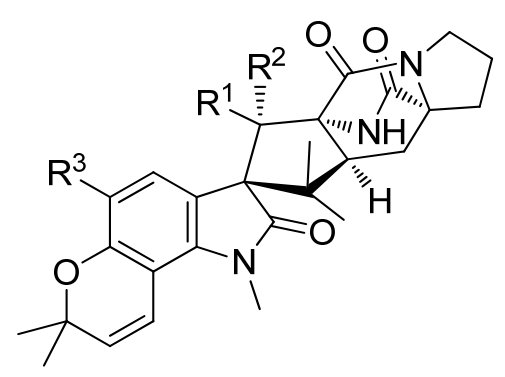

(211) $\mathrm{R}^{1}=\mathrm{H}, \mathrm{R}^{2}=\mathrm{OH}, \mathrm{R}^{3}=\mathrm{Cl}$ 5-chlorosclerotiamide (212) $\mathrm{R}^{1}=\mathrm{OH}, \mathrm{R}^{2,3}=\mathrm{H}$ 10-epi-sclerotiamide

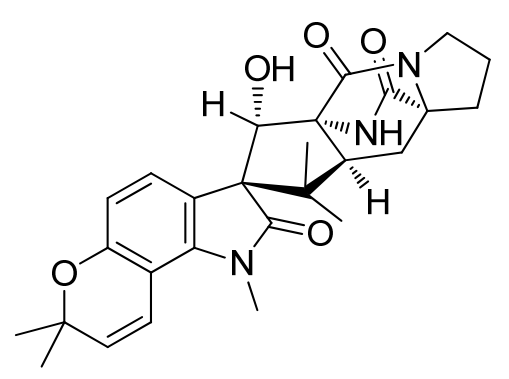

(213) sclerotiamide

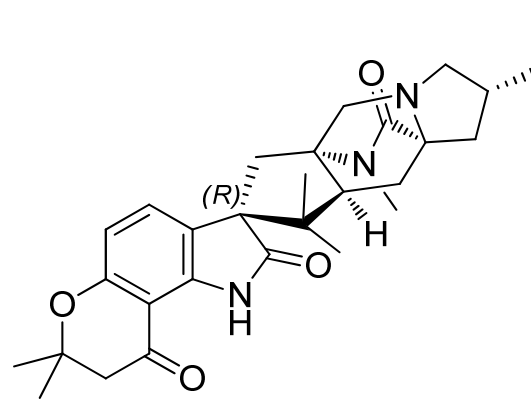

(214) mangrovamide $A$

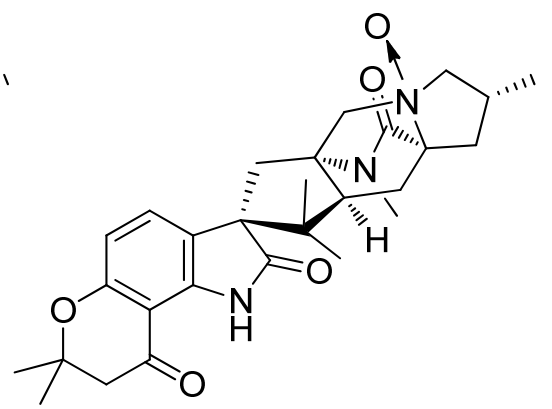

(215) mangrovamide $B$

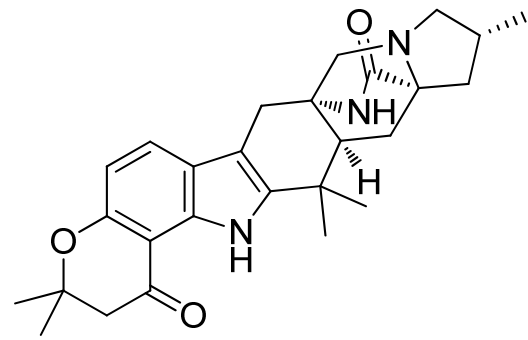

(216) mangrovamide C

Figure 49. Sclerotiamides and mangrovamides A-C.

With compound 217, spirotryprostatins C-E (218-220), two fumitremorgin B derivatives (221, 222) and 13-oxoverruculogen (223), new prenylated indole diketopiperazine alkaloids have been isolated from the holothurian-derived fungus Aspergillus fumigatus (Figure 50). All substances were tested for cytotoxic activity against MOLT-4, A549, HL-60, and BEL-7420 cell lines and showed variable activity with spirotryprostatin $\mathrm{E}$ and fumitremorgin $\mathrm{B}$ derivatives being most active [143]. Spirotryprostatin F (224) was also isolated from A. fumigatus [144]. Aspergillus sydowi PFW1-13 was the source of 6-methoxyspirotryprostatin B (225), 18-oxotryprostatin A (226) and 14-hydroxyterezine D (227). They show weak cytotoxic activity towards A549 ( $\mathrm{IC}_{50}$-values 8.29, 1.28 and $7.31 \mu \mathrm{M}$, respectively) and 14-hydroxyterezine D (227) was also active against HL-60 cells (IC50 9.71 $\mu \mathrm{M})$ [145]. Cyclotryprostatin E (228) was obtained from the marine fungal strain Aspergillus sydowii SCSIO 00305, isolated from a healthy tissue of Verrucella umbraculum [146].

Prenylcyclotryprostatin B (229), 20-hydroxycyclotryprostatin B (230), 9-hydroxyfumitremorgin C (231) and 6-hydroxytryprostatin B (232) were obtained from A. fumigatus YK-7 (Figure 51). Prenylcyclotryprostatin B (229) and 9-hydroxyfumitremorgin C (231) displayed cytotoxic activities towards U937 cell lines [147]. 
<smiles>COc1ccc2c(c1)N[C@@]1(C(=O)c3ccc(OC)cc3[C@H]1O)[C@@H](C=C(C)C)N2C(=O)[C@H]1CCCN1C</smiles>

(217)

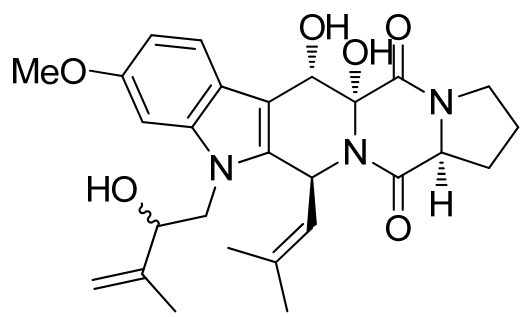

(221, 222)

fumitremorgin $\mathrm{B}$ derivatives<smiles>COc1ccc2c(C[C@@H]3NC(=O)[C@@H]4CCCN4C3=O)c(C(=O)C=C(C)C)[nH]c2c1</smiles>

(226) 18-oxotryprostatin A<smiles>[R1]N1C(=O)[C@]2(c3ccc(OC)cc31)[C@H](C=C(C)C)N1C(=O)[C@@]3([R2])CCCN3C(=O)[C@@]1(O)[C@@H]2O</smiles>

(218) spirotryprostatin C $\mathrm{R}^{1}=$ 刍 $R^{2}=H$

(219) spirotryprostatin $D$ $\mathrm{R}^{1}=$<smiles>[Z20]CC=C(C)C</smiles>

(220) spirotryprostatin $E$ $\mathrm{R}^{1}=$<smiles>[R]=C=CC(C)(C)OO</smiles>

(224) spirotryprostatin $\mathrm{F}$ $\mathrm{R}^{1,2}=\mathrm{H}$<smiles>CC(C)=CCc1cccc2c(C[C@@H]3C(=O)N[C@H](C)C(=O)N3O)c[nH]c12</smiles>

(227) 14-hydroxyterezine D<smiles>COc1ccc2c3c4n(c2c1)[C@H](C)[C@@H](C=C(C)C)OOC(C)(C)C[C@H]4N1C(=O)[C@H]2CCCN2C(=O)[C@]1(O)C3=O</smiles>

(223) 13-oxoverruculogen<smiles>COc1ccc2c(c1)NC(=O)[C@]21C=C2C(=O)N3CCC[C@H]3C(=O)N2[C@H]1C=C(C)C</smiles>

(225) 6-methoxyspirotryprostatin B<smiles>COc1ccc2c3c([nH]c2c1)[C@@H](OC)[C@@]1(OC)C(=O)N2CCC[C@H]2C(=O)N1[C@H]3CC(C)(C)O</smiles>

(228) cyclotryprostatin E

Figure 50. Prenylated indole diketopiperazine alkaloids.<smiles>[R]C1c2c(c3ccc(OC)cc3n2[R])[C@@]2(O)C(=O)N3CCC[C@@H]3C(=O)N1[C@H]2C=C(C)C</smiles>

(229) $\mathrm{R}^{1}=$ prenyl , $\mathrm{R}^{2}=\mathrm{OMe}, \beta-\mathrm{OH}$ prenylcyclotryprostatin $\mathrm{B}$ (231) $\mathrm{R}^{1,2}=\mathrm{H}, \alpha-\mathrm{OH}$

9-hydroxyfumitremorgin C<smiles>COc1ccc2c3c([nH]c2c1)[C@@H](OC)[C@]1(OC)C(=O)N2CCC[C@H]2C(=O)N1[C@H]3CC(C)(C)O</smiles>

(230) 20-hydroxycyclotryprostatin B<smiles>CC(C)=CCc1[nH]c2cc(O)ccc2c1C[C@H]1NC(=O)[C@H]2CCCN2C1=O</smiles>

(232) 6-hydroxytryprostatin B

Figure 51. Prenylated indole diketopiperazine alkaloids.

24-Hydroxyverruculogen (233), 26-hydroxyverruculogen (234) and 13-O-prenyl-26hydroxyverruculogen (235) have been isolated from the marine sediment-derived fungus Penicillium brefeldianum SD-273 (Figure 52). None of them exhibited pronounced antibacterial or cytotoxic effects, but 13-O-prenyl-26-hydroxyverruculogen showed a potent insecticidal activity against brine shrimp (Artemia salina, $\mathrm{LC}_{50}$ — concentration which is lethal to $50 \%$ of the test organisms - $9.44 \mu \mathrm{M}$ ) [148]. 


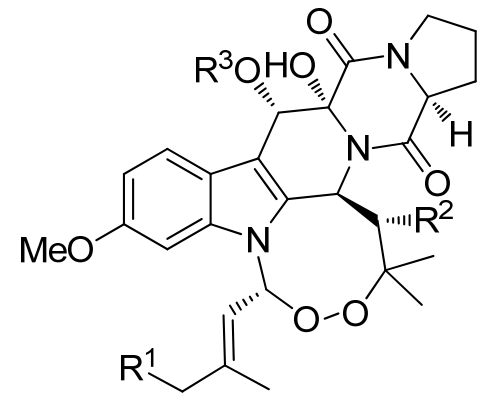

(233) $\mathrm{R}^{1}=\mathrm{OH}, \mathrm{R}^{2,3}=\mathrm{H}$

24-hydroxyverruculogen

(234) $\mathrm{R}^{1,3}=\mathrm{H}, \mathrm{R}^{2}=\mathrm{OH}$

26-hydroxyverruculogen

(235) $\mathrm{R}^{1}=\mathrm{H}, \mathrm{R}^{2}=\mathrm{OH}, \mathrm{R}^{3}=$ 给

13-O-prenyl-26-hydroxyverruculogen

Figure 52. Prenylated indole diketopiperazine alkaloids.

The diketopiperazine alkaloids carneamides $\mathrm{A}-\mathrm{C}(\mathbf{3 3 6}-\mathbf{3 3 8})$ were obtained from the marine-derived fungus Aspergillus carneus (Trichocomaceae) KMM 4638 (Figure 53). Carneamides B (337) and C (338) bear the rare indoloazocine subunit [149]. Dihydrocarneamide A (339), together with iso-notoamide B (200), was isolated from the marine-derived endophytic fungus Paecilomyces variotii EN-291. Both substances showed weak cytotoxic activity against NCI-H460 tumor cells ( IC $_{50} 69.3$ and $55.9 \mu \mathrm{mol} / \mathrm{L}$, respectively) [134].<smiles>C=CC(C)(C)c1[nH]c2cc3c(cc2c1C[C@H]1NC(=O)C2=CCCN2C1=O)C=CC(C)(C)O3</smiles>

(336) carneamide A<smiles>C=CC(C)(C)c1[nH]c2cc3c(cc2c1C[C@@H]1NC(=O)[C@H]2CCCN2C1=O)C=CC(C)(C)O3</smiles>

(339) dihydrocarneamide $\mathrm{A}$<smiles></smiles>

(337) carneamide B<smiles>CC1(C)C=Cc2cc3c4c([nH]c3cc2O1)C(C)(C)/C=C\N1C(=O)[C@H]2CCCN2C(=O)[C@H]1C4</smiles>

(338) carneamide C

Figure 53. Carneamides $\mathrm{A}-\mathrm{C}$ and dihydrocarneamide $\mathrm{A}$.

From A. versicolor HDN08-60, eight prenylated indole diketopiperazines, versicamides A-H (340-347), were obtained, all being structurally related to a singly prenylated Trp-Pro diketopiperazine (Figure 54). The cytotoxicities of versicamides A-H were tested against the HeLa, HCT-116, HL-60, and K562 cell lines, but only versicamide H (347) showed moderate cytotoxic activity (IC50 19.4, 17.7, 8.7 and $22.4 \mu \mathrm{M}$, respectively) and PTK (protein tyrosine kinase) inhibitory activities [150]. 
<smiles>CC1(C)C=Cc2c(ccc3c4c([nH]c23)C(C)(C)/C=C\N2C(=O)C3=CCCN3C(=O)[C@H]2C4)O1</smiles>

(340) versicamide A<smiles>CC1(C)C=Cc2c(ccc3c2N=C2C(C)(C)/C=C\N4C(=O)C5=CCCN5C(=O)[C@@H]4C[C@@]23O)O1</smiles>

(343) versicamide D<smiles>Cc1c(C(=O)NC2=C(NC(=O)C(C)(C)C)C=CN3C(=O)C4=CCCN4C(=O)[C@@H]23)ccc2c1C=CC(C)(C)O2</smiles>

(346) versicamide G<smiles>COC1CC(C)(C)Oc2ccc3c4c([nH]c3c21)C(C)(C)C=CN1C(=O)C2=CCCN2C(=O)[C@@H]1C4</smiles>

(341) versicamide B<smiles></smiles>

(344) versicamide $E$<smiles>COc1c2c(nc3c4c(ccc13)OC(C)(C)C=C4)C(C)(C)C=CN1C(=O)C3=CCCN3C(=O)[C@@H]21</smiles>

(347) versicamide $\mathrm{H}$<smiles></smiles>

(342) versicamide C<smiles></smiles>

(345) versicamide F

Figure 54. Versicamides A-H.

Brevianamides S-V (348-351) and 9Z-O-2(2,3-dimethylbut-3-enyl)brevianamide Q (352) were obtained from strains of Aspergillus versicolor, isolated from sediment collected from the Bohai Sea and brown alga Sargassum thunbergii, respectively (Figure 55). Brevianamide S (348) displayed selective antibacterial activity against Bacille Calmette-Guérin (BCG) which was used as a live attenuated vaccine against tuberculosis [151,152]. Furthermore, brevianamide $\mathrm{W}(\mathbf{3 5 3})$ was isolated from A. versicolor CXCTD-06-6a. It showed moderate radical scavenging activity towards DPPH, but no cytotoxic effects [153].

The halotolerant fungus Aspergillus variecolor was the source of variecolorins A-L (354-365) (Figure 56) [154]. In contrast, the structurally related variecolorins $\mathrm{M}-\mathrm{O}(\mathbf{3 6 6}-\mathbf{3 6 8})$ were isolated from a deep-ocean sediment-derived fungus, Penicillium griseofulvum [155]. Variecolorin G (360) exhibited moderate lethal activity against brine shrimp ( $\mathrm{LC}_{50} 42.6 \mu \mathrm{g} / \mathrm{mL}$ ) [156]. Variecolorins A-K (354-364) and $\mathrm{M}-\mathrm{O}(\mathbf{3 6 6}-\mathbf{3 6 8})$ displayed weak DPPH radical scavenging activity, variecolorins A-O (354-368) did not show cytotoxic effects against the P388, HL-60, BEL-7402 and A549 cell lines $\left(\mathrm{IC}_{50}>50 \mu \mathrm{M}\right)[154,155]$.

Variecolortides A-C (369-371) were isolated from Aspergillus variecolor B-17, obtained from sediments of the Mongolian Jilantai Salt Field, China (Figure 57). They displayed weak cytotoxicity towards the K562 human leukemia cell line (IC50 61, 69 and $71 \mu \mathrm{M}$ ) and exhibited weak DPPH-radical scavenging activities [157]. 7-O-Methylvariecolortide A (372) was isolated from the mangrove-derived Eurotium rubrum [158]. Variecolortides also showed caspase-3 inhibitory activity [159]. 


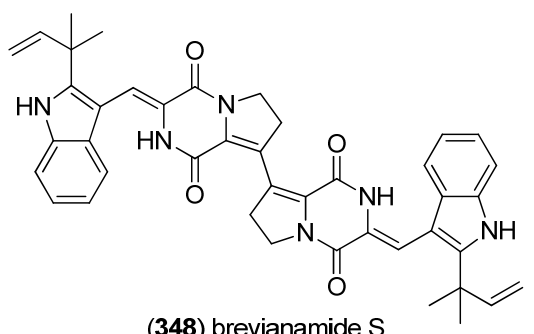

(348) brevianamide $S$

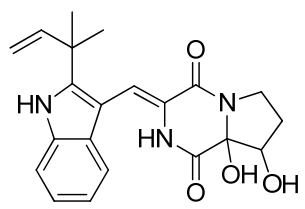

(350) brevianamide U

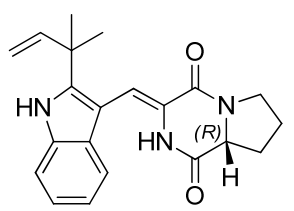

(353) brevianamide W

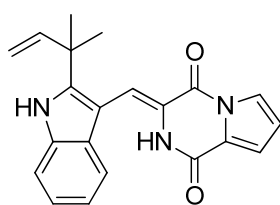

(349) brevianamide T

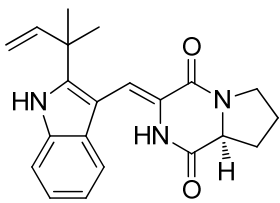

(351) brevianamide $\mathrm{V}$

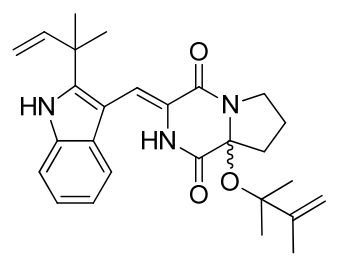

(352) 9Z-O-2(2,3-dimethylbut -3-enyl)brevianamide $Q$

Figure 55. Brevianamides.

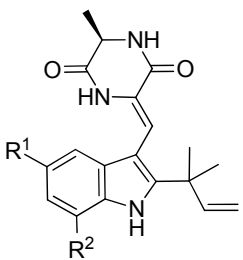

(354) variecolorin A

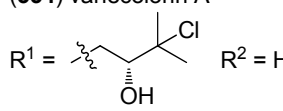
(355) variecolorin $\mathrm{B}$

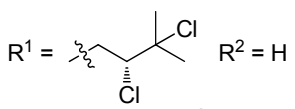
(356) variecolorin $\mathrm{C}$

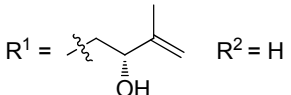
(357) variecolorin D $\mathrm{R}^{1}=$ (358) variecolorin $\mathrm{E}$

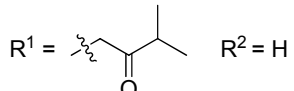
(359) variecolorin $\mathrm{F}$<smiles>[R]=C=CC[C@H](O)C(C)(C)Cl</smiles>
(360) variecolorin $\mathrm{G}$ $\mathrm{R}^{1}=\mathrm{H}, \mathrm{R}^{2}=$ 玲

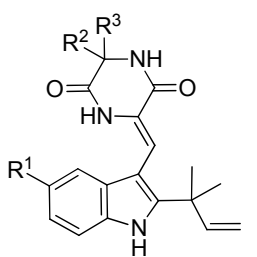

(361) variecolorin $\mathrm{H}$

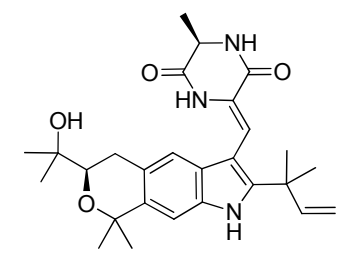

(364) variecolorin $\mathrm{K}$

$\mathrm{R}^{1}=\mathrm{H}, \mathrm{R}^{2}=\mathrm{Me}, \mathrm{R}^{3}=\mathrm{OMe}$

(362) variecolorin

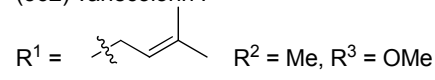

(363) variecolorin J

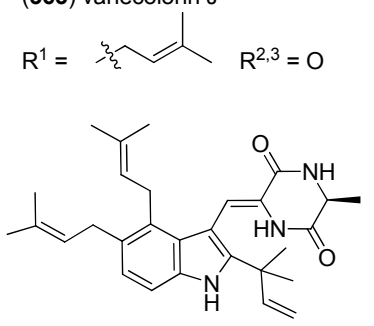

(365) variecolorin L

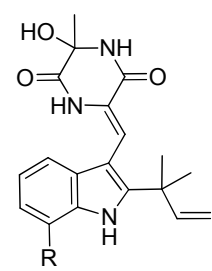

(368) variecolorin O

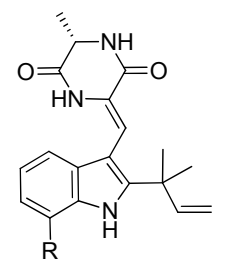

(366) variecolorin M $\mathrm{R}=$ 年 $\mathrm{OH}$

(367) variecolorin $\mathrm{N}$

$\mathrm{R}=$ s $\mathrm{OH}$

Figure 56. Variecolorins $\mathrm{M}-\mathrm{O}$. 


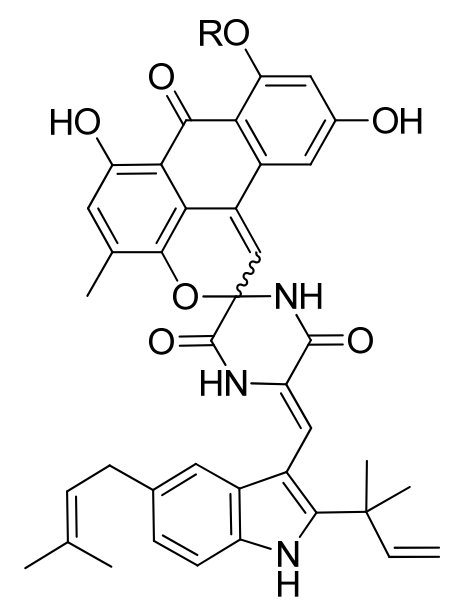

(369) $\mathrm{R}=\mathrm{H}$ variecolortide $\mathrm{A}$

(372) $\mathrm{R}=$ Me 7-O-methylvariecolortide A

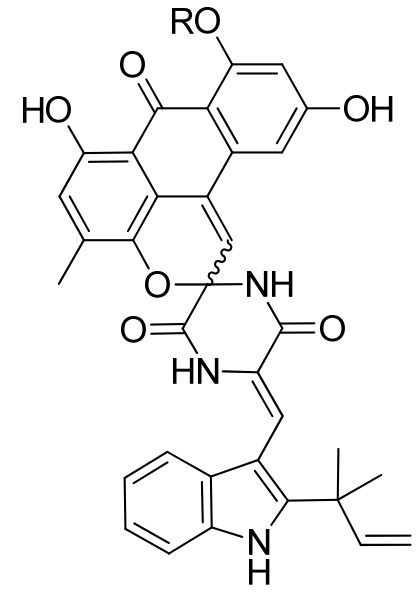

(370) $\mathrm{R}=\mathrm{H}$ variecolortide $\mathrm{B}$

(371) $\mathrm{R}=$ Me variecolortide $\mathrm{C}$

Figure 57. Variecolortides.

Examination of the fungal strain Eurotium rubrum, an endophytic fungus isolated from the mangrove plant Hibiscus tiliaceus, resulted in the isolation of two dioxopiperazine derivatives, dehydrovariecolorin L (373) and dehydroechinulin (374) (Figure 58). They neither showed radical scavenging nor cytotoxic activity towards the P388, HL-60 and A549 cell lines [160]. 12-Demethyl12-oxo-eurotechinulin B (375) was isolated from the same organism and showed cytotoxic activity against the SMMC-7721 tumor cell line ( $\mathrm{IC}_{50} 30 \mu \mathrm{g} / \mathrm{mL}$ ) [161].<smiles>C=CC(C)(C)c1[nH]c2ccc(CC=C(C)C)c(CC=C(C)C)c2c1/C=C1\NC(=O)[C@H](C)NC1=O</smiles>

(373) dehydrovariecolorin L

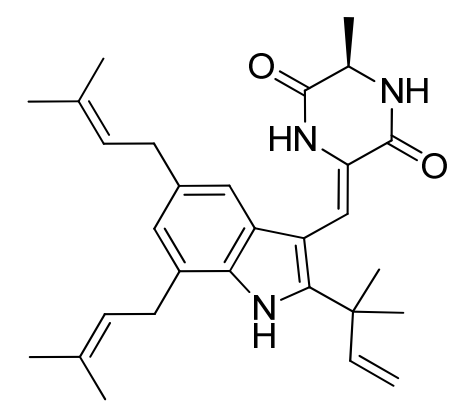

(374) dehydroechinulin<smiles>C=CC(C)(C)c1[nH]c2c(CC=C(C)C)cc(CC=C(C)C)cc2c1C=C1NC(=O)C(=O)NC1=O</smiles>

(375) 12-demethyl-12-oxoeurotechinulin B

Figure 58. Dehydrovariecolorin L, dehydroechinulin and 12-demethyl-12-oxo-eurotechinulin B.

Further investigation of Eurotium rubrum revealed fifteen new prenylated indole diketopiperazine alkaloids, named rubrumlines A-O (376-390) (Figure 59). They were tested against the influenza A/WSN/33 virus and showed variable antiviral activities, governed by their substitution pattern and the presence of the $\Delta^{8,9}$ double bond [162]. 


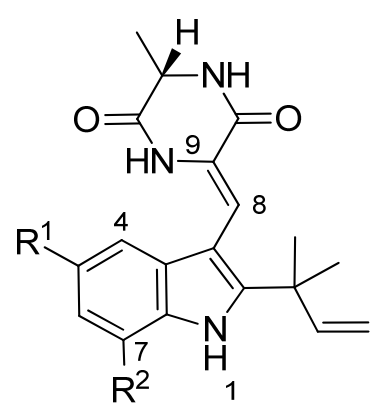

(376) rubrumline A

$\mathrm{R}^{1}=$

$\mathrm{R}^{2}=\mathrm{H}$

(377) rubrumline $B$

$\mathrm{R}^{1}=$

$\mathrm{R}^{2}=\mathrm{H} \quad \mathrm{OH}$

(378) rubrumline $C$

$\mathrm{R}^{1}=\mathrm{H}$

(383) rubrumline $\mathrm{H}$

$\mathrm{R}^{1}=\mathrm{H}$

$\mathrm{R}^{2}=\xi_{\mathrm{OH}}^{z_{2}} \mathrm{OH}$

(384) rubrumline I

$\mathrm{R}^{1}=\mathrm{H}$

$\mathrm{R}^{2}=$ 旅<smiles>[R]c1cc([R2])c2[nH]c(C(C)(C)C=C)c(/C=c3\[nH]c(=O)c(=C)[nH]c3=O)c2c1</smiles>

(379) rubrumline D

$\mathrm{R}^{1}=$<smiles>[R][CH][Z20]([H])=C[C@H](O)CO</smiles>

(380) rubrumline $E$

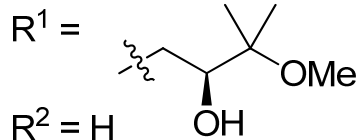<smiles>[R7]c1cc([R2])c2[nH]c(C(C)(C)C=C)c(C[C@H]3NC(=O)[C@H](C)NC3=O)c2c1</smiles>

(381) rubrumline $F$ $\mathrm{R}^{1}=$<smiles>[R]=C[14CH2][14CH2][C@H](O)C(OC)OC</smiles>

(382) rubrumline $\mathrm{G}$

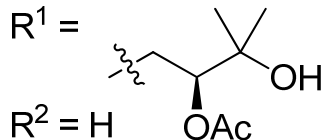<smiles>C=CC(C)(C)c1[nH]c2ccccc2c1C[C@H]1NC(=O)[C@H](CO)NC1=O</smiles>

(385) rubrumline $\mathrm{J}$<smiles>C=CC(C)(C)c1[nH]c2ccccc2c1/C=C1\NC(=O)[C@](O)(CO)NC1=O</smiles>

(386) rubrumline $K$<smiles>[R]c1ccc2[nH]c(C(C)(C)C=C)c(/C=C3\NC(=O)C(=O)NC3=O)c2c1</smiles>

(390) rubrumline $O$<smiles>[R]c1cc([R2])c2[nH]c(C(C)(C)C=C)c(C(O)[C@H]3NC(=O)[C@H](C)NC3=O)c2c1</smiles>

(388) rubrumline $M$ $\mathrm{R}=\mathrm{H}$

(389) rubrumline $\mathrm{N}$ $\mathrm{R}=\mathrm{Me}$

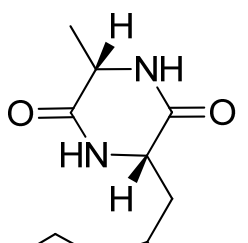<smiles>C=CC(C)(C)n1cc(C)c2ccccc21</smiles>

Figure 59. Rubrumlines A-O.

Further isoechinulin type alkaloids, rubrumazines A-C (391-393), have been obtained from Eurotium rubrum MA-150 (Figure 60). Rubrumazine B (392) displayed potent activity (LC $\mathrm{L}_{50} 2.43 \mu \mathrm{M}$ ) against brine shrimp (Artemia salina), while rubrumazine A (391) and C (393) showed only modest activity ( $\mathrm{LC}_{50} 29.8$ and $16.5 \mu \mathrm{M}$, respectively) in the same assay. Rubrumazines did not show activity in antibacterial screening [163]. 
<smiles></smiles>

(391) rubrumazine $\mathrm{A}$<smiles>[R]=C=CCC(O)C(C)(C)OC</smiles>

(392) rubrumazine $\mathrm{B}$<smiles>[R]=C[Y]C(O)C(C)(C)O</smiles>

(393) rubrumazine $\mathrm{C}$<smiles>[R][C@H](O)[C@H](O)[C@](C)(O)[R]#[R]</smiles>

Figure 60. Rubrumazines A-C.

Effusin A (394) and dihydrocryptoechinulin D (395) were isolated from a mangrove-derived fungus, Aspergillus effuses H1-1 (Figure 61). Dihydrocryptoechinulin D (395) showed cytotoxicity towards P388 and HL-60 cells lines and inhibitory activity on topoisomerase I [164]. Dihydroneochinulin B (396) was also isolated from A. effuses H1-1 and showed weak cytotoxicity against BEL-7402 and A549 tumor cell lines (IC50 55.1 and $30.5 \mu \mathrm{M}$ ) [165].

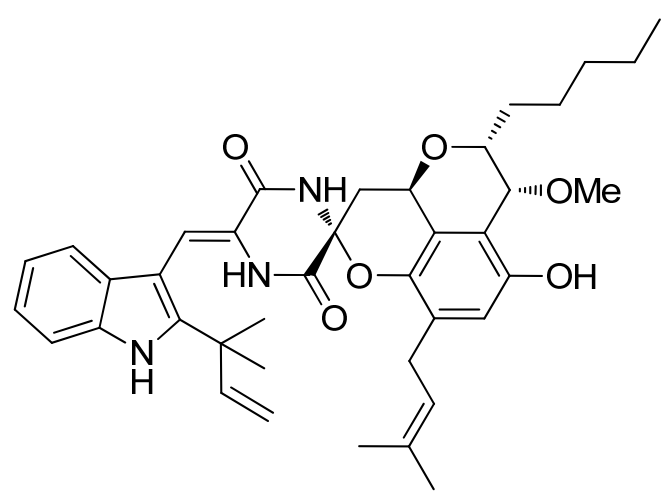

(394) effusin A<smiles>C=CC(C)(C)c1[nH]c2ccccc2c1C[C@H]1NC(=O)C(=C)NC1=O</smiles>

(396) dihydroneochinulin B<smiles>C=CC(C)(C)c1[nH]c2ccccc2c1/C=C1\NC(=O)C2(CC(C)C=CC2CCc2c(O)cc(CC=C(C)C)c(O)c2C=O)NC1=O</smiles>

(395) dihydrocryptoechinulin D

Figure 61. Effusin A, dihydrocryptoechinulin D and dihydroneochinulin B.

Cristatumins A-D (397-400) [156] and F (401) [166] were identified from the culture extract of Eurotium cristatum EN-220, an endophytic fungus isolated from the marine alga Sargassum thunbergii (Figure 62). Cristatumin A (397) exhibited antibacterial activity against Escherichia coli (MIC — minimum 
inhibitory concentration - $64.8 \mu \mathrm{g} / \mathrm{mL})$, while cristatumin B (398) showed moderate activity against brine shrimp (LC50 $74.4 \mu \mathrm{g} / \mathrm{mL}$ ) [156]. Cristatumin F (401) exhibits a valine unit in its diketopiperazine structure, which is unprecedented. It also shows modest radical scavenging activity towards DPPH and a marginal cell proliferation inhibition [166]. Eurocristatine (402) was also isolated from E. cristatum and did not show any appreciable cytotoxic, antibacterial or antifungal activity [167].

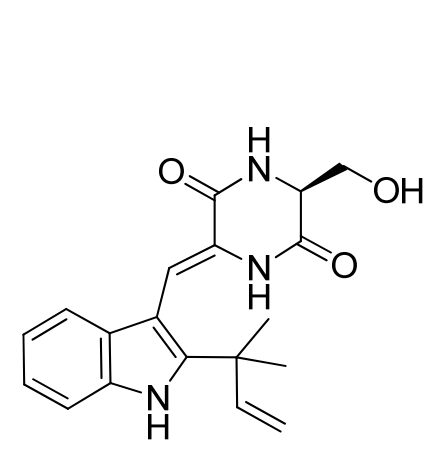

(397) cristatumin A

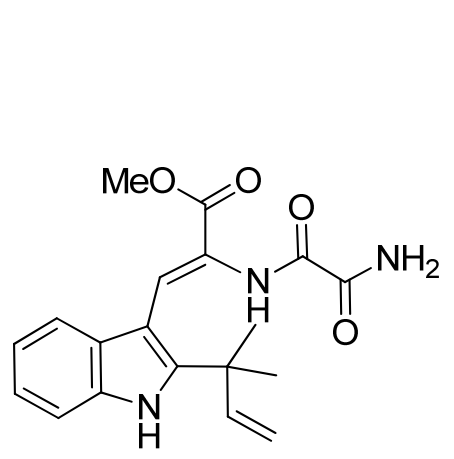

(400) cristatumin D

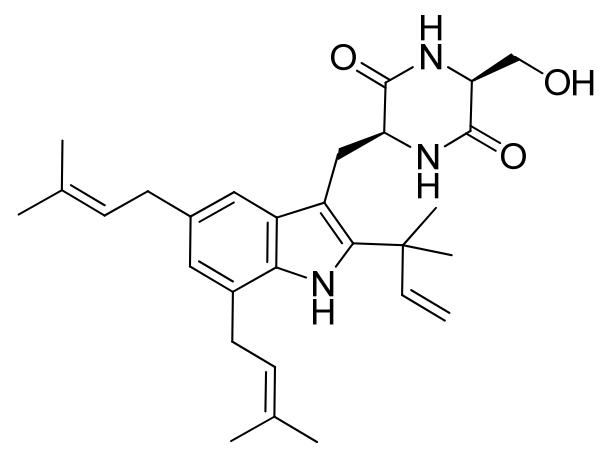

(398) cristatumin B

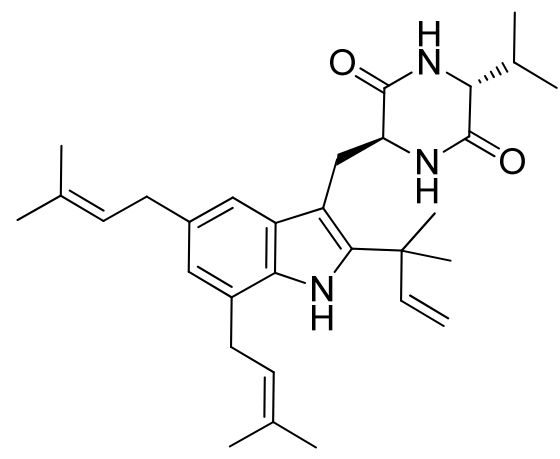

(401) cristatumin F

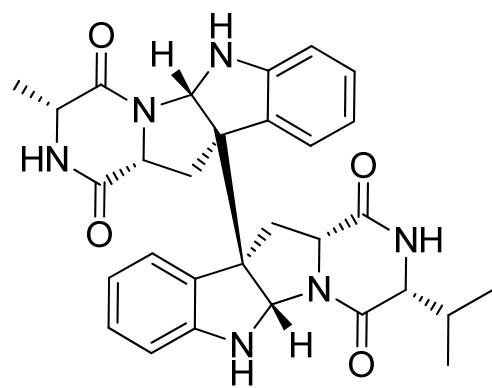

(399) cristatumin C

Figure 62. Cristatumins A-F and eurocristatine.

The diketopiperazine alkaloids brevicompanines A (403), B (404)[168,169], C (405) [170] and D-H (406-410) [171] have been isolated from the fungus Penicillium brevicompactum, as well as Aspergillus janus, Penicillium brevi-compactum Dierckx and Penicillium sp. F1, respectively (Figure 63). Brevicompanine B (404) showed antiplasmodial activity against the malaria parasite Plasmodium falciparum 3D7 (IC50 $35 \mathrm{mg} / \mathrm{mL}$, compound precipitated in the test media), but no antifungal or antibacterial effects [168]. Brevicompanines A-C (403-404) were found to be plant growth regulators [169,170], whereas brevicompanines D-H (406-410) showed inhibitory effects on lipopolysaccharide-induced inflammation in BV2 microglial cell lines [171]. 
<smiles>C=CC(C)(C)[C@]12C[C@@H]3C(=O)N[C@H](C(C)CC)C(=O)N3[C@H]1Nc1ccccc12</smiles>

(403) brevicompanine A

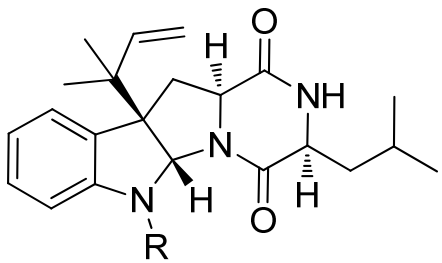<smiles>C=CC(C)(C)[C@]12C[C@H]3C(=O)N[C@H](CC(C)C)C(=O)N3[C@H]1Nc1ccccc12</smiles>

(404) brevicompanine B

(406) brevicompanine D

$$
\mathrm{R}=\text { - }
$$

(407) brevicompanine E<smiles>[R][Y6](=O)CCC</smiles>

(408) brevicompanine F

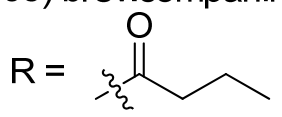<smiles>C=CC(C)(C)[C@]12C[C@H]3C(=O)N[C@H](C(C)C)C(=O)N3[C@H]1Nc1ccccc12</smiles>

(405) brevicompanine C<smiles>[R]N1c2ccccc2[C@@]2(C(C)(C)C=C)C[C@@H]3C(=O)N[C@@]([CH]C(C)C)(C(C)C)C(=O)N3[C@H]12</smiles>

(409) brevicompanine G<smiles>[R][Y5](=O)C(C)=O</smiles>

(410) brevicompanine $\mathrm{H}$<smiles></smiles>

Figure 63. Brevicompanines A-H.

Nocardioazines A (411) and B (412) have been isolated from an Australian marine sediment-derived bacterium, Nocardiopsis sp. (CMB-M0232) (Figure 64). Nocardioazine A (411) was found to be an effective and noncytotoxic inhibitor of the multidrug resistance factor P-glycoprotein and is able to reverse doxorubicin resistance in MDR (multi-drug resistant) SW620 Ad300 cells [172].

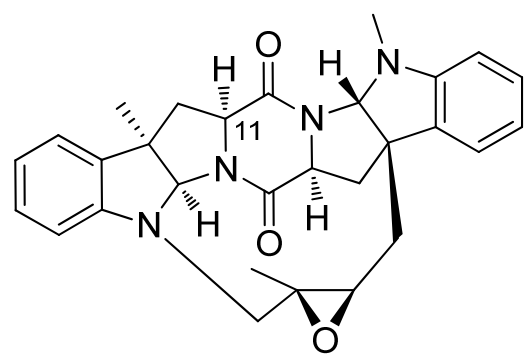

(411) nocardioazine A<smiles>CC(C)=CC[C@@]12C[C@@H](C(=O)N3CC[C@]4(C)c5ccccc5N[C@@H]34)[C@@H](N(C)c3ccccc31)N2C</smiles>

(412) nocardioazine B

Figure 64. Nocardioazines A and B.

The drimane-substituted tryptophan-derived diketopiperazines indotertines A (413) and B (414) and drimentines $\mathrm{F}-\mathrm{H}$ (415-417) were isolated from the marine sediment-derived actinomycete Streptomyces sp. CHQ-64 (Figure 65). Drimentine G (416) exhibited cytotoxic activities against HCT-8, Bel-7402, A549, and A2780 cell lines ( $\mathrm{IC}_{50} 2.81,1.38,1.01$, and $2.54 \mu \mathrm{M}$, respectively) together with a weak topoisomerase I inhibitory activity. Indotertine B (414) showed cytotoxic activities against the two human tumor cell lines HCT-8 and A549 (IC50 6.96 and $4.88 \mu \mathrm{M}$, respectively) [173,174]. 


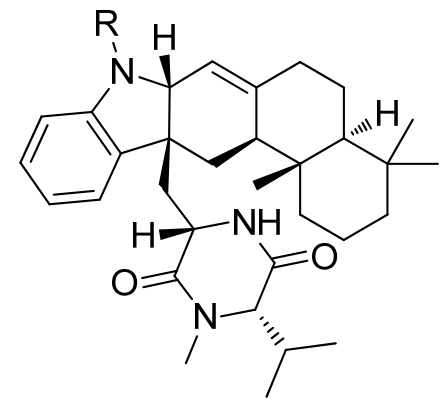

(413) $\mathrm{R}=\mathrm{H} \quad$ indotertine $\mathrm{A}$

(414) $\mathrm{R}=\mathrm{CHO}$ indotertine $\mathrm{B}$

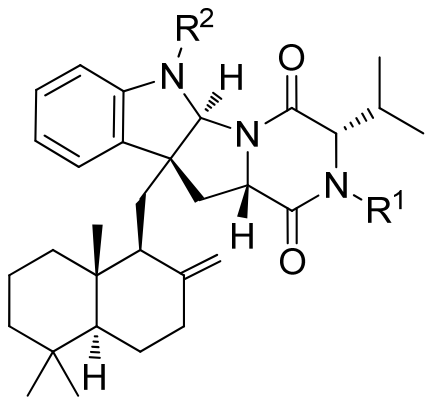

(415) $\mathrm{R}^{1}=\mathrm{Me}, \mathrm{R}^{2}=\mathrm{H} \quad$ drimentine $\mathrm{F}$

(416) $\mathrm{R}^{1,2}=\mathrm{H} \quad$ drimentine $\mathrm{G}$

(417) $\mathrm{R}^{1}=\mathrm{Me}, \mathrm{R}^{2}=\mathrm{CHO}$ drimentine $\mathrm{H}$

Figure 65. Indotertines $\mathrm{A}$ and $\mathrm{B}$ and drimentines $\mathrm{F}-\mathrm{H}$.

Okaramines S-U (418-420) were produced by Aspergillus taichungensis ZHN-7-07, isolated from the rhizosphere soil of the mangrove plant Acrostichum aureum (Figure 66). Okaramine S (418) showed cytotoxic activity against HL-60 and K562 cell lines ( $\mathrm{IC}_{50} 0.78$ and $22.4 \mu \mathrm{M}$, respectively), but none of them exhibited antibiotic activities [175].<smiles>[R]c1cccc2c1N[C@H]1N3C(=O)[C@H](Cc4c[nH]c5c([R])cccc45)NC(=O)[C@@H]3C[C@]21O</smiles><smiles>[R12]#CCCCC=C(C)C</smiles>
(419) $R^{1}=$ - 2 R $R^{2}=H$ okaramine $T$ (420) $\mathrm{R}^{1,2}=\mathrm{H} \quad$ okaramine $\mathrm{U}$

Figure 66. Okaramines S-U.

The diketomorpholine shornephine A (421) was isolated from an Australian marine sediment-derived Aspergillus sp. (CMB-M081F) and identified as a noncytotoxic inhibitor of P-glycoprotein-mediated drug efflux in human MDR cancer cells (Figure 67) [176].<smiles>C=CC(C)(C)[C@]12Nc3c(O)cccc3[C@@]1(O)C[C@H]1C(=O)O[C@@H](Cc3ccccc3)C(=O)N12</smiles>

(421) shornephine A

Figure 67. Shornephine A. 
The communesins are a class of cytotoxic and insecticidal marine indole alkaloids [177-179]. The first representatives, communesin A (422) and B (423), have been isolated in 1993 from the mycelium of a strain of Penicillium sp. found on the marine alga Enteromorpha intestinalis and displayed cytotoxic activity against leukemia cell line P388 (Figure 68) [177]. The extract of Penicillium sp., derived from the Mediterranean sponge Axinella verrucosa, yielded new derivatives communesins $\mathrm{C}$ (424) and D (425), which exhibit moderate antiproliferative activity against leukemia cell lines U-937, THP-1, NAMALWA, L-428, MOLT-3, and SUP-B15 [179]. Communesins D (425), E (426) and F (427) (published as communesins $\mathrm{C}, \mathrm{D}$ and $\mathrm{E}$, communesin $\mathrm{C}$ turned out to be identical to communesin $\mathrm{D}$ published by Jadulco et al. [179], communesins $\mathrm{E}$ and $\mathrm{F}$ being new congeners) were isolated from the Japanese Penicillium expansum Link MK-57, together with communesins A and B. All of them showed insecticidal activity against silkworm larvae [178]. Communesins G (428) and H (429) have been isolated from the psychrotolerant fungus Penicillium rivulum Frisvad [180]. Studies of the biosynthetic pathway of the communesin alkaloids led to the identification of communesins I-K (430-432) and confirmed aurantioclavine as a biosynthetic precursor [181].

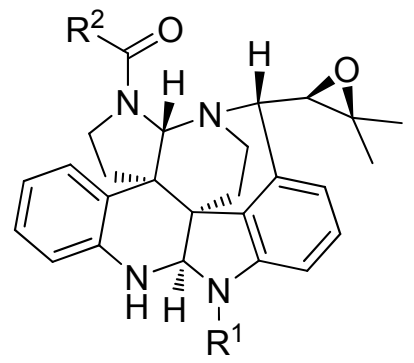
(422) $\mathrm{R}^{1,2}=$ Me communesin $\mathrm{A}$
(423) $R^{1}=\mathrm{Me} \quad \mathrm{R}^{2}=\quad$ communesin $\mathrm{B}$

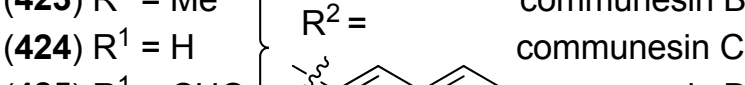
(425) $\mathrm{R}^{1}=\mathrm{CHO}$ communesin $\mathrm{D}$
(426) $R^{1}=H, R^{2}=$ Me communesin $E$
(428) $R^{1}=$ Me, $R^{2}=$ Et communesin $G$
(429) $\mathrm{R}^{1}=\mathrm{Me}, \mathrm{R}^{2}={ }^{n} \mathrm{Pr}$ communesin $\mathrm{H}$

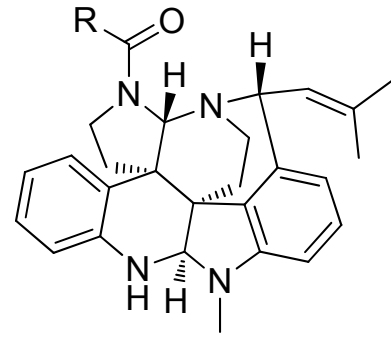

(427) $R=$ Me communesin $F$

(431) $R=$

communesin $\mathrm{J}$

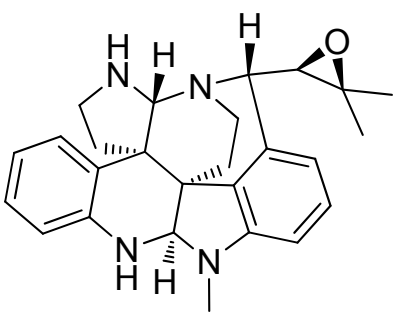

(430) communesin I

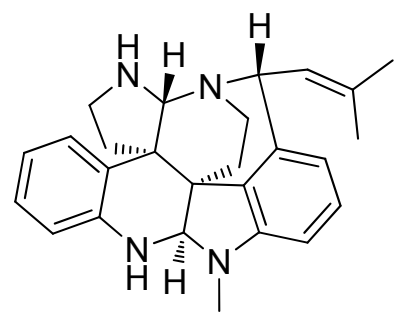

(432) communesin $\mathrm{K}$

Figure 68. Communesins $\mathrm{A}-\mathrm{K}$.

Since the shearinines A-C, the first representatives of their class, have been isolated from the sclerotioid ascostromata of Eupenicillium shearii [182]. examination of the endophytic fungus Penicillium sp., isolated from the mangrove plant Aegiceras corniculatum, led to the discovery of marine-derived shearinines D-K (433-440) (Figure 69) [183]. Simultaneously, three shearinines, named D, E (441), and F were isolated by another research group from the marine-derived strain of the fungus Penicillium janthinellum Biourge [184]. Shearinine D as published by Smetanina et al. was identical to shearinine $\mathrm{D}$ reported by Xu et al. [183], whereas shearinine F published by Smetanina et al. turned out to be identical to shearinine K (440) published by Xu et al. $[183,185]$. Shearinines A, D, and 
$\mathrm{E}$ were found to induce apoptosis in the human leukemia cell line HL-60, shearinine E also inhibits EGF-induced malignant transformation of JB6 $\mathrm{P}^{+} \mathrm{Cl} 41$ cells [184]. Shearinines A-C show insecticidal activity against Helicoverpa zea, Carpophilus hemipterus and Spodoptera frugiperda [182,186], whereas shearinines D, E and G exhibit inhibitory activity on large-conductance calcium-activated potassium channels [183]. Moreover, shearinines D and E were found to inhibit Candida albicans biofilm formation [187]. The biosynthesis of the shearinines H-J involves the cleavage of the 2,3-double bond of the indole ring, a phenomenon well known from tryptophan catabolism.

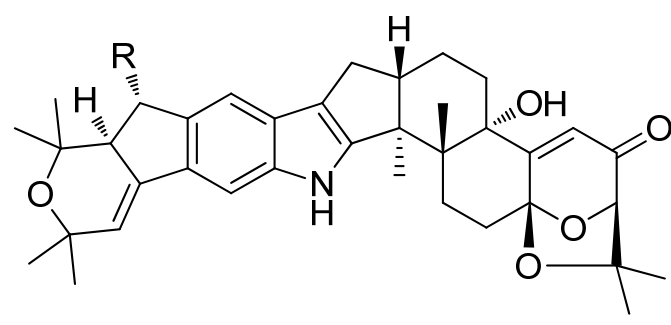

(433) $\mathrm{R}=\mathrm{OH}$ shearinine $\mathrm{D}$

(434) $\mathrm{R}=$ OMe shearinine $\mathrm{E}$

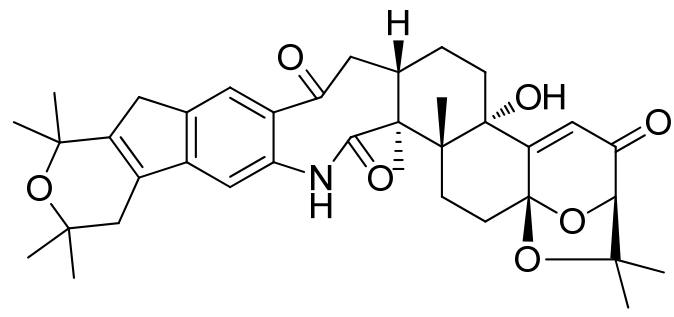

(437) shearinine $H$<smiles></smiles>

(439) shearinine $\mathrm{J}$

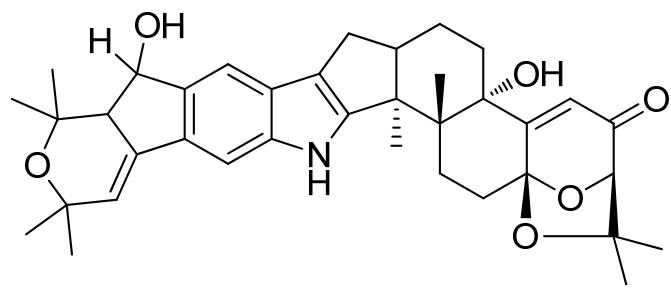

(441) $\left(\mathrm{H}_{22}, \mathrm{H}_{23}\right.$-cis) shearinine $\mathrm{E}$ (as reported by Smetanina et al.)

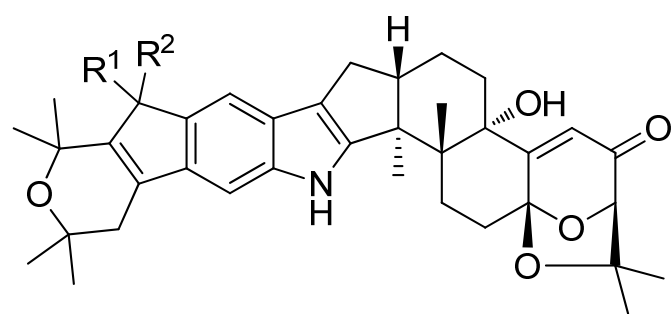

(435) $R^{1,2}=H$ shearinine $F$ (436) $R^{1,2}=O$ shearinine $G$<smiles>CC1(C)C=C2c3cc4c(cc3C[C@H]2C(C)(C)O1)C(=O)C[C@H]1CC[C@]2(O)C3=CC(=O)[C@H]5NC(=O)[C@]3(CC[C@]2(C)[C@@H]41)O5</smiles>

(438) shearinine I

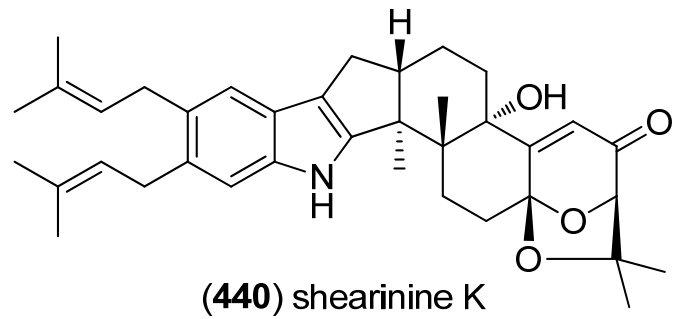

Figure 69. Shearinines D-K (nomenclature according to Xu et al.).

Asporyzins A-C (442-444) were obtained from an endophytic fungus Aspergillus oryzae, isolated from the marine red alga Heterosiphonia japonica and showed low acetylcholineesterase (AChE) modulating activity (Figure 70) [188]. 


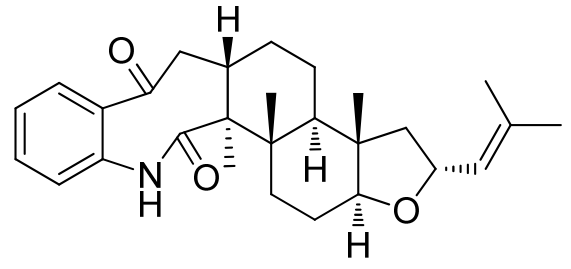

(442) asporyzin A<smiles>CC(C)(O)/C=C\C[C@]1(C)C(O)CC[C@@]2(C)[C@H]1CC[C@H]1CC3c4ccccc4NC3[C@]12C</smiles>

(444) asporyzin C

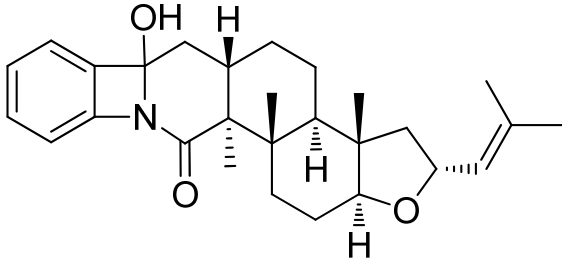

(443) asporyzin B

Figure 70. Asporyzins A-C.

The spirocyclic citrinadins A (445) [189] and B (446) were isolated from Penicillium citrinum, obtained from a marine red alga and their absolute configuration was elucidated via ROESY correlations, electronic circular dichroism (ECD), and vibrational circular dichroism (VCD) (Figure 71). Citrinadin B (446) showed cytotoxic acitivity against murine leukemia L1210 cells (IC50 $10 \mu \mathrm{g} / \mathrm{mL})$ [190].<smiles></smiles>

(445) citrinadin A<smiles>CN[C@]12CN3[C@@H](C)CCC[C@H]3C[C@]1(O)[C@](C)(O)[C@@]1(C2)C(=O)Nc2c(C(=O)C3O[C@@H]3C)cccc21</smiles>

(446) citrinadin B

Figure 71. Citrinadins $A$ and $B$.

The teleocidin analog 14- $O$-( $N$-acetylglucosaminyl) teleocidin A (GlcNAc-TA) (447) was isolated from Streptomyces sp. MM216-87F4 and was shown to affect the release of the neurotransmitter substance P from Dorsal Root Ganglia (DRG) neurons via protein kinase C (PKC) pathway (Figure 72) [191]. JBIR-31 (448) was isolated from Streptomyces sp. NBRC 105896, obtained from the marine sponge Haliclona sp. (Tateyama, Japan) and exhibited cytotoxicity towards HeLa and ACC-MESO-1 cell lines (IC50 49 and $88 \mu \mathrm{M}$, respectively) [192]. Extraction of Moorea producens, collected from Hawaii, afforded lyngbyatoxin derivatives, 12-epi-lyngbyatoxin A (449), 2-oxo-3(R)hydroxy-lyngbyatoxin A (450) and 2-oxo-3(R)-hydroxy-13- $N$-demethyl-lyngbyatoxin A (451) [193,194].

Indolactam alkaloids, 13- $N$-demethyl-methylpendolmycin (452) and methylpendolmycin-14-O- $\alpha$ glucoside (453) were isolated from the actinomycete Marinactinospora thermotolerans SCSIO 00652. They did not exhibit cytotoxic activities ( $\mathrm{IC}_{50}>50 \mu \mathrm{M}$ ), but showed antiplasmodial activities against Plasmodium falciparum strains 3D7 and Dd2 (IC50 1.92-36.03 $\mu \mathrm{M})$ [195]. 
<smiles>C=CC(C)(CCC=C(C)C)c1ccc(N(C)C(C(=O)NC(CCCCCC)COC2OC(CO)C(O)C(O)C2N)C(C)C)c2[nH]cc(CCCCC)c12</smiles>

(447) GIcNAc-TA<smiles></smiles>

(449) 12-epi-lyngbyatoxin A<smiles>[R]C[C@@H](Cc1c[nH]c2c(C(C)(C)C=C)ccc(N([R])[C@@H](C)[C@@H](C)CC)c12)NC(=O)C(C)C</smiles><smiles></smiles>

(448) 2-oxo-telocidin $A_{1}$ (JBIR-31)<smiles>[R]N(c1ccc([C@](C)(C=C)CCC=C(C)C)c2c1NC(=O)[C@H]2O)[C@@H](C(=O)N[C@@H](CC)CO)C(C)C</smiles>

(450) $\mathrm{R}=\mathrm{Me}$

(451) $\mathrm{R}=\mathrm{H}$

(452) $R^{1,2}=H$ 13-N-demethyl-methylpendolmycin

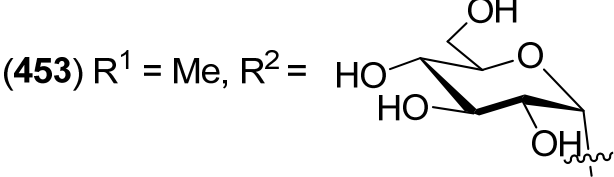

methylpendolmycin-14-O- $\alpha$-glucoside

Figure 72. Teleocidin and pendolmycin analogs.

Meleagrins B-E (454-457) and diketopiperazines, roquefortines F-I (458-461), have been isolated from the deep-sea derived fungus Penicillium sp. (Figure 73). Meleagrin B (454) showed cytotoxicity against HL-60, MOLT-4, A549 and BEL-7402 cell lines. Likewise, the structurally less complex Maleagrins D (456) and E (457) were moderately cytotoxic against A549 cell line (IC50 32.2 and $55.9 \mu \mathrm{M})[196,197]$.

Xiamycin A (462) and xiamycin A methyl ester (463), pentacyclic carbazole derivatives, were isolated from the endophytic Streptomyces sp. GT2002/1503 (from B. gymnorrhiza) (Figure 74). Xiamycin A (462) was found to be a selective anti-HIV agent by blocking R5-tropic viruses, without having effects on X4-tropic HIV-1 infections [198]. Xiamycin B (464) and indosespene (465) were isolated from the bacterial mangrove-derived endophyte Streptomyces sp. HKI0595 and exhibited strong antibacterial, but no cytotoxic activities against human tumor cell lines [199]. The atropisomeric N-N-linked dimers dixiamycins A (466) and B (467), oxiamycin (468), and chloroxiamycin (469) were isolated from a marine-derived actinomycete Streptomyces sp. SCSIO 02999. They exhibit 
antibacterial activity, the two dimeric metabolites being most active [200]. Prexiamycin (470) and preindosespene (471) were identified as intermediates in the xiamycin biosynthesis [15].

With 6-bromopenitrem B (472), a new penitrem derivative was isolated from the marine-derived fungus Penicillium commune isolate GS20 (supplemented with potassium bromide) (Figure 75). It showed significant anti-invasive effects, as well as antiproliferative activity against MCF-7 and MDA-MB-231 tumor cell lines [201]. 19-Hydroxypenitrem A (473) and 19-hydroxypenitrem E (474) were isolated from the endophytic marine red alga derived fungus Aspergillus nidulans EN-330 . Both compounds showed brine shrimp cytotoxicity (LD50 3.2 and $4.6 \mu \mathrm{M}$ ) and 19-hydroxypenitrem A (473) exhibited antimicrobial activity towards Edwardsiella tarda, Vibrio anguillarum, Escherichia coli and Staphylococcus aureus (MIC 16, 32, 16, and $16 \mu \mathrm{g} / \mathrm{mL}$, respectively) [202].

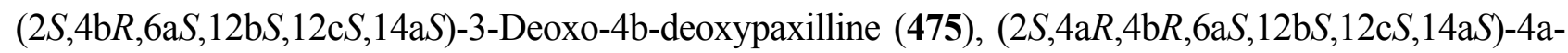

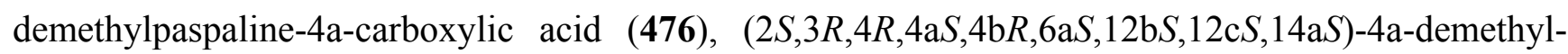

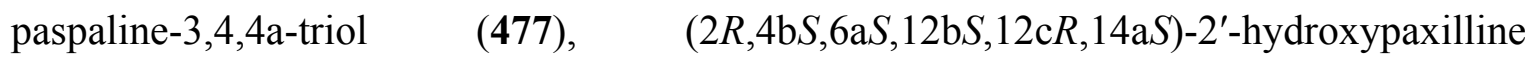
$(2 R, 4 \mathrm{~b} S, 6 \mathrm{a} S, 12 \mathrm{~b} S, 12 \mathrm{c} R, 14 \mathrm{a} S)-9,10$-diisopentenylpaxilline (479) and $(6 S, 7 R, 10 E, 14 E)-16$-(1H-indol-3yl)-2,6,10,14-tetramethylhexadeca-2,10,14-triene-6,7-diol (480) were isolated from the marine-derived Penicillium camemberti OUCMDZ-1492. Some compounds exhibited activity against $\mathrm{H}_{1} \mathrm{~N}_{1}$ influenza A virus (Figure 76) [203].

Emindole SB beta-mannoside (481) and 27-O-methylasporyzin C (482) were isolated from a marine-derived strain of Dichotomomyces cejpii (Figure 77). Emindole SB beta-mannoside (481) was identified as a $\mathrm{CB}_{2}$ antagonist $\left(K_{\mathrm{i}} 10.6 \mu \mathrm{M}\right)$, while 27-O-methylasporyzin $\mathrm{C}(\mathbf{4 8 2})$ was found to be a GPR18 (G-protein coupled receptor 18, $N$-arachidonyl glycine receptor) antagonist (IC50 $13.4 \mu \mathrm{M}$ ) [204].
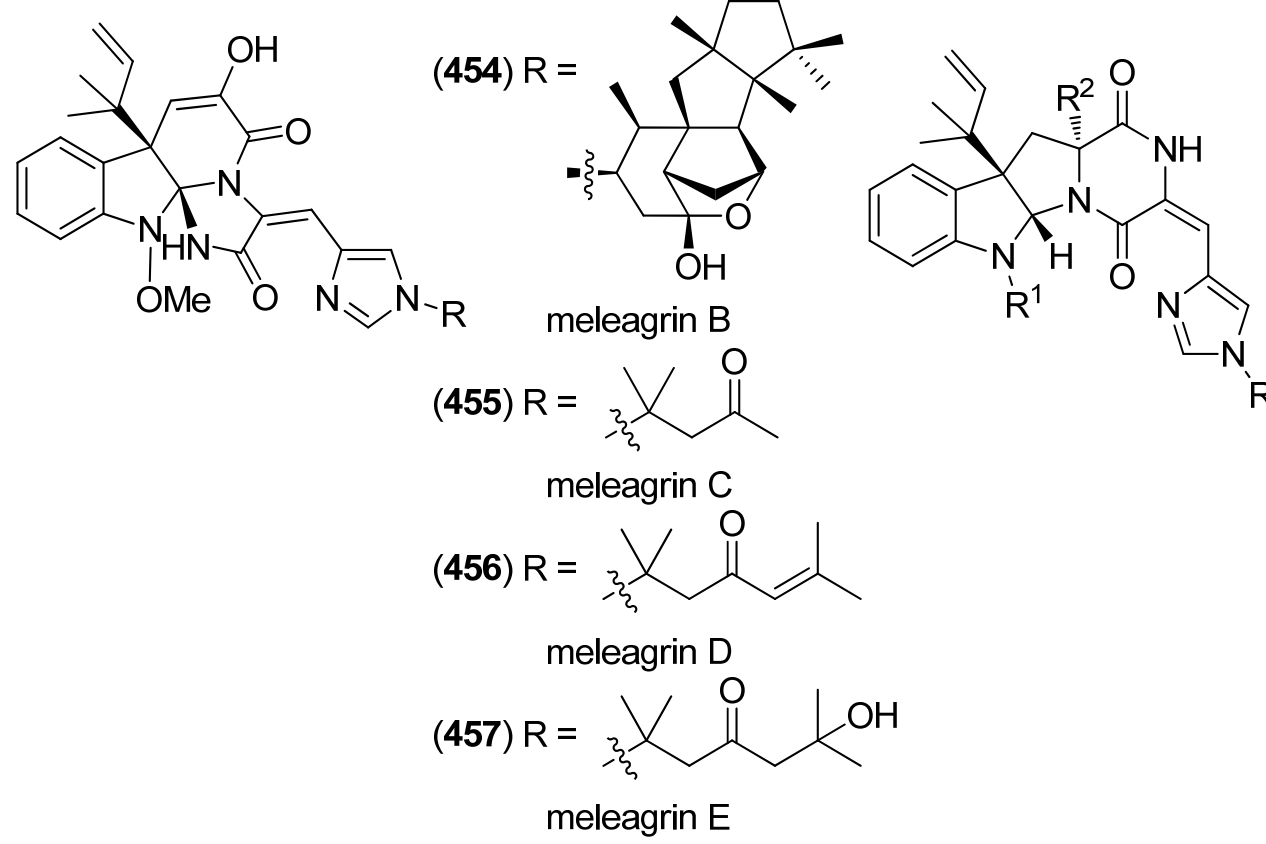
(458) $\mathrm{R}^{1}=\mathrm{OMe}, \mathrm{R}^{2,3}=\mathrm{H}$ roquefortine $F$

(459) $\mathrm{R}^{1}=\mathrm{H}, \mathrm{R}^{2}=\mathrm{OMe}$,

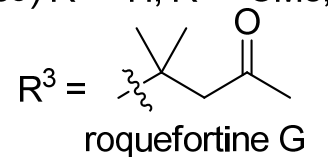

(460) $R^{1,2}=H$,

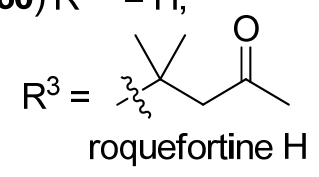

(461) $\mathrm{R}^{1,3}=\mathrm{H}, \mathrm{R}^{2}=\mathrm{OMe}$ roquefortine I

Figure 73. Maleagrins B-E and roquefortines F-I. 


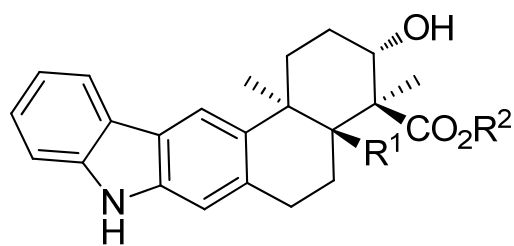

(462) $R^{1}=H, R^{2}=H$ xiamycin $A$ (463) $R^{1}=H, R^{2}=$ Me xiamycin A methyl ester (464) $R^{1}=\mathrm{OH}, \mathrm{R}^{2}=\mathrm{H}$ xiamycin $\mathrm{B}$<smiles>C[C@]12CC[C@@H](O)[C@H](C(=O)O)[C@H]1CCc1cc3[nH]c4ccccc4c3cc12</smiles><smiles>C[C@]12CC[C@@H](O)[C@@H](CO)[C@H]1CCc1cc3c(cc12)c1ccccc1n3N</smiles>

(466) dixiamycin A<smiles>C[C@H]1[C@@H](O)CC[C@]2(C)Oc3cc4c(cc3CC[C@@H]12)[nH]c1ccccc14</smiles>

(468) oxiamycin<smiles>C[C@]12CC[C@@H](O)[C@H](C(=O)O)[C@H]1CCC1=C2Cc2c([nH]c3ccccc23)C1</smiles>

(470) prexiamycin A<smiles>C=C1CC[C@@H]2[C@@H](CC[C@H](O)[C@@H]1C(=O)O)[C@@H]2Cc1c[nH]c2ccccc12</smiles>

(465) indosespene<smiles>C[C@]12CCC(O)[C@](C)(C(=O)O)[C@@H]1CCc1cc3c(cc12)c1ccccc1n3-n1c2ccccc2c2cc3c(cc21)CC[C@@]1(C)[C@]3(C)CC[C@@H](O)[C@@]1(C)C(=O)O</smiles>

(467) dixiamycin B<smiles>C[C@]12CC[C@@H](O)[C@H](C(=O)O)[C@H]1CCc1c2cc2c([nH]c3ccccc32)c1Cl</smiles>

(469) chloroxiamycin<smiles>C=C1CC[C@@H]2[C@@H](CC[C@H](O)C2(C)C)[C@H]1Cc1c[nH]c2ccccc12</smiles>

(471) preindosespene

Figure 74. Xiamycin A and its derivatives.

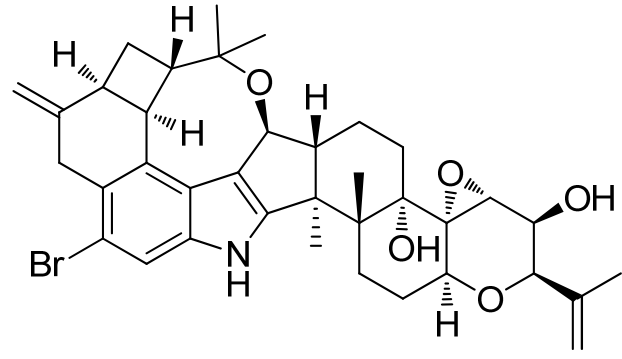

(472) 6-bromopenitrem B

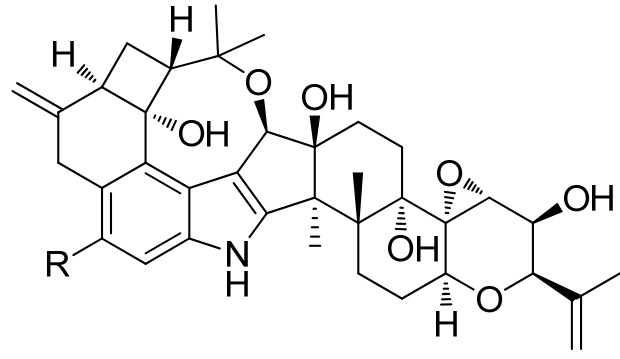

(473) $\mathrm{R}=\mathrm{Cl}$ 19-hydroxypenitrem A (474) $\mathrm{R}=\mathrm{Cl}$ 19-hydroxypenitrem E

Figure 75. Penitrem derivatives. 


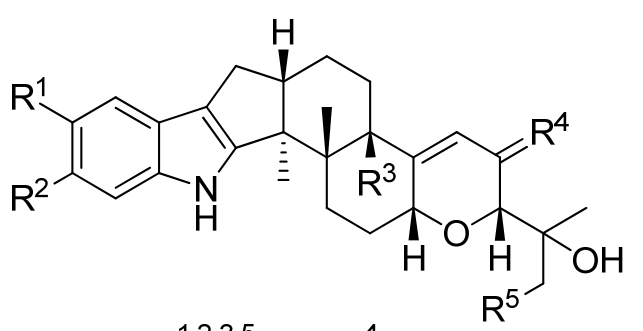

(475) $\mathrm{R}^{1,2,3,5}=\mathrm{H}, \mathrm{R}^{4}=\mathrm{H}_{2}$

(478) $\mathrm{R}^{1,2}=\mathrm{H}, \mathrm{R}^{3,5}=\mathrm{OH}, \mathrm{R}^{4}=\mathrm{O}$

(479) $R^{1,2}=-s^{3}$

$\mathrm{R}^{3}=\mathrm{OH}, \mathrm{R}^{4}=\mathrm{O}, \mathrm{R}^{5}=\mathrm{H}$

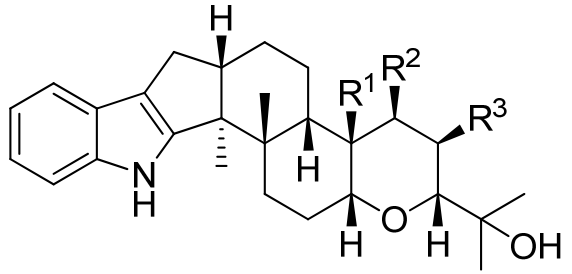

(476) $\mathrm{R}^{1}=\mathrm{CO}_{2} \mathrm{H}, \mathrm{R}^{2,3}=\mathrm{H}$

(477) $\mathrm{R}^{1,2,3}=\mathrm{OH}$

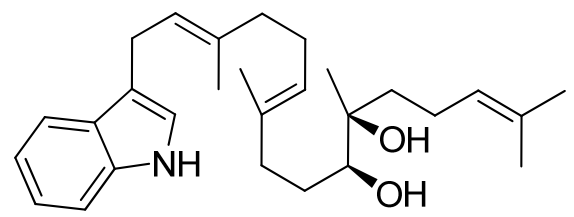

(480)

Figure 76. Metabolites from Penicillium camemberti.

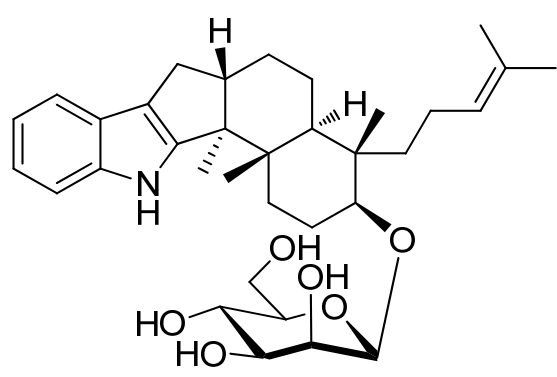

(481) emindole SB beta-mannoside

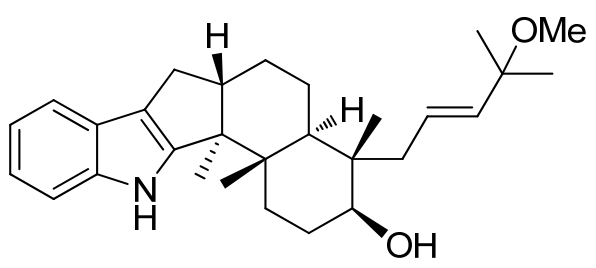

(482) 27-O-methylasporyzin C

Figure 77. Emindole SB beta-mannoside and 27-O-methylasporyzin $\mathrm{C}$.

Ergoline Alkaloids

Among the prenylated indole alkaloids, ergot alkaloids are a well-established group of natural products, known for their potent and manifold biological activities. Ergot alkaloids have been isolated from terrestrial sources exclusively until Pibocin A (483), the first representative of marine ergoline alkaloids, was obtained from extracts of the Far-Eastern ascidian Eudistoma sp. (Figure 78) [205]. Pibocins A (483) and B (484) [206] were found to show antimicrobial and cytotoxic effects against mouse Ehrlich carcinoma cells [205,206]. 2-(3,3-Dimethylprop-1-ene)-costaclavine (485) and 2-(3,3dimethylprop-1-ene)-epi-costaclavine (486) were isolated from the marine-derived fungus Aspergillus fumigatus, together with known clavine-type alkaloids costaclavine (487) and fumigaclavines A (488) [207] and C (489) [208]. Except of fumigaclavine A, all of them were found to show weak cytotoxicity against the mouse leukemia cell line P388 [209]. Additionally, fumigaclavine C (489) was found to induce apoptosis in MCF-7 breast cancer cells [210]. Ergosinine (490), which was isolated from the marine mollusc Pleurobranchus forskalii, is the first ergot peptide alkaloid (ergopeptine) found in marine life. The authors propose that ergot alkaloids may play a defensive or protective role in mollusks and other marine organisms [211]. 
<smiles>[R]n1c(Br)c2c3c(cccc31)[C@@]1(C)C[C@@H](C)CN(C)[C@H]1C2</smiles>

(483) $\mathrm{R}=\mathrm{H}$ pibocin $A$

(484) $R=$ OMe pibocin $B$<smiles>C[C@@H]1C[C@H]2Cc3c[nH]c4cccc(c34)C[C@H]2N(C)C1</smiles>

(487) costaclavine<smiles>C=CC(C)(C)c1[nH]c2cccc3c2c1C[C@H]1[C@H]3C[C@H](C)CN1C</smiles>

(485) $8 R$ (486) $8 S$<smiles>[R]c1[nH]c2cccc3c2c1C[C@@]1(C)[C@H](OC(C)=O)N(C)CC[C@H]31</smiles><smiles>CC(C)C[C@H]1C(=O)N2CCC[C@]2(O)[C@@]2(O)O[C@](C)(NC(=O)[C@@H]3C=C4c5cccc6[nH]cc(c56)C[C@H]4N(C)C3)C(=O)N12</smiles>

(490) ergosinine
(488) $\mathrm{R}=\mathrm{H}$ fumigaclavine $\mathrm{A}$

(489) $\mathrm{R}=$ fumigaclavine $\mathrm{C}$

Figure 78. Ergoline alkaloids.

\subsection{Bis- and Trisindoles}

Bis- and trisindole alkaloids are biosynthetically derived from two or three indole building blocks. They show diverse biological activities, as antiviral, antitumor, antibacterial and anti-inflammatory activities and are therefore promising chemical leads for drug development [212,213].

Trisindole alkaloid 1,1,3-tris(3-indolyl)butane (491) was isolated from a North Sea bacterium Vibrio parahaemolyticus Bio249, together with 3,3-bis(3-indolyl)butane-2-one (492), arundine (493), and 1,1,1-tris(3-indolyl)methane (494), which were isolated from a microorganism for the first time (Figure 79). Antibiotic or antifungal activities could not be evidenced [214].

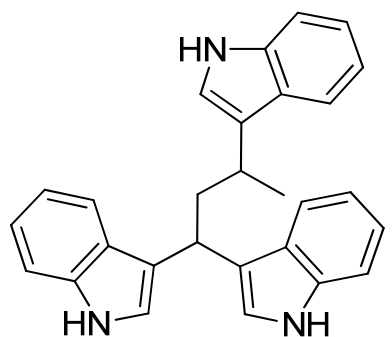

(491)

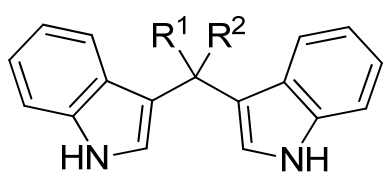

(492) $R^{1}=A c, R^{2}=M e$

(493) $R^{1,2}=H$ arundine

(494) $\mathrm{R}^{1}=\mathrm{H}, \mathrm{R}^{2}=1 H$-indol-3-yl-

Figure 79. Bis- and trisindoles.

Dendridine A (495) was isolated from the marine sponge Dictyodendrilla sp. and showed antibacterial activity against Bacillus subtilis and Micrococcus luteus (MIC 8.3 and $4.2 \mu \mathrm{g} / \mathrm{mL}$ ) as well as antifungal activity against Cryptococcus neoformans (MIC $8.3 \mu \mathrm{g} / \mathrm{mL}$ ) and weak cytotoxic effects against L1210 tumor cells ( $\mathrm{IC}_{50} 32.5 \mu \mathrm{g} / \mathrm{mL}$ ) (Figure 80) [215]. Hyrtinadine A (496) was obtained from an Okinawan marine sponge Hyrtios sp. (SS-1127). It displayed cytotoxic activities against 
murine leukemia L1210 cells and human epidermoid carcinoma KB cells ( $\mathrm{IC}_{50} 1$ and $3 \mu \mathrm{g} / \mathrm{mL}$, respectively) [216].<smiles>NCCc1c[nH]c2c(O)cc(Br)c(-c3c(Br)cc(O)c4[nH]cc(CCN)c34)c12</smiles>

(495) Dendridine A<smiles>Oc1ccc2c(-c3cnc(-c4c[nH]c5cc(O)ccc45)cn3)c[nH]c2c1</smiles>

(496) hyrtinadine A

Figure 80. Dendridine A and hyrtinadine A.

Polybrominated bisindoles 3,3'-bis(2'-methylsulfinyl-2-methylthio-4,6,4',6'-tetrabromo)indole (497) and 3,3'-bis(4,6-dibromo-2-methylsulfinyl)indole (498) were isolated from the Formosan red alga Laurencia brongniartii (Figure 81). Bisindoles 497 and 498 displayed cytotoxicity against HT-29 and P388 cell lines, respectively [40]. 2,2',5,5',6,6'-Hexabromo-3,3'-bis-1H-indole (499) was isolated from the marine red alga Laurencia similis [44].<smiles>[R]c1[nH]c2cc(Br)cc(Br)c2c1-c1c([R])[nH]c2cc(Br)cc(Br)c12</smiles>

(497) $\mathrm{R}^{1}=\mathrm{SMe}, \mathrm{R}^{2}=\mathrm{S}(\mathrm{O}) \mathrm{Me}$ (498) $R^{1}, R^{2}=S(O) M e$<smiles>Brc1cc2[nH]c(Br)c(-c3c(Br)[nH]c4cc(Br)c(Br)cc34)c2cc1Br</smiles>

(499) 2,2',5,5',6,6'-hexabromo-3,3'-bi-1H-indole

Figure 81. Polybrominated bisindoles.

Arsindolines A (500) and B (501) were isolated from a marine-derived bacterium strain Aeromonas sp. CB101 (Figure 82). Arsindoline B (501) showed antitumor activity against cell line A549 (IC50 $22.6 \mu \mathrm{M})$ [217]. Metagenetriindole A (502) and metagenediindole A (503) were obtained from a deep-sea sediment derived Escherichia coli strain and exhibited moderate cytotoxic activity against CNE2, Bel7402 and HT1080 cancer cell lines (IC50 34.25-50.55 $\mu \mathrm{g} / \mathrm{mL}$ ) [218].

The yellow pigment halichrome A (504) was isolated from a metagenomic library derived from the marine sponge Halichondria okadai and showed cytotoxicity against B16 melanoma cells (Figure 83) [219]. Scalaridine A (505), together with 5-hydroxyindole alkaloids $\mathbf{1 5 0}$ and 151, was isolated from the marine sponge Scalarispongia sp. collected near Dokdo Island. It is the first bisindole alkaloid with a pyridine linker and exhibits cytotoxic activity against human leukemia cells K562 (IC50 $39.5 \mu \mathrm{g} / \mathrm{mL})$ [97]. 
<smiles>c1ccc2c(C(c3c[nH]c4ccccc34)c3c[nH]c4ccccc34)ccnc2c1</smiles>

(500) arsindoline A<smiles>O=C(c1c[nH]c2ccccc12)C(c1c[nH]c2ccccc12)c1c[nH]c2ccccc12</smiles>

(502) metagenetriindole A<smiles>CCCC(=O)OCC(c1c[nH]c2ccccc12)c1c[nH]c2ccccc12</smiles>

(501) arsindoline B<smiles>CC(c1c[nH]c2ccccc12)C1Nc2ccccc2C1=O</smiles>

(503) metagenediindole A

Figure 82. Arsindolines A, B, metagenetriindole A and metagenediindole A.<smiles>CCC1(c2cc3ccccc3[nH]2)Nc2ccccc2C1=O</smiles>

(504) halichrome $A$<smiles>Oc1ccc2[nH]cc(-c3cncc(-c4c[nH]c5ccc(O)cc45)c3)c2c1</smiles>

(505) scalaridine A

Figure 83. Halichrome A and scalaridine A.

Bisindolic 1,2-di(1H-indol-3-yl)ethane (506, Figure 84) was isolated from the marine bacterium Pantoea agglomerans P20-14, together with monoindoles 87 and 88 (see Figure 21) [73].<smiles>c1ccc2c(CCc3c[nH]c4ccccc34)c[nH]c2c1</smiles>

(506) 1,2-di(1H-indol-3-yl)ethane

Figure 84. 1,2-Di(1H-indol-3-yl)ethane.

Shewanellines A-C (507, 508 and 139) were isolated from the deep-sea bacterium Shewanella piezotolerans WP3 (Figure 85). Since shewanelline C does not belong to the bisindoles, it is discussed with the simple indole alkaloids (see Figure 34) [89]. 
<smiles>CO[C@H]1C(=O)Nc2ccccc21</smiles>

(507) 3 S shewanelline A

(508) $3 R$ shewanelline $B$

Figure 85. Shewanellines A and B.

2-(2-(3-Hydroxy-1-(1H-indol-3-yl)-2-methoxypropyl)-1H-indol-3-yl) acetic acid (509) and 3-(3-(2-hydroxyethyl)-1H-indol-2-yl)-3-(1H-indol-3-yl)propane-1,2-diol) (510) were isolated from the marine actinomycete Rubrobacter radiotolerans (Figure 86). Both showed AchE inhibitory activity but no significant cytotoxic effects [220].<smiles>COC(CO)[C@H](c1[nH]c2ccccc2c1CC(=O)O)c1c[nH]c2ccccc12</smiles>

(509)<smiles>OCCc1c([C@@H](c2c[nH]c3ccccc23)C(O)CO)[nH]c2ccccc12</smiles>

(510)

Figure 86. 2-(2-(3-Hydroxy-1-(1H-indol-3-yl)-2-methoxypropyl)-1H-indol-3-yl) acetic acid and 3-(3-(2-hydroxyethyl)-1H-indol-2-yl)-3-(1H-indol-3-yl)propane-1,2-diol).

Echinosulfonic acid D (511) was isolated from the New-Caledonian sponge Psammoclemma sp. and showed cytotoxic effects towards KB cells (IC50 $2 \mu \mathrm{g} / \mathrm{mL}$ ) (Figure 87) [221].<smiles>COC(=O)n1cc(C(c2c[nH]c3cc(Br)ccc23)S(=O)(=O)O)c2ccc(Br)cc21</smiles>

Figure 87. Echinosulfonic acid D.

Hyrtiazepine (512) was isolated from Hyrtios erectus (Figure 88) [35]. 


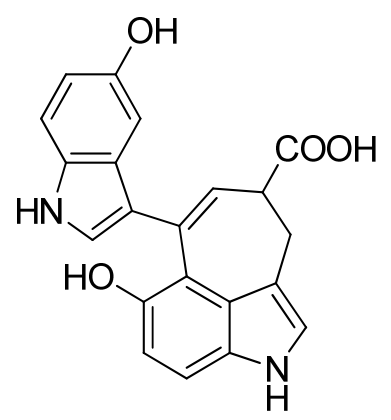

(512) hyrtiazepine

Figure 88. Hyrtiazepine.

Leptosins O-S (513-517) were isolated from a strain of Leptosphaeria sp., originated from the marine alga Sargassum tortile (Figure 89). Leptosins O (513) and P (514) exhibited cytotoxic activity against P388 cells [222].

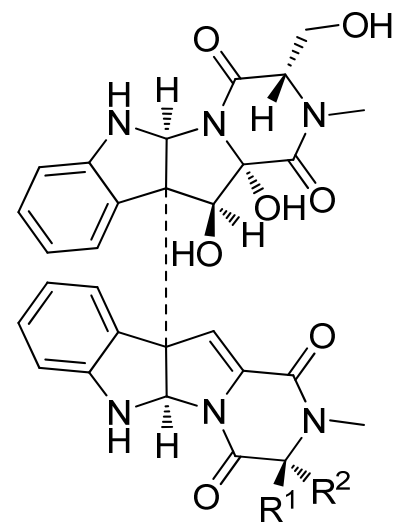

(513) $\mathrm{R}^{1}=\mathrm{S}_{2} \mathrm{Me}, \mathrm{R}^{2}=\mathrm{CHMe}_{2}$ leptosin $\mathrm{O}$

(514) $R^{1}=\mathrm{CHMe}_{2}, R^{2}=\mathrm{S}_{2} \mathrm{Me}$ leptosin $P$

(515) $R^{1}=S M e, R^{2}=\mathrm{CHMe}_{2}$ leptosin $Q$

(516) $R^{1}=\mathrm{CHMe}_{2}, \mathrm{R}^{2}=\mathrm{SMe}$ leptosin $\mathrm{R}$

(517) $\mathrm{R}^{1}=\mathrm{NH}_{2}, \mathrm{R}^{2}=\mathrm{CHMe}_{2}$ leptosin $\mathrm{S}$

Figure 89. Leptosins $\mathrm{O}-\mathrm{S}$.

Gliocladins A-C (518-520) and glioperazine (521) were isolated from a sea hare-derived strain of Gliocladium sp. (Figure 90). Gliocladin C (520) displayed cytotoxic activity against P388 cells [223].

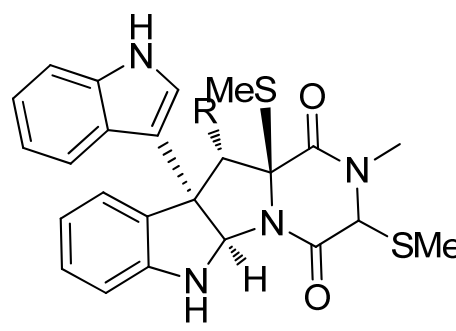

(518) $\mathrm{R}=\mathrm{OH}$ gliocladin $\mathrm{A}$ (519) $\mathrm{R}=\mathrm{H}$ gliocladin $\mathrm{B}$<smiles>CN1C(=O)C(=O)N2C(=C[C@@]3(c4c[nH]c5ccccc45)c4ccccc4N[C@@H]23)C1=O</smiles>

(520) gliocladin C<smiles>CC(O)[C@]1(S(C)(C)C)NC(=O)[C@](C)(Cc2c[nH]c3ccccc23)NC1=O</smiles>

(521) glioperazine

Figure 90. Gliocladins A-C and glioperazine.

ZHD-0501 (522), a staurosporine analog, was isolated from the marine-derived Actinomadura sp. 007 and displayed cytotoxic activity towards the cancer cell lines A549, BEL-7402, HL-60, P388 and tsFT210 (Figure 91) [224]. $\mathrm{N}$-Carboxamido-staurosporine (523) was isolated from the marine-derived Streptomyces sp. QD518. It was found to have antibacterial, as well as potent and selective cytotoxic, 
activity [225]. 7-Oxo-3,8,9-trihydroxystaurosporine (524) and 7-oxo-8,9-dihydroxy-4'- $N$ demethylstaurosporine (525) were isolated from the marine ascidian Cystodytes solitus. As is common for this compound class, both compounds exhibited cytotoxicity towards A549, HT-29 and MDA-MB-231 cell lines (GI50 17.5-90 nM) [226]. 2-Hydroxy-7-oxostaurosporine (526) and 3-hydroxy-7oxostaurosporine (527) were isolated from the Brazilian tunicate Eudistoma vannamei and showed cytotoxicity against the human cancer cell lines HL-60, MOLT-4, Jurkat, K562, HCT-8, SF-295 and MDA-MB-435 (IC50 10.33-144.47 nM) [227].

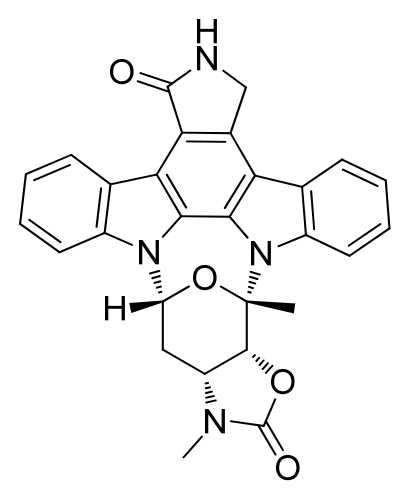

(522) ZHD-0501

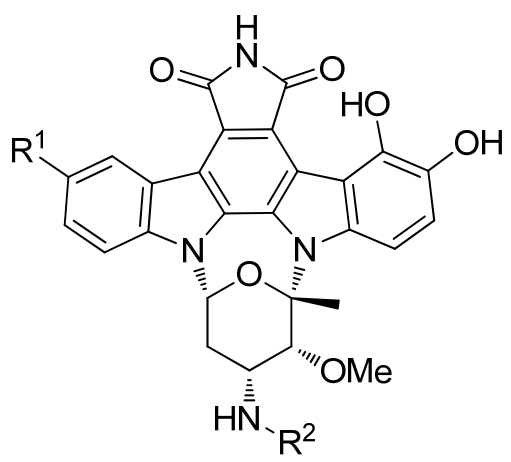

(524) $\mathrm{R}^{1}=\mathrm{OH}, \mathrm{R}^{2}=\mathrm{Me}$

(525) $R^{1,2}=H$

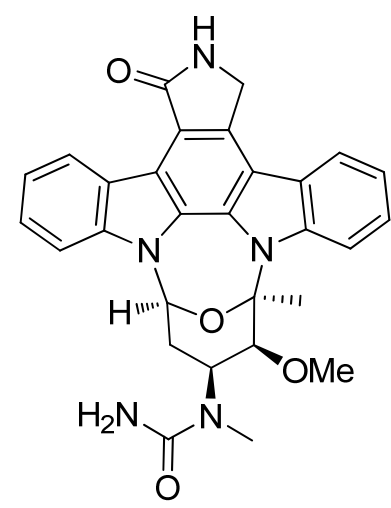

(523) $\mathrm{N}$-carboxamido-staurosporine<smiles></smiles>

(526) $\mathrm{R}^{1}=\mathrm{OH}, \mathrm{R}^{2}=\mathrm{H}$

(527) $\mathrm{R}^{1}=\mathrm{H}, \mathrm{R}^{2}=\mathrm{OH}$

Figure 91. Staurosporine derivatives.

Analysis of the marine-derived actinomycetes strain Streptomyces sp. FMA led to the discovery of streptocarbazoles A (528) and B (529) (Figure 92). Streptocarbazole A (528) has cytotoxic activity towards L-60, A549, P388 and HeLa cell lines (IC $\mathrm{I}_{50}$ 1.4, 5.0, 18.9, and $34.5 \mu \mathrm{M}$, respectively) and arrests the cell cycle of HeLa cells in the $\mathrm{G}_{2} / \mathrm{M}$ phase at a concentration of $10 \mu \mathrm{M}$ [228]. Fradcarbazoles A-C (530-532) were obtained from a mutant strain of the marine-derived actinomycete Streptomyces fradiae 007M135. They exhibited strong cytotoxic effects towards HL-60, K562, A549 and BEL-7402 cell lines (IC50 $0.001-4.58 \mu \mathrm{M}$ ) and were found to be potent kinase PKC- $\alpha$ inhibitors (IC50 $0.16-4.27 \mu \mathrm{M})$ [229].

Dictyodendrins A-E (533-537) were obtained from the Japanese marine sponge Dictyodendrilla verongiformis and exhibited telomerase inhibitory activity (Figure 93) [230]. Dictyodendrins F-J (538-542) were isolated from a southern Australian marine sponge Ianthella sp. (CMB-01245). Dictyodendrins F (538) and H-J (540-542) exhibited protease $\beta$-secretase (BACE) inhibitory activity 
and dictyodendrins F-I (538-541) have cytotoxic activity against human colon cancer cell line SW620 and the P-glycoprotein over-expressing SW620 Ad300 [231].<smiles>[R]CC1=C(OC)[C@@]2(OC)C[C@H](O1)n1c3ccccc3c3c4c(c5c6ccccc6n2c5c31)C(=O)NC4</smiles>

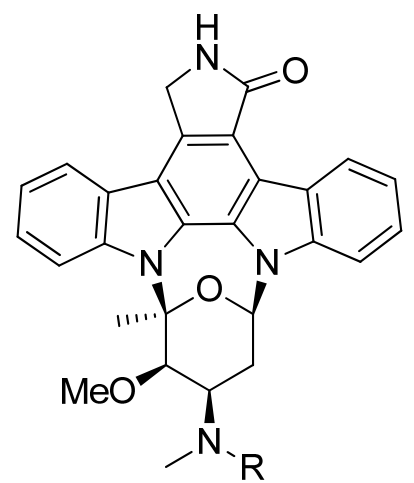

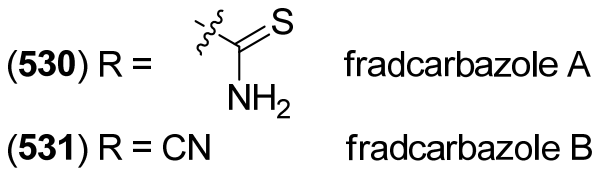<smiles>[13CH3]</smiles><smiles>COc1cc(-c2c[nH]c3ccccc23)sc1C(C)(C)C</smiles>

(528) $\mathrm{R}=\mathrm{OH}$ streptocarbazole $\mathrm{A}$

(529) $\mathrm{R}=\mathrm{H}$ streptocarbazole $B$

Figure 92. Streptocarbazoles A and B and fradcarbazoles A-C.<smiles>COC(=O)[C@H](c1ccc(O)cc1)c1c(-c2ccc(O)cc2)c2c3c([nH]c4c(OC(N)=O)cccc43)c(O)c(-c3ccc(O)cc3)c2n1CCc1ccc(O)cc1</smiles>

(533) dictyodendrin A<smiles></smiles><smiles>CS(=O)(=O)Oc1cccc2c1[nH]c1c(O)c(-c3ccc(O)cc3)c3c(c(-c4ccc(O)cc4)c(C(=O)c4ccc(O)cc4)n3CCc3ccc(O)cc3)c12</smiles>

(534) dictyodendrin B<smiles>[R1]Oc1cccc2c3c([nH]c12)C(=O)C(c1ccc(O)cc1)=C1C3=C(c2ccc(O)cc2)C(=O)N1CCc1ccc(O)c([R])c1</smiles><smiles>[R6]c1ccc(CCN2C(=O)C(c3ccc(O)cc3)=C3C2=C(c2ccc(O)cc2)C(=O)c2[nH]c4c(OS(N)(=O)=O)cccc4c23)cc1</smiles>

(535) $\mathrm{R}=\mathrm{H}$ dictyodendrin $\mathrm{C}$

(536) $\mathrm{R}=\mathrm{SO}_{3} \mathrm{Na}$ dictyodendrin $\mathrm{D}$<smiles>O=C(C(=O)c1[nH]c2c(O)cccc2c1C1=C(c2ccc(O)cc2)C(=O)N(CCc2ccc(O)cc2)C1=O)c1ccc(O)cc1</smiles>

(542) dictyodendrin J

(537) dictyodendrin $\mathrm{E}$

(538) $\mathrm{R}^{1,2}=\mathrm{H}$ dictyodendrin $\mathrm{F}$

(539) $R^{1}=M e, R^{2}=H$ dictyodendrin $G$

(540) $\mathrm{R}^{1}=\mathrm{H}, \mathrm{R}^{2}=\mathrm{Br}$ dictyodendrin $\mathrm{H}$

(541) $R^{1}=H, R^{2}=$ I dictyodendrin I

Figure 93. Dictyodendrins A-J. 
Tubastrindoles A-C (543-545) were isolated from a stony coral, Tubastraea sp. (Figure 94) [232]. Tubastrindole B (544) turned out to be a potent and selective $\alpha 1$ GlyR (glycine-gated chloride channel receptor) antagonist ( $\left.\mathrm{IC}_{50} 25.9 \mu \mathrm{M}\right)$ [233]. Tubastrindoles D-H (546-550) were obtained from Tubastraea aurea (Odomari, Kagoshima, Japan) [234]. Bisindole alkaloid 551 was also isolated from Tubastraea sp. and exhibited antiplasmodial activity [235].

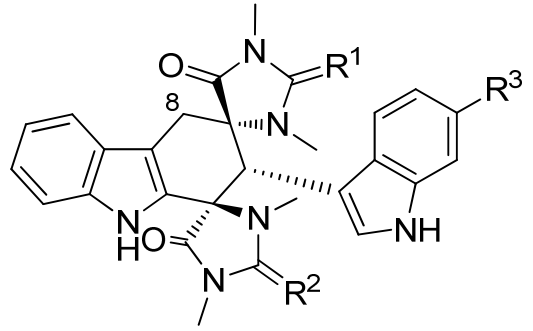

(543) $\mathrm{R}^{1,2}=\mathrm{NH}, \mathrm{R}^{3}=\mathrm{Br}$ tubastrindole $\mathrm{A}$

(544) $\mathrm{R}^{1,2}=\mathrm{NH}, \mathrm{R}^{3}=\mathrm{H}$ tubastrindole $\mathrm{B}$

(545) $\mathrm{R}^{1}=\mathrm{NH}, \mathrm{R}^{2}=\mathrm{O}, \mathrm{R}^{3}=\mathrm{H}$ tubastrindole $\mathrm{C}$

(546) $\mathrm{R}^{1}=\mathrm{NH}, \mathrm{R}^{2}=\mathrm{O}, \mathrm{R}^{3}=\mathrm{Br}$ tubastrindole $\mathrm{D}$

(547) $\mathrm{R}^{1}=\mathrm{NH}, \mathrm{R}^{2}=\mathrm{O}, \mathrm{R}^{3}=\mathrm{H}, 8 \alpha=\mathrm{OH}$ tubastrindole $\mathrm{E}$

(548) $\mathrm{R}^{1}=\mathrm{O}, \mathrm{R}^{2}=\mathrm{NH}, \mathrm{R}^{3}=\mathrm{Br}$ tubastrindole $\mathrm{F}$

(549) $\mathrm{R}^{1}=\mathrm{O}, \mathrm{R}^{2}=\mathrm{NH}, \mathrm{R}^{3}=\mathrm{H}$ tubastrindole $\mathrm{G}$

(550) $\mathrm{R}^{1,2}=\mathrm{O}, \mathrm{R}^{3}=\mathrm{H}$ tubastrindole $\mathrm{H}$

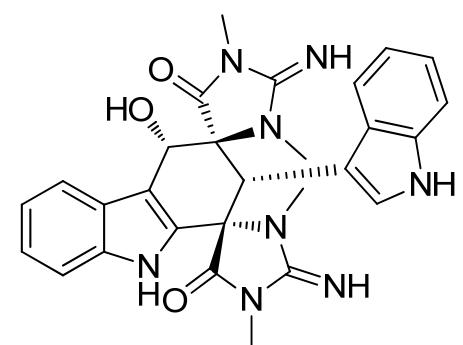

(551)

Figure 94. Tubastrindoles A-H.

Rostratins A-D (552-555) were obtained from a marine-derived strain of the fungus Exserohilum rostratum (Drechsler) and exhibited cytotoxic activity against human colon carcinoma HCT-116 (IC50 8.5, 1.9, 0.76 and $16.5 \mu \mathrm{g} / \mathrm{mL}$, respectively) (Figure 95) [236].

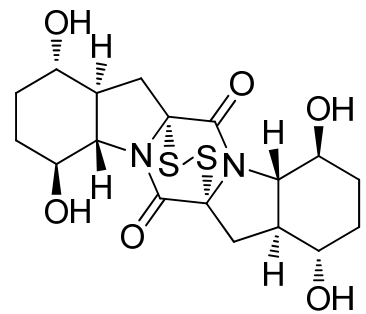

(552) rostratin $A$

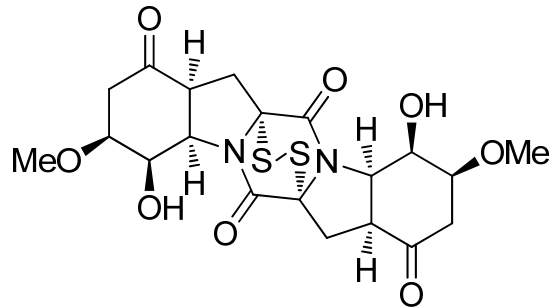

(554) rostratin C

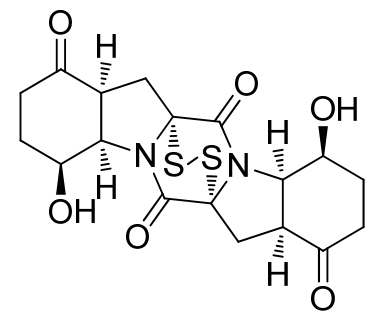

(553) rostratin B

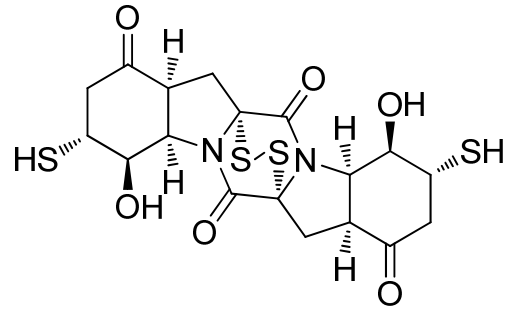

(555) rostratin D

Figure 95. Rostratins A-D.

Diketopiperazine dimer $\mathbf{5 5 6}$ was isolated from a marine-derived isolate of Aspergillus niger (Figure 96) [237]. 


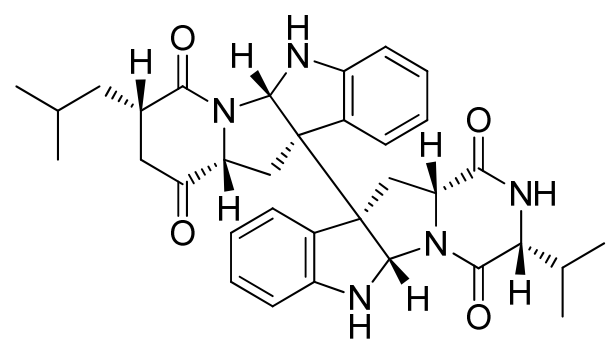

(556) diketopiperazine dimer

Figure 96. Diketopiperazine dimer.

(R)-6"-Debromohamacanthin B (556) was isolated from the marine sponge Spongosorites $\mathrm{sp}$. (556) exhibited low inhibitory activity against $S$. aureus sortase A (SrtA) (Figure 97) [238]. Nine new bisindole alkaloids were isolated from the same organism, five of them belonging to the hamacanthin $(\mathbf{5 5 7}-\mathbf{5 6 0}, \mathbf{5 6 2})$ and four to the topsentin class $(\mathbf{5 6 3}-\mathbf{5 6 6})$. The structure of spongotine B (565) was erroneously reported as $(S)-6 "$-debromohamacanthin B in the earlier report of Bao et al. [239,240]. They partially exhibited cytotoxic activity against the human tumor cell lines A549, SK-OV-3, SK-MEL-2, XF498 and HCT-15, as well as weak antibacterial activity against several methicillin-resistant strains $[239,240]$.<smiles>[R]c1ccc2c(C3=NC(c4c[nH]c5cc([R])ccc45)NC3=O)c[nH]c2c1</smiles><smiles>[R]c1ccc2c(C3=N[C@H](c4c[nH]c5cc([R])ccc45)CNC3=O)c[nH]c2c1</smiles>

(557) $\mathrm{R}^{1}=\mathrm{Br}, \mathrm{R}^{2}=\mathrm{H}, 6 \mathrm{R}$ $(R)-6$ "-debromohamacanthin $\mathrm{A}$ (558) $\mathrm{R}^{1}=\mathrm{H}, \mathrm{R}^{2}=\mathrm{Br}, 6 \mathrm{R}$ (R)-6'-debromohamacanthin $A$ (559) $\mathrm{R}^{1,2}=\mathrm{H}, 6 \mathrm{~S}$

(560) $\mathrm{R}^{1,2}=\mathrm{H}, 5 \mathrm{R}$ $(R)-6$ ',6"-didebromohamacanthin B (561) $\mathrm{R}^{1}=\mathrm{Br}, \mathrm{R}^{2}=\mathrm{H}, 5 \mathrm{R}$ $(R)-6$ "-debromohamacanthin $\mathrm{B}$

(S)-6',6"-didebromohamacanthin A

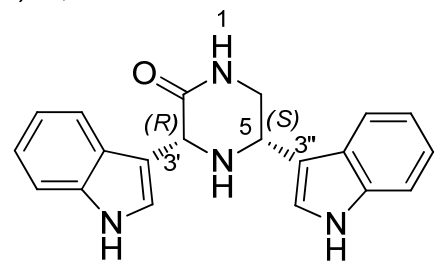

(562) (3S,5R)-6',6"-didebromo-3,4dihydrohamacanthin $B$<smiles>[R]c1ccc2c(C(=O)C3=N[C@H](c4c[nH]c5cc([R])ccc45)CN3)c[nH]c2c1</smiles>

(564) $R^{1}=B r, R^{2}=H$, spongotine $A$

(565) $R^{1}=H, R^{2}=B r$, spongotine $B$

(566) $\mathrm{R}^{1,2}=\mathrm{Br}$, spongotine $\mathrm{C}$<smiles>O=C(c1ncc(-c2c[nH]c3cc(Br)ccc23)[nH]1)c1c[nH]c2cc(Br)ccc12</smiles>

(563) dibromodeoxytopsentin 
Figure 97. Hamacanthin and topsentin derivatives.

6"-Debromohamacanthin A (557) revealed significant antibacterial activity against Gram-positive and Gram-negative bacteria including MRSA (Methicillin-resistant Staphylococcus aureus), as well as antifungal activity [241]. It was also found out to target the VEGFR2 (vascular endothelial growth factor receptor 2)-mediated PI3K/AKT/mTOR signaling pathway and, thus, effectively inhibit angiogenesis [242]. 6"-Debromohamacanthin A (557), 6"-debromohamacanthin B (561) and spongotine A (564) showed antitumor activity against AGS and L1210 cancer cell lines [241]. Figure 98 shows a hypothetical biosynthesis of topsentins and hamacanthins from monomeric indole pyruvic acid derivatives [56].

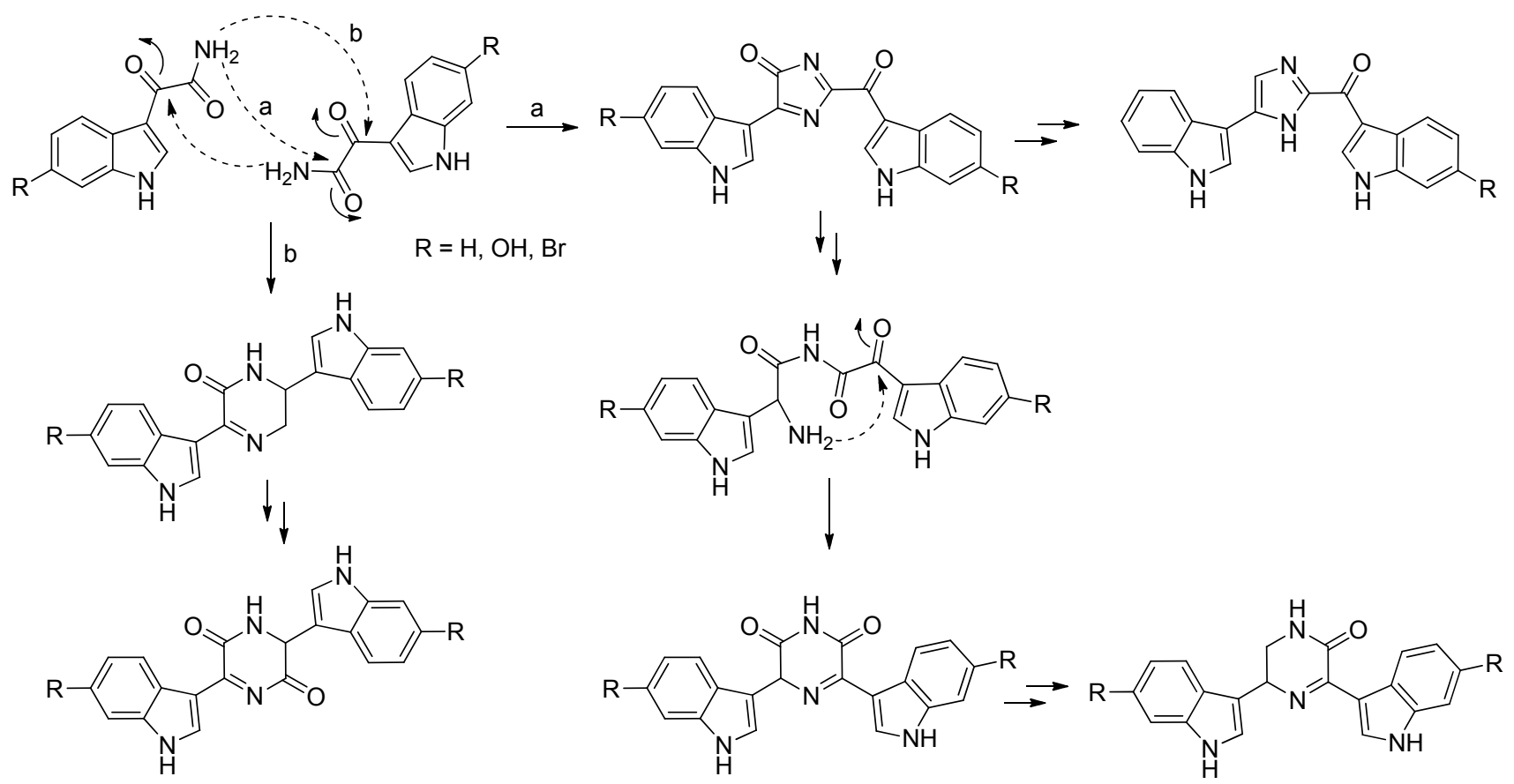

Figure 98. Hypothetical biosynthesis of topsentins and hamacanthins [56].

Two unprecedented brominated spiro-trisindole alkaloids, similisines A (567) and B (568), were isolated from L. similis (Figure 99) [243].<smiles>C[C@H]1C(=O)[C@]2(C(=O)Nc3cc(Br)c(Br)cc32)c2[nH]c3cc(Br)c(Br)cc3c2[C@@H]1c1c(Br)[nH]c2cc(Br)c(Br)cc12</smiles>

(567) similisine A

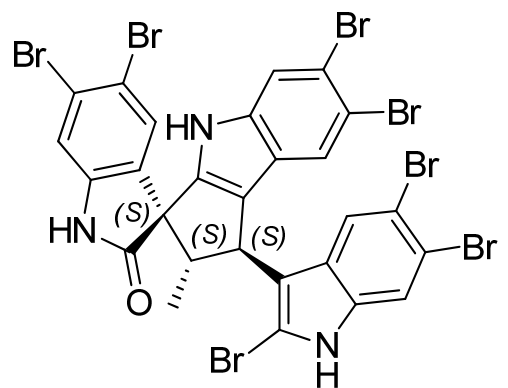

(568) similisine B

Figure 99. Similisines A and B. 
Dictazolines A-E (569-573) and dictazoles A (574) and B (575) were obtained from the marine sponge Smenospongia cerebriformis (Figure 100). Dictazoles are possible biosynthetic precursors of the dictazolines, and may be transformed to the latter via vinyl cyclobutane rearrangement [244,245].

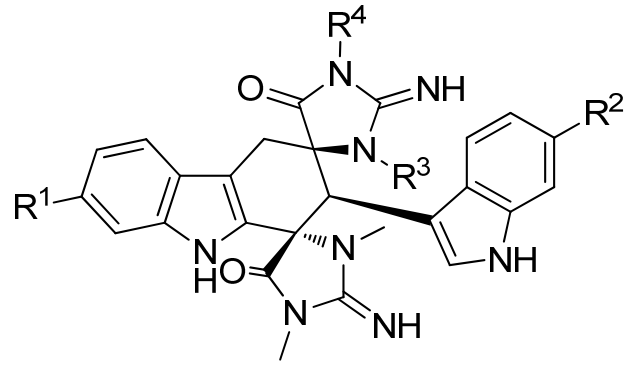

(569) $\mathrm{R}^{1,2}=\mathrm{Br}, \mathrm{R}^{3,4}=$ Me dictazoline $\mathrm{A}$

(570) $R^{1,2}=B r, R^{3,4}=H$ dictazoline $B$

(571) $\mathrm{R}^{1}=\mathrm{Br}, \mathrm{R}^{2,3,4}=\mathrm{H}$ dictazoline $\mathrm{C}$

(572) $R^{1}=B r, R^{2,3}=H, R^{4}=$ Me dictazoline $D$

(573) $R^{1,2,3}=H, R^{4}=$ Me dictazoline $E$

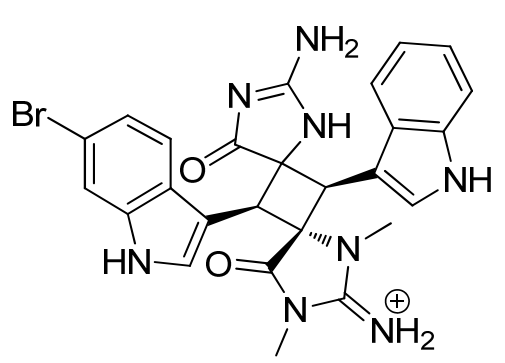

(574) dictazole A

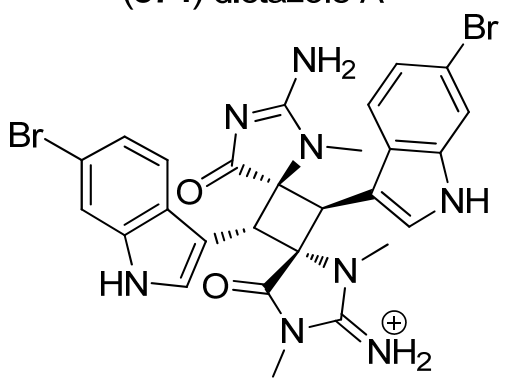

(575) dictazole B

Figure 100. Dictazolines A-E and dictazoles A and B.

Eusynstyelamides A-C (576-578) were isolated from the Great Barrier Reef ascidian Eusynstyela latericius [246] whereas eusynstyelamides D-F (579-581) [247] were isolated from the extract of the Arctic bryozoan Tegella cf. spitzbergensis (Figure 101). Eusynstyelamide B (577) displays cytotoxic activity against MDA-MB-231 cells and was identified as a potent cell cycle inhibitor [248]. It also inhibits proliferation of $\mathrm{LNCaP}$ cells in $\mathrm{G} 2$ phase and is indicated to be a topoisomerase II inhibitor in LNCaP cells [249]. Eusynstyelamides A-C (576-578) exhibit inhibitory activity against neuronal nitric oxide synthase ( $n$ NOS, $\mathrm{IC}_{50} 41.7,4.3$ and $5.8 \mu \mathrm{M}$, respectively), furthermore antimicrobial activity (Staphylococcus aureus, Escherichia coli, Pseudomonas aeruginosa, Corynebacterium glutamicum, and MRSA have been tested, $\mathrm{IC}_{50}$ between 6.25 and $>50 \mu \mathrm{M}$ ) was reported for eusynstyelamides B (577) and D-F (579-581). Eusynstyelamides A (576) and B (577) show weak inhibitory activity towards pyruvate phosphate dikinase (PPDK, IC 5019 and $20 \mathrm{mM}$, respectively) [246,247].

Naseseazines A (582) and B (583) were obtained from Streptomyces sp. (CMB-MQ030) isolated from a Fijian marine sediment (Figure 102). They displayed neither antibacterial, nor antifungal or cytotoxic activities [250].

Plectosphaeroic acids A-C (584-586) were obtained from the marine fungus Plectosphaerella cucumerina and identified to have inhibitory activity on indoleamine 2,3-dioxygenase (IDO, IC 50 about $2 \mu \mathrm{M}$ ) (Figure 103) [251].

Extraction of the marine sponge Clathria (Thalysias) araiosa, collected from Vanuatu, led to the identification of the nitrogen-rich and highly polar tris-bromoindole cyclic guanidine alkaloids araiosamines A-D (587-590) (Figure 104) [252]. 
<smiles>N=C(N)NCCCCNC(=O)[C@@]1(O)[C@H](Cc2c[nH]c3cc(Br)ccc23)[C@H](Cc2c[nH]c3cc(Br)ccc23)C(=O)N1CCCCNC(=N)N</smiles>

(576) eusynstyelamide A<smiles>N=C(N)NCCCCNC(=O)[C@]1(O)[C@H](Cc2c[nH]c3cc(Br)ccc23)[C@H](O)[C@H](Cc2c[nH]c3cc(Br)ccc23)C(=O)N1CCCCNC(=N)N</smiles>

(578) eusynstyelamide C<smiles>N=C(N)NCCCCN1C(=O)[C@H](O)[C@H](Cc2c[nH]c3cc(Br)ccc23)[C@@]1(O)C(=O)NCCCCN</smiles>

(580) eusynstyelamide E<smiles>N=C(N)NCCCCNC(=O)[C@]1(O)[C@H](Cc2c[nH]c3cc(Br)ccc23)[C@@H](O)C(=O)N1CCCCNC(=N)N</smiles>

(577) eusynstyelamide B<smiles>NCCCCNC(=O)[C@]1(O)[C@H](Cc2c[nH]c3cc(Br)ccc23)[C@@H](O)C(=O)N1CCCCN</smiles>

(579) eusynstyelamide D<smiles>N=C(N)NCCCCNC(=O)[C@]1(O)[C@H](Cc2c[nH]c3cc(Br)ccc23)[C@H](O)[C@H](Cc2c[nH]c3cc(Br)ccc23)C(=O)N1CCCCN</smiles>

(581) eusynstyelamide F

Figure 101. Eusynstyelamides A-F.

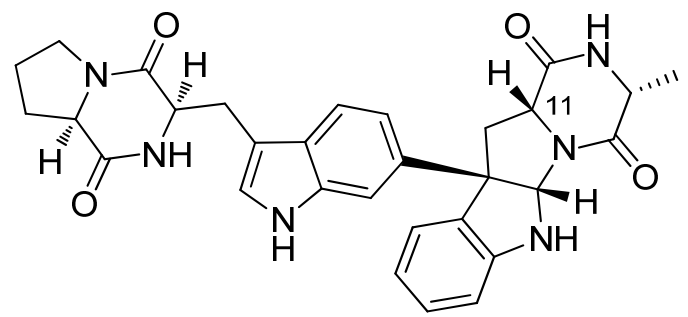

(582) naseseazine A

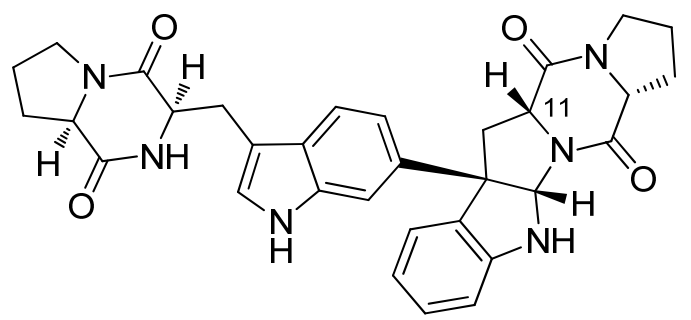

(583) naseseazine B

Figure 102. Naseseazines A and B. 


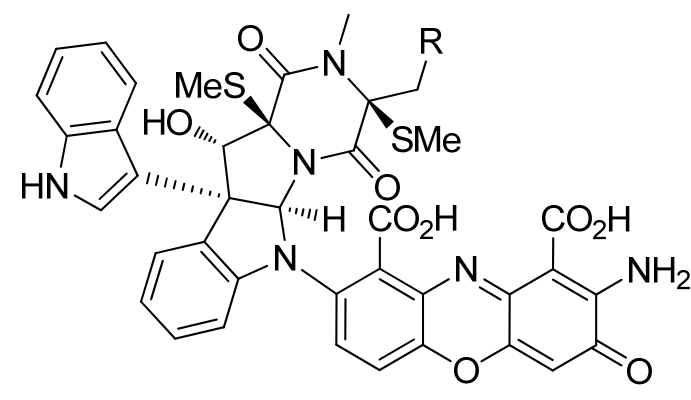

(584) $\mathrm{R}=\mathrm{OH}$ plectosphaeroic acid $\mathrm{A}$ (585) $\mathrm{R}=\mathrm{H}$ plectosphaeroic acid $\mathrm{B}$

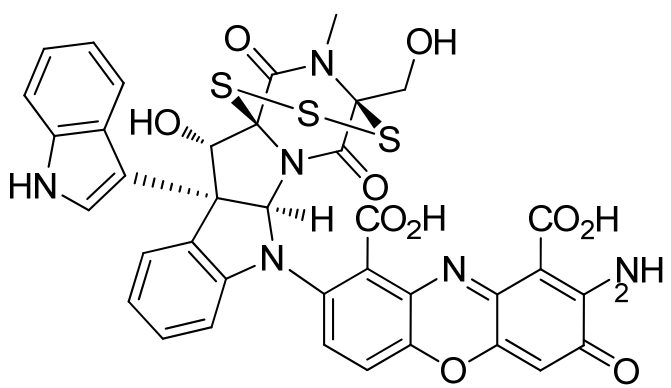

(586) plectosphaeroic acid C

Figure 103. Plectosphaeroic acids $\mathrm{A}-\mathrm{C}$.

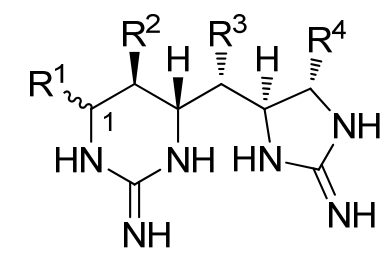

(587) araiosamine $A$ $\mathrm{R}^{1}=\mathrm{OH}, \mathrm{R}^{2,3,4}=6$-bromo[1H]indol-3-yl-, $1 R$ (588) araiosamine $\mathrm{B}$ $\mathrm{R}^{1}=\mathrm{OMe}, \mathrm{R}^{2,3,4}=6$-bromo[1H]indol-3-yl-, $1 S$

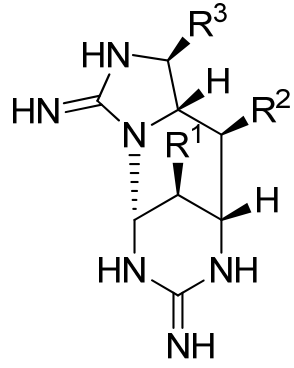

(589) araiosamine C

$\mathrm{R}^{1,2(3)}=6$-bromo[1H]indol-3-yl-

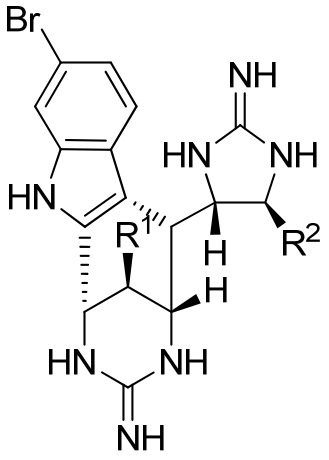

(590) araiosamine D

Figure 104. Araiosamines A-D.

Aspergilazine A (591), a dimeric DKP with and interesting connectivity, was isolated from the marine mangrove-derived fungus Aspergillus taichungensis ZHN-7-07 and had a weak antiviral activity towards influenza $\mathrm{A}\left(\mathrm{H}_{1} \mathrm{~N}_{1}\right)$ virus $(34.1 \%$ inhibition at $50 \mu \mathrm{g} / \mathrm{mL})$ (Figure 105) [253].<smiles>O=C1N[C@@H](Cc2cn(-c3ccc4c(C[C@H]5NC(=O)[C@H]6CCCN6C5=O)c[nH]c4c3)c3ccccc23)C(=O)N2CCC[C@@H]12</smiles>

(591) aspergilazine $A$

Figure 105. Aspergilazine A.

The polythiodioxopiperazines luteoalbusins A (592) and B (593) were obtained from Acrostalagmus luteoalbus SCSIO F457, isolated from deep-sea sediment, and showed potent cytotoxic activities against SF-268, MCF-7, NCI-H460 and HepG-2 cell lines (IC50 0.23-1.31 $\mu \mathrm{M}$ ) (Figure 106) [254]. 


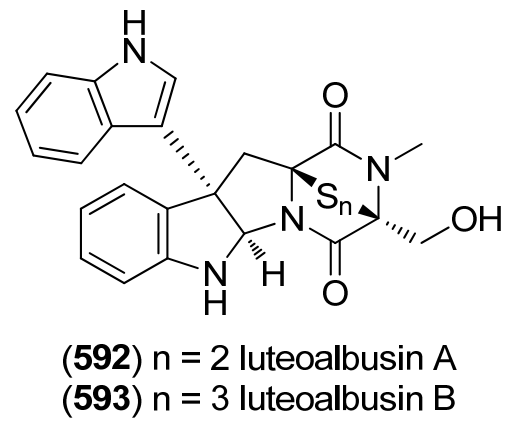

Figure 106. Luteoalbusins $\mathrm{A}$ and $\mathrm{B}$.

Racemosins A (594) and B (595), which may be biosynthetically derived from bisindole alkaloid caulerpin, were isolated from the green alga Caulerpa racemosa and showed neuro-protective activity against $A \beta_{25-35}$-induced SH-SY5Y cell damage (Figure 107) [255]. Racemosin C (596) [256] was isolated from the same organism and showed hPTP1B (human protein tyrosine phosphatase-1B) inhibitory activity ( $\mathrm{IC}_{50} 5.86 \mu \mathrm{M}$ ), as well as caulerchlorin (597) [257] which exhibited weak antifungal activity against Cryptococcus neoformans strain 32609 ( $\mathrm{MIC}_{80} 16 \mu \mathrm{g} / \mathrm{mL}$ ).

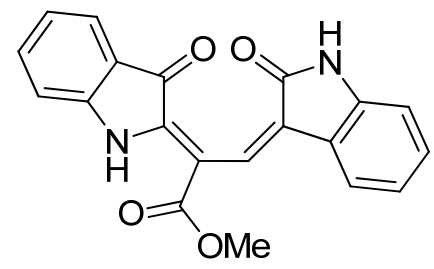

(594) racemosin A

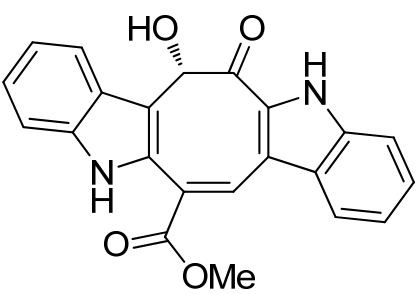

(596) racemosin C<smiles>COC(=O)c1cc2c3ccccc3[nH]c2c2c1[nH]c1ccccc12</smiles>

(595) racemosin B<smiles>COC(=O)/C1=C/c2c([nH]c3ccccc23)/C(Cl)=C\c2[nH]c3ccccc3c21</smiles>

(597) caulerchlorin

Figure 107. Racemosins and caulerchlorin.

Hyrtimomines A-K (598-608) were isolated from the Okinawan marine sponge Hyrtios sp. (Figure 108) [258-260]. Hyrtimomine A (598) displayed cytotoxic activity towards KB and L1210 cells [258]. Hyrtimomines D (601) and E (602) showed antimicrobial effects against Candida albicans, Cryptococcus neoformans, Staphylococcus aureus and Trichophyton mentagrophytes (MIC 4-16 $\mu \mathrm{g} / \mathrm{mL}$ ) [259]. Several antimicrobial activities were also reported for hyrtimomines A, B, F, G and I [260].

The marine actinomycete NPS12745, isolated from a marine sediment (San Diego, CA, USA), provided chlorinated bisindole pyrroles lynamicins A-E (609-613) (Figure 109). They exhibited a broad spectrum of antimicrobial activities, also against methicillin-resistant Staphylococcus aureus and vancomycin-resistant Enterococcus faecium [261]. The structurally reamarkable pyrrole-fused spirocyclic 
bisindole alkaloids spiroindimicins A-D (614-617) were isolated from the deep-sea-derived Streptomyces sp. SCSIO 03032. Spiroindimicins B-D (615-617) exhibited moderate cytotoxicity against the tumor cell lines CCRF-CEM, B16, HepG2 and H460 [262]. The same producer was also the source of indimicins A-E (618-622) as well as of lynamicins F (623) and G (624). None of these compounds exhibited antimicrobial activities, but indimicin B (619) showed cytotoxic effects against the MCF-7 breast cancer cell line [263]. The indimicins A-D are structurally similar to the staurosporin core but a planar arrangement is prevented by an angular methyl group. Spiroindimicins $\mathrm{C}$ (616), D (617) and lynamicin D (612) were proposed to be potent topoisomerase II, cathepsin K, cytochrome P450 3A4, aromatase P450, protein kinase and histone deacetylase inhibitors based on an in silico molecular docking approach [264].

Brocazines A-F (625-630), epidithiodiketopiperazines with a probable bigenetic relation to tryptophan, were isolated from the extract of the mangrove-derived Penicillium brocae MA-231 and exhibited cytotoxicity against several tumor cell lines (Figure 110) [265].<smiles></smiles>

(598) hyrtimomine A<smiles></smiles>

(601) $\mathrm{R}=\mathrm{H} \quad$ hyrtimomine $\mathrm{D}$ (602) $\mathrm{R}=\mathrm{CO}_{2} \mathrm{H}$ hyrtimomine $\mathrm{E}$<smiles></smiles><smiles></smiles>

(599) hyrtimomine B<smiles>O=C1NC(C(=O)c2c[nH]c3ccc(O)cc23)c2c(O)ccc3[nH]cc(c23)C1=O</smiles>

(603) hyrtimomine $\mathrm{F}$<smiles>O=C1CN=C(c2c[nH]c3ccc(O)cc23)c2c(O)ccc3[nH]cc1c23</smiles>

(600) hyrtimomine C<smiles>O=C(c1c[nH]c2ccc(O)cc12)C(O)[C@H](O)C(=O)c1c[nH]c2ccc(O)cc12</smiles>

(604) hyrtimomine G

(605) $\mathrm{R}^{1}=\mathrm{CO}_{2} \mathrm{H}, \mathrm{R}^{2}=\mathrm{H}_{2}, \mathrm{R}^{3}=\mathrm{S}(\mathrm{O})$ Me hyrtimomine $\mathrm{H}$

(606) $\mathrm{R}^{1}=\mathrm{CO}_{2} \mathrm{H}, \mathrm{R}^{2}=\mathrm{O}, \mathrm{R}^{3}=\mathrm{H} \quad$ hyrtimomine I

(607) $\mathrm{R}^{1}=\mathrm{CO}_{2} \mathrm{H}, \mathrm{R}^{2}=\mathrm{O}, \mathrm{R}^{3}=\mathrm{S}(\mathrm{O}) \mathrm{Me}$ hyrtimomine $\mathrm{J}$

(608) $\mathrm{R}^{1}=\mathrm{H}, \quad \mathrm{R}^{2}=\mathrm{O}, \mathrm{R}^{3}=\mathrm{S}(\mathrm{O}) \mathrm{Me}$ hyrtimomine $\mathrm{K}$

Figure 108. Hyrtimomines A-K. 
<smiles>[R]OC(=O)c1[nH]cc(-c2c[nH]c3cc([R])c(Cl)cc23)c1-c1c[nH]c2ccc(Cl)cc12</smiles>

(609) $\mathrm{R}=\mathrm{H}$ lynamicin $\mathrm{A}$ (610) $R=C l$ lynamicin $B$<smiles>COC(=O)c1[nH]c(C(=O)OC)c2c1C1=CNC3=CC=C(Cl)C=C1[C@@]32C=Nc1ccc(Cl)cc1</smiles>

(614) spiroindimicin A<smiles>[R]c1c2c(cn1[R1])[C@@]1(C)c3cc(Cl)ccc3N(C)[C@]1(C)c1[nH]c3ccc(Cl)cc3c1-2</smiles>

(618) $R^{1}=M e, R^{2}=H$ indimicin $A$ (619) $R^{1,2}=M e$ indimicin $B$ (620) $R^{1}=H, R^{2}=M e$ indimicin $C$ (621) $R^{1,2}=H$ indimicin $D$<smiles></smiles>

(611) lynamicin C<smiles>[R]OC(=O)c1[nH]c(C(=O)OC)c(-c2c[nH]c3ccc(Cl)cc23)c1-c1c[nH]c2ccc([R])cc12</smiles>

(612) $R=$ Cl lynamicin $D$ (613) $R=H$ lynamicin $E$<smiles>[R]c1[nH]c(C(=O)OC)c2c1-c1c([nH]c3ccc(Cl)cc13)[C@@]21CN([R1])c2ccc(C)cc21</smiles>

(615) $R^{1}=$ Me, $R^{2}=H$ spiroindimicin $B$ (616) $R^{1,2}=H$ spiroindimicin $C$ (617) $\mathrm{R}^{1}=\mathrm{Me}, \mathrm{R}^{2}=\mathrm{CO}_{2} \mathrm{Me}$ spiroindimicin $\mathrm{D}$<smiles>Cn1cc(-c2c[nH]c3ccc(Cl)cc23)c([C@@]2(C)CNc3ccc(Cl)cc32)c1</smiles>

(622) indimicin E<smiles>[R]c1c(-c2c[nH]c3ccc(Cl)cc23)c(-c2c[nH]c3ccc(Cl)cc23)c(C(=O)OC)n1C</smiles>

(623) $\mathrm{R}=\mathrm{H}$ lynamicin $\mathrm{F}$

(624) $\mathrm{R}=\mathrm{CO}_{2} \mathrm{Me}$ lynamicin $\mathrm{G}$

Figure 109. Lynamicins $A-G$, spiroindimicins $A-D$ and indimicins $A-E$.

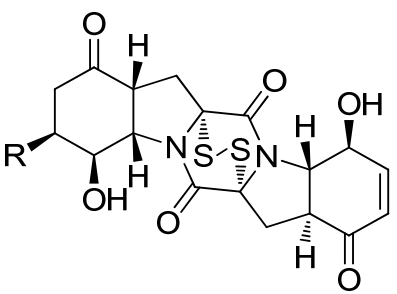

(625) $\mathrm{R}=$ OMe brocazine $A$ (626) $\mathrm{R}=\mathrm{H} \quad$ brocazine $\mathrm{B}$

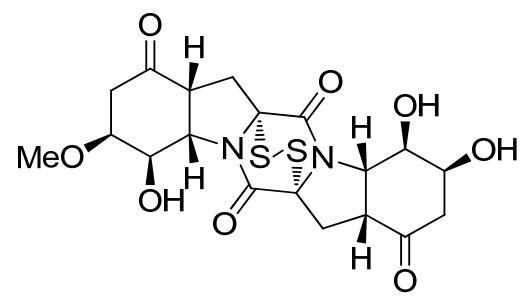

(628) brocazine $\mathrm{D}$

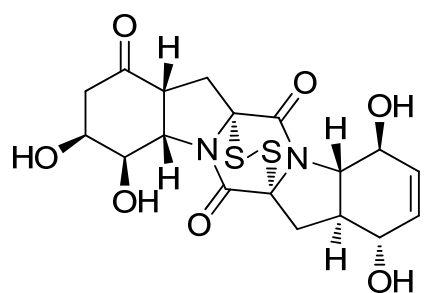

(627) brocazine $\mathrm{C}$

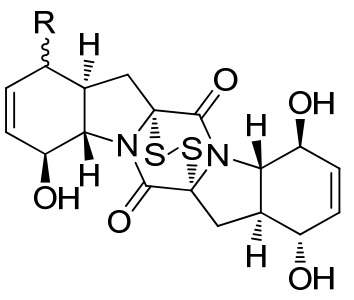

(629) $\mathrm{R}=\alpha-\mathrm{OH}$ brocazine $\mathrm{E}$ (630) $\mathrm{R}==\mathrm{O}$ brocazine $\mathrm{F}$

Figure 110. Brocazines A-F. 


\subsection{Anellated Indoles}

In the anellated indole alkaloids, a single indole core is fused to other (hetero)cyclic ring systems that are not prenyl derived. For example, the discorhabdins L (631) and I (632) were isolated from the marine sponge Latrunculia brevis and exhibited potent cytotoxic activity towards 14 tumor cell lines (e.g., HT-29 colon cell line: GI50 0.12 and $0.35 \mu \mathrm{M}$, respectively) (Figure 111) [266]. The discorhabdin derivatives 3-dihydro-7,8-dehydrodiscorhabdin C (633), 14-bromo-3-dihydro-7,8-dehydrodiscorhabdin C (634), discorhabdin V (635), 14-bromo-1-hydroxydiscorhabdin V (636), tsitsikammamine A $N-18$ oxime (637), tsitsikammamine B N-18 oxime (638), 1-methoxydiscorhabdin D (639), and 1-aminodiscorhabdin D (640) were isolated from extracts of four South African latrunculid sponges, Tsitsikamma pedunculata, T. favus, Latrunculia bellae, and Strongylodesma algoaensis. (633-635), (639) and (640) showed cytotoxicity against human colon tumor HCT-116 cells. [267] Discorhabdin W (641), the first dimeric congener, was obtained from a New Zealand sponge Latrunculia sp. and showed strong cytotoxic effects towards P388 leukemia cells [268]. (+)-Dihydrodiscorhabdin A (642), (+)-debromodiscorhabdin A (643), (+)-dihydrodiscorhabdin L (644) and (+)-discorhabdin X (645) were obtained from southern Australian marine sponges of the genera Higginsia and Spongosorites [269]. Dihydrodiscorhabdin B (646) and discorhabdin Y (647) were isolated from a deep-water Alaskan sponge (Latrunculia sp.) [270]. (-)-3-Dihydrodiscorhabdin D (648) and (-)-discorhabdin Z (649) were isolated from the Korean marine sponge Sceptrella sp. [271]. Tsitsikammamine C (650) was isolated from the Australian marine sponge Zyzzya sp. and exhibited strong cytotoxic and antiparasitic activity [272].

$N$-1- $\beta$-D-Ribofuranosyldamirone $\mathrm{C}$ (651) and $N$-1- $\beta$-D-ribofuranosylmakaluvamine I (652) were obtained from the South African latrunculid sponge Strongylodesma aliwaliensis and showed activity against esophageal cancer cell lines WHCO1, WHCO6, and KYSE30 (IC50 1.6-85.5 $\mu \mathrm{M})$ (Figure 112) [273,274].

Zyzzyanones A (653) [275] and B-D (654-656) [276], bearing a pyrrolo[3,2-f]indole-4,8(1H,7H)dione skeleton, were isolated from the Australian marine sponge Zyzzya fuliginosa (Figure 113). Zyzzyanones A-D (653-656) exhibit moderate cytotoxic activity against mouse Ehrlich carcinoma cells, as well as UV-protective activity [275-277].

Ophiuroidine (657) has an indolo[2,1-b]quinazoline-6,12-dione skeleton and was obtained from the Caribbean ophiuroid Ophiocoma riisei (Figure 114) [278].

Fumiquinazoline $\mathrm{J}$ (658) was isolated from the marine-derived fungal strain Aspergillus fumigatus H1-04 and exhibited cytotoxic activities against the cell lines tsFT210, P388, HL-60, A549 and BEL-7402 (Figure 115) [279]. Fumiquinazolines K-P (659-664), together with tryptoquivaline K (668, Figure 116), were isolated from the Mediterranean sponge-derived fungi Aspergillus sp. [280] Compounds 665 and 666 were simultaneously isolated from the soft coral-derived fungus $A$. fumigatus KMM 4631 and a gorgonian-derived fungus (Scopulariopsis sp.), respectively, and were initially doubly designated as fumiquinazolines $\mathrm{K}$ and $\mathrm{L}$ [281,282]. These names have been revised to fumiquinazolines Q (665) and R (666). Fumiquinazoline S (667) was isolated from marine-derived Aspergillus sp., together with isochaetominines A-C (669-671) and 14-epi-isochaetominine C (672) (Figure 116). Fumiquinazolines F, L (660), S (667) and isochaetominines (669-672) showed weak inhibition against $\mathrm{Na}^{+} / \mathrm{K}^{+}$-ATPase (IC50 17-78 $\left.\mu \mathrm{M}\right)$ [283]. 


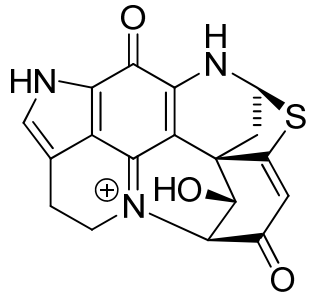

(631) discorhabdin L

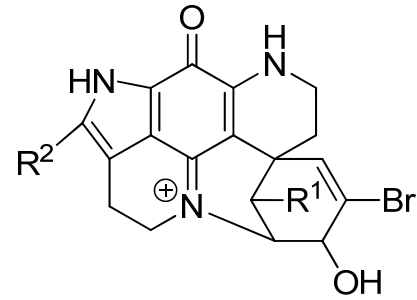

(635) $\mathrm{R}^{1,2}=\mathrm{H}$

(636) $\mathrm{R}^{1}=\mathrm{OH}, \mathrm{R}^{2}=\mathrm{Br}$

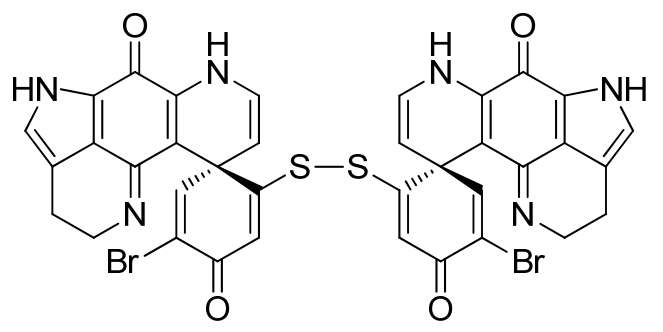

(641) discorhabdin W<smiles></smiles>

(643) (+)-debromodiscorhabdin A<smiles>O=C1CC2=CC(O)C(Br)C=C2C2=C1c1c3c[nH]c1C(NCC3)S2</smiles>

(646) dihydrodiscorhabdin B

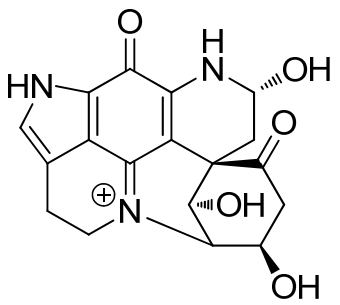

(649) discorhabdin Z

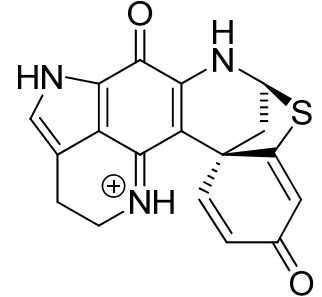

(632) discorhabdin I<smiles>[R]n1cc2c3c1C(=O)c1[nH]cc(-c4ccc(C)cc4)c1C3=[N+](O)CC2</smiles>

(637) $\mathrm{R}=\mathrm{H} \quad \mathrm{OH}$

(638) $\mathrm{R}=\mathrm{Me}$<smiles></smiles>

(633) $\mathrm{R}=\mathrm{H}$

(634) $\mathrm{R}=\mathrm{Br}$

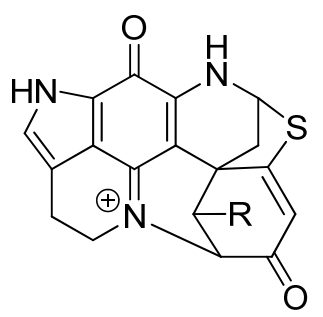

(639) $\mathrm{R}=\mathrm{OMe}$

(640) $\mathrm{R}=\mathrm{NH}_{2}$<smiles>O=C1C2=C(C3=NCCc4c[nH]c1c43)C13C=C(Br)[C@@H](O)CC1SC2N3</smiles>

(642) (+)-dihydrodiscorhabdin A

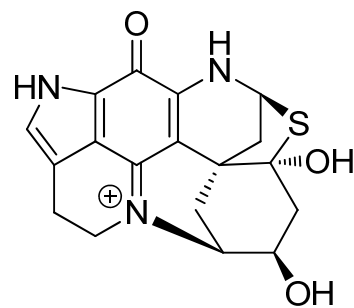

(645) discorhabdin X

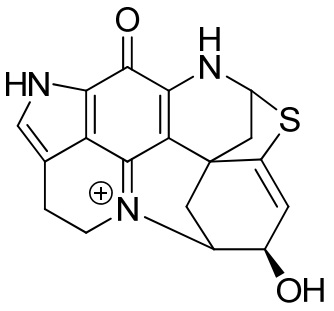

(648) (-)-3-dihydrodiscorhabdin D

Figure 111. Discorhabdin and tsitsikammamine derivatives. 
<smiles></smiles>

(651) N-1- $\beta$-D-ribofuranosyldamirone C<smiles></smiles>

(652) N-1- $\beta$-D-ribofuranosylmakaluvamine I

Figure 112. $N$-1- $\beta$-D-Ribofuranosyldamirone $\mathrm{C}$ and $N$-1- $\beta$-D-ribofuranosylmakaluvamine $\mathrm{I}$.<smiles></smiles>

(653) $R=$ Me zyzzyanone A (654) $\mathrm{R}=\mathrm{H}$ zyzzyanone $B$<smiles></smiles>

(655) $\mathrm{R}=$ Me zyzzyanone $\mathrm{C}$ (656) $\mathrm{R}=\mathrm{H}$ zyzzyanone $\mathrm{D}$

Figure 113. Zyzzyanones A-D.<smiles></smiles>

(657) ophiuroidine

Figure 114. Ophiuroidine. 
<smiles>C[C@H]1N[C@@]2(C[C@H](C3C=Nc4ccccc4C3=O)C(=O)O2)[C@]2(C)C(=O)N1c1ccccc12</smiles>

(658) fumiquinazoline $\mathrm{J}$<smiles>Cc1c(O)c(C(=O)O)c(O)c2c1[C@H](C)[C@@H](C)O[C@H]2C=C1NC(=O)[C@@H](C[C@@]2(O)c3ccccc3N3C(=O)C4(CC4)N[C@@H]32)n2c1nc1ccccc1c2=O</smiles>

(661) fumiquinazoline $M$<smiles>CC12NC(=O)C3(Cc4[nH]c5ccccc5c4CC13)n1c2nc2ccccc2c1=O</smiles>

(665) fumiquinazoline $Q$<smiles>CN1[C@H]2C[C@@]3(O)C(=Nc4ccccc4C(=O)C2=O)[C@@H]1CN1N(C(=O)C12CC2)c1ccccc13</smiles>

(659) fumiquinazoline $\mathrm{K}$<smiles>[R][C@]1(C)c2nc3ccccc3c(=O)n2[C@H](C[C@]2(O)c3ccccc3N3C(=O)C4(CC4)N(C(C)=O)[C@@H]32)C(=O)N1C</smiles>

(662) $\mathrm{R}=\mathrm{OH}$ fumiquinazoline $\mathrm{N}$ (663) $\mathrm{R}=\mathrm{OMe}$ fumiquinazoline $\mathrm{O}$

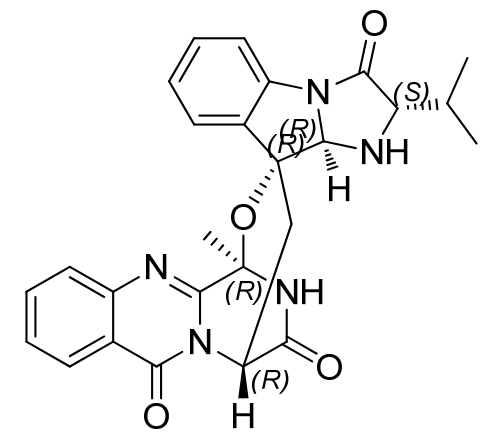

(666) fumiquinazoline $\mathrm{R}$<smiles>C[C@]1(O)NC(=O)[C@@H](C[C@]2(O)c3ccccc3N3C(=O)C4(CC4)N[C@@H]32)n2c1nc1ccccc1c2=O</smiles>

(660) fumiquinazoline $L$<smiles>C=C1c2nc3ccccc3c(=O)n2C(C[C@]2(O)c3ccccc3N3C(=O)C4(CC4)N(C(C)=O)[C@H]32)C(=O)N1C</smiles>

(664) fumiquinazoline $P$

Figure 115. Fumiquinazolines $\mathrm{K}-\mathrm{S}$.<smiles>CC(=O)N1[C@@H]2N(C(=O)C13CC3)c1ccccc1[C@@]21C[C@@H](n2cnc3ccccc3c2=O)C(=O)O1</smiles>

(668) tryptoquivaline $\mathrm{K}$<smiles>[R]C1C(=O)N2c3ccccc3[C@@]3(O)C[C@H](n4cnc5ccccc5c4=O)C(=O)N1[C@@H]23</smiles>

(669) $\mathrm{R}=\mathrm{Me}(14 R)$ isochaetominines $\mathrm{A}$ (670) $\mathrm{R}=\mathrm{Et}(14 R)$ isochaetominines $\mathrm{B}$ (671) $\mathrm{R}={ }^{i} \operatorname{Pr}(14 R)$ isochaetominines $\mathrm{C}$ (672) $\mathrm{R}={ }^{i} \operatorname{Pr}(14 S)$ 14-epi-isochaetominine $\mathrm{C}$

Figure 116. Tryptoquivaline $\mathrm{K}$, isochaetominines $\mathrm{A}-\mathrm{C}$ and 14-epi-isochaetominine $\mathrm{C}$. 
Cottoquinazolines A-D (673-676) were obtained from the marine-derived fungus Aspergillus versicolor strains MST-MF495 and LCJ-5-4 (Figure 117). Cottoquinazoline D (676) has been reported to show antifungal activity against Candida albicans (MIC 22.6 $\mu \mathrm{M}$ ) [284,285].<smiles>C[C@H]1C(=O)N2c3ccccc3[C@@]3(O)CC4C(=O)NC1N4C23O</smiles>

(673) cottoquinazoline A

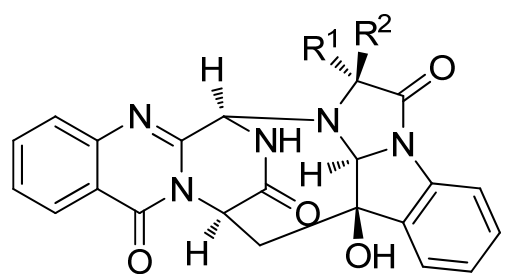

(674) $R^{1}=M e, R^{2}=H$ cottoquinazoline $B$

(675) $\mathrm{R}^{1}=\mathrm{H}, \mathrm{R}^{2}=$ cottoquinazoline $\mathrm{C}$

(676) $\mathrm{R}^{1} \mathrm{R}^{2}=\mathrm{CH}_{2} \mathrm{CH}_{2}$ cottoquinazoline $\mathrm{D}$

Figure 117. Cottoquinazolines A-D.

Fumiquinazolines fumigatosides B-D (677-679) were obtained from the fungus Aspergillus fumigatus derived from the giant Japanese jellyfish Nemopilema nomurai (Figure 118). They are biosynthetically derived from anthranilic acid, tryptophan, L-alanine and D-glucose. Neither antibacterial nor cytotoxic effects were observed [286].

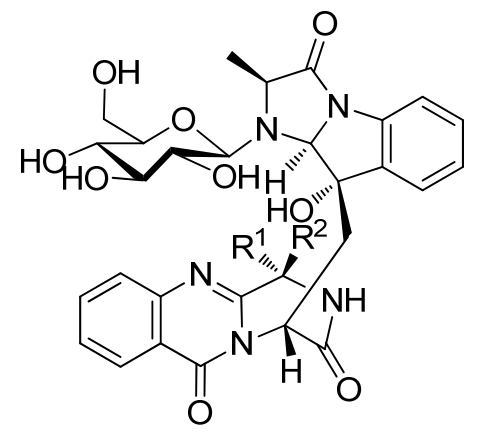

(677) $\mathrm{R}^{1}=\mathrm{Me}, \mathrm{R}^{2}=\mathrm{OH}$ fumigatoside $\mathrm{B}$

(678) $R^{1}=H, R^{2}=M e$ fumigatoside $C$

(679) $R^{1}=M e, R^{2}=H$ fumigatoside $D$

Figure 118. Fumigatosides B-D.

Antipathine A (680) was isolated from the South China Sea black coral Antipathes dichotoma and exhibited cytotoxic effects towards the human carcinoma cell lines SGC-7901 and Hep-G2 (Figure 119) [287]. Azonazine (681), which contains a unique hexacyclic bridged diketopiperazine structure, was obtained from the Hawaiian marine sediment-derived fungus Aspergillus insulicola and exhibited anti-inflammatory activity via inhibition of NF- $\kappa \mathrm{B}$ (nuclear factor kappa-light-chain-enhancer of activated B cells) luciferase and nitrite production [288]. 
<smiles>CN1C(=O)c2cc(cc3c2[nH]c2ccccc23)N(C)C1=O</smiles>

(680) antipathine A<smiles>CC(=O)N1C(=O)[C@]2(CC3NC(=O)[C@H]4Cc5cccc(c5N4)O[C@H]32)c2ccccc21</smiles>

(681) azonazine

Figure 119. Antipathine A and azonazine.

Protubonines A (682) and B (683) were isolated from the marine-derived fungus Aspergillus sp. SF-5044 and showed no cytotoxic effects against the tumor cell lines HL-60, MDA-MB-231, Hep3B, $3 \mathrm{Y} 1$ and $\mathrm{K} 562\left(\mathrm{IC}_{50}>250 \mu \mathrm{M}\right)$ (Figure 120) [289].<smiles>CC(=O)N1c2ccccc2[C@]2(O)C[C@H]3C(=O)N[C@@H](CC(C)C)C(=O)N3[C@H]12</smiles>

(682) protubonine A<smiles>CC(=O)O[C@]12C[C@H]3C(=O)N[C@H](CC(C)C)C(=O)N3[C@H]1N(C(C)=O)c1ccccc12</smiles>

(683) protubonine B

Figure 120. Protubonines A and B.

Aniquinazolines A-D (684-687) were obtained from the marine-derived Aspergillus nidulans MA-143 and displayed potent toxicity against brine shrimp (Artemia salina, LC50 1.27, 2.11, 4.95 and $3.42 \mu \mathrm{M}$, respectively), but did not show antibacterial and cytotoxic effects (Figure 121) [290].

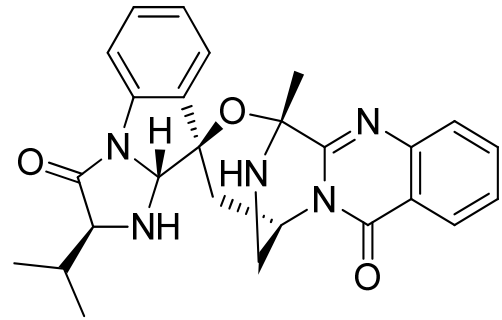

(684) aniquinazoline $A$

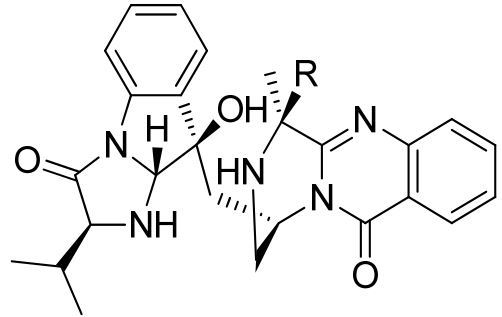

(685) $\mathrm{R}=\mathrm{H}$ aniquinazoline $\mathrm{B}$ (686) $\mathrm{R}=\mathrm{OH}$ aniquinazoline $\mathrm{C}$<smiles></smiles>

(687) aniquinazoline D

Figure 121. Aniquinazolines A-D.

7-Bromo-1-(6-bromo-1H-indol-3-yl)-9H-carbazole $\mathbf{( 6 8 8 )}$ and 3,11-dibromo-13H-indolo[3,2$k$ ]phenanthridine (689) were isolated from the marine sponge Penares sp. (South China Sea) (Figure 122). 7-Bromo-1-(6-bromo-1H-indol-3-yl)-9H-carbazole (688) exhibited cytotoxicity towards HL-60 and HeLa human tumor cells (IC 5016.1 and $33.2 \mu \mathrm{M}$, respectively) [291]. 
<smiles>Brc1ccc2c(-c3cccc4c3[nH]c3cc(Br)ccc34)c[nH]c2c1</smiles><smiles>Brc1ccc2c(c1)Nc1c(ccc3cc(Br)ccc13)C=N2</smiles>

Figure 122. 7-Bromo-1-(6-bromo- $1 H$-indol-3-yl)-9H-carbazole and 3,11-dibromo-13Hindolo[3,2-k]phenanthridine.

Tryptoquivalines $\mathrm{P}-\mathrm{S}(\mathbf{6 9 0}-\mathbf{6 9 3})$ have been isolated from a marine-derived fungus Neosartorya sp. HN-M-3 (Figure 123) [292,293].<smiles>COC(=O)[C@@H](C[C@@]1(O)c2ccccc2N2C(=O)C(C)(C)NC21C)n1cnc2ccccc2c1=O</smiles>

(690) tryptoquivaline $\mathrm{P}$<smiles>C[C@H]1C(=O)N2O[C@@H]3OC(=O)[C@@](C[C@@H]3n3cnc4ccccc4c3=O)(c3ccccc32)N1C=O</smiles>

(691) tryptoquivaline $Q$<smiles>COC1=C(n2cnc3ccccc3c2=O)C[C@@]2(O)c3ccccc3N2C(=O)C(C)(C)N1O</smiles>

(692) tryptoquivaline $R$<smiles>[CH][C@@]12N(C(=O)C(C)(C)N1O)c1ccccc1C1(O)CC(NC(=O)c3ccccc3NC(=O)C(O)C(C)C)=C(OC)C(=O)[C@]12O</smiles>

(693) tryptoquivaline S

Figure 123. Tryptoquivalines $\mathrm{P}-\mathrm{S}$.

Analysis of the Australian spider sponge Trikentrion flabelliforme led to the isolation of trikentramides A-D (694-697) (Figure 124). Until now, no biological evaluation of these compounds has been reported [294].<smiles>CCc1cc2c(c3c1C(=O)C(=O)N3)[C@H](C)C[C@@H]2C</smiles>

(694) trikentramide A<smiles>CCc1cc2c(c3c1C(=O)C(=O)N3)[C@H](C)C[C@@H]2C</smiles>

(695) trikentramide B<smiles>Cc1cc2c(c3c1C(C)C[C@@H]3C)NC(=O)C2=O</smiles>

(696) trikentramide C<smiles>Cc1cc2c(c3c1C(C)C[C@@H]3C)NC(=O)C2</smiles>

(697) trikentramide D

Figure 124. Trikentramides A-D.

Nigrospin A (698) was recently isolated from the marine-derived fungus Nigrospora oryzae SCSGAF 0111, the terrestrial forms of which are known plant pathogens (Figure 125) [295]. 
<smiles>CC1(C)Oc2ccc3c(C(=O)O)c[nH]c3c2[C@H](O)[C@@H]1O</smiles>

(698) nigrospin A

Figure 125. Nigrospin A.

Hyrtioreticulins A-F were isolated from Hyrtios reticulatus. Hyrtioreticulins A, B, E and F (779-782) possess a $\beta$-carboline framework (see Figure 143), hyrtioreticulins C (699) and D (700) belong to the group of azepino-indole-type alkaloids (Figure 126) [296,297].<smiles>C[C@H]1N[C@@H](C(=O)O)Cc2c[nH]c3ccc(O)c1c23</smiles>

(699) $6 R$ hyrtioreticulin C

(700) $6 S$ hyrtioreticulin D

Figure 126. Hyrtioreticulins A-F.

Aspergillus versicolor dl-29, isolated from the marine green alga Codium fragile, was the source of aspeverin (701), which inhibits the growth of the phytoplankton microalga Heterosigma akashiwo as well as brine shrimp Artemia salina and the bacterial species Vibrio ichthyoenteri, Proteus mirabilis, Enterobacter cloacae and Bacillus cereus (Figure 127) [298].

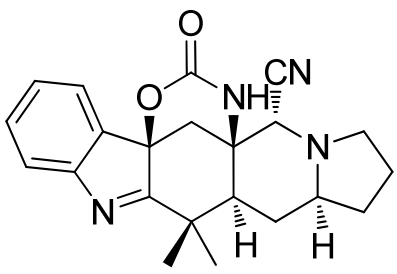

(701) aspeverin

Figure 127. Aspeverin.

Cyanogramide (702), a spirocyclic pyrrolo[1,2-c]imidazole, was obtained from Actinoalloteichus cyanogriseus WH1-2216-6 and displayed weak cytotoxic effects towards K562, MCF-7, KB, and the MDR cell lines K562/A02, MCF-7/Adr and KB/VCR (IC50 12.9, 18.5, 16.8, 10.2, 36.0 and $25.6 \mu \mathrm{M}$, respectively) and could reverse the multidrug resistance of K562/A02, MCF-7/Adr, and KB/VCR cells (Figure 128) [299]. $N$-Formyllapatin A (703) was identified from Penicillium adametzioides and did not show any appreciable antibacterial activity [300]. 


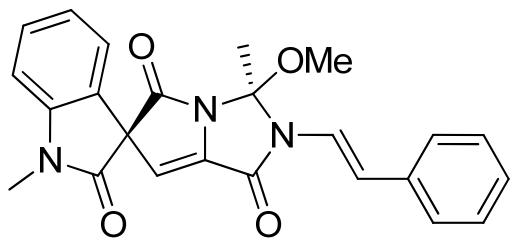

(702) cyanogramide

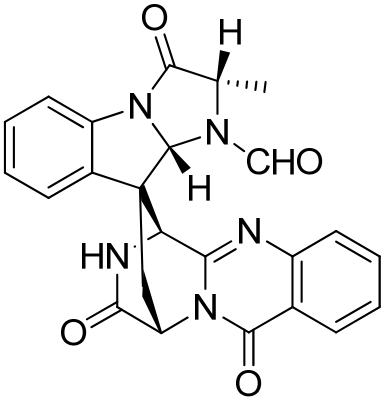

(703) N-formyllapatin A

Figure 128. Cyanogramide and $N$-formyllapatin A.

Spiroindolinone alkaloids cycloexpansamines A (704) and B (705) were obtained from a marine isolate of Penicillium sp. (SF-5292) (Figure 129) [301].<smiles>C[C@@H]1[C@@H](C)C2(CC13CN1C(=O)N4CCCC41C3)C(=O)N1c3c2ccc(O)c3C(=O)CC1(C)C</smiles>

(704) cycloexpansamine A<smiles>C[C@@H]1[C@@H]2C[C@@H]3CCCN3C[C@@]2([N+](=O)[O-])CC12C(=O)N1c3c2ccc(O)c3C(=O)CC1(C)C</smiles>

(705) cycloexpansamine B

Figure 129. Cycloexpansamines A and B.

The Epidithiodiketopiperazine deoxyapoaranotin (706), together with acetylaranotin and acetylapoaranotin, was separated from Aspergillus sp. KMD 901, which was obtained from a marine sediment from the East Sea of Korea (Figure 130). Deoxyapoaranotin (706) was found to have cytotoxic activity towards HCT-116 colon cancer cell line via apoptosis-inducing effects [302].

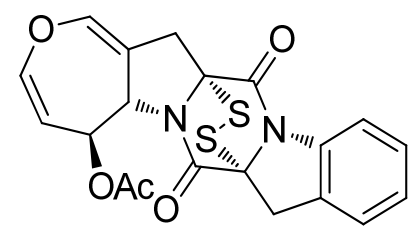

(706) deoxyapoaranotin

Figure 130. Deoxyapoaranotin.

The gliotoxin derivative dehydroxybisdethiobis(methylthio)gliotoxin (707) was isolated from the marine-derived fungus Pseudallescheria sp. (Figure 131) [303]. Bis(dethio)-10a-methylthio-3a-deoxy3,3a-didehydrogliotoxin (708) and 6-deoxy-5a,6-didehydrogliotoxin (709), were obtained from the Japanese deep sea-derived fungus Penicillium sp. strain JMF034 and 6-deoxy-5a,6-didehydrogliotoxin (709) showed cytotoxic effects against P388 murine leukemia cells ( $\mathrm{IC}_{50} 3.4$ and $\left.0.058 \mu \mathrm{M}\right)$ [304]. 
<smiles>CN1C(=O)[C@]2(S(C)(=O)=O)CC3=CC=C[C@H](O)[C@H]3N2C(=O)C1(C)C</smiles>

(707)<smiles>C=C1C(=O)N2[C@@H](CC3=CC=C[C@H](O)[C@@H]32)C(=O)N1C</smiles>

(708)<smiles>CN1C(=O)C23Cc4ccccc4N2C(=O)C1(CO)SS3</smiles>

(709)

Figure 131. Gliotoxin derivatives.

Phomazines A-C (710-712) were isolated from the mangrove-derived endophytic fungus, Phoma sp. OUCMDZ-1847 (Figure 132). Phomazine B (711) exhibited moderate cytotoxic activities towards cancer cell lines HL-60, HCT-116, K562, MGC-803 and A549 (IC50 between 8.5 and >10 $\mu$ M) [305].<smiles>C[C@]12CC3=CC=C[C@H](O)[C@H]3N1C(=O)/C(=C/c1ccccc1)NC2=O</smiles>

(710) phomazine A<smiles>CS[C@@]1(Cc2ccccc2)NC(=O)[C@]2(SC)CC3=CC=C[C@H](O)[C@H]3N2C1=O</smiles>

(711) phomazine B

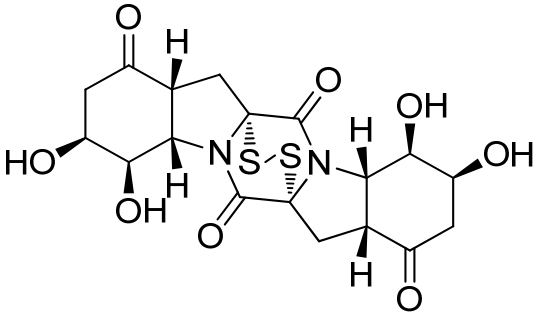

(712) phomazine C

Figure 132. Phomazines A-C.

$\beta$-Carbolines

$\beta$-Carbolines are a large group of indole alkaloids and widespread in nature. Their producers include plants, insects, marine organisms, etc. They provide a large spectrum of pharmacological activities [306].

Thorectandramine (713) was obtained from the Palauan sponge Thorectandra sp. and exhibited weak cytotoxic activity towards MCF-7, OVCAR-3 and A549 cell lines (Figure 133) [307].<smiles></smiles>

(713) thorectandramine

Figure 133. Thorectandramine.

3-Bromofascaplysin (714), 14-bromoreticulatine (715) and 14-bromoreticulatate (716) were isolated from collections of the sponge Fascaplysinopsis reticulata and the tunicate Didemnum sp. (Figure 134) [308]. 3-Bromofascaplysin (714) was found to exhibit anticancer activity towards the cell lines HL-60, THP-1, HeLa, MDA-MB-231, DLD-1, SNU-C4 and SK-MEL-28, which was identified 
to be caspase-3, -8 and -9-mediated [309]. 717 and 1-deoxysecofascaplysin A (718) were obtained from the marine sponge Thorectandra sp. and showed cytotoxic activities [310]. 10-Bromofascaplysin (719), 3,10-dibromofascaplysin (720), homofascaplysate A (721), homofascaplysin B-1 (722), 3-bromohomofascaplysins B (723), B-1 (724) and C (725), 7,14-dibromoreticulatine (726), reticulatol (727), 14-bromoreticulatol (728) and 3-bromosecofascaplysins A (729) and B (730) were isolated from four collections of sponge Fascaplysinosis reticulata and two Didemnum sp. tunicates [311].<smiles></smiles>

(714) $R^{1}=\mathrm{Br}, \mathrm{R}^{2}=\mathrm{H}$ 3-bromofascaplysin (719) $R^{1}=H, R^{2}=B r$ 10-bromofascaplysin (720) $\mathrm{R}^{1,2}=\mathrm{Br} 3,10$-dibromofascaplysin<smiles></smiles>

(717)<smiles>[R]c1ccc2c([R])c3c4[nH]c5ccccc5c4ccn3c2c1</smiles><smiles>[R1]c1ccc([R])c(-[n+]2ccc3c(c2)[nH]c2cc([R1])ccc23)c1</smiles>

(715) $\mathrm{R}^{1}=\mathrm{H}, \mathrm{R}^{2}=\mathrm{CO}_{2} \mathrm{CH}_{3}, \mathrm{R}^{3}=\mathrm{Br}$ 14-bromoreticulatine

(716) $\mathrm{R}^{1}=\mathrm{H}, \mathrm{R}^{2}=\mathrm{CO}_{2} \mathrm{H}, \mathrm{R}^{3}=\mathrm{Br}$ 14-bromoreticulatate (726) $\mathrm{R}^{1}=\mathrm{Br}, \mathrm{R}^{2}=\mathrm{CO}_{2} \mathrm{CH}_{3}, \mathrm{R}^{3}=\mathrm{Br}$ 7,14-dibromoreticulatine (727) $\mathrm{R}^{1}=\mathrm{H}, \mathrm{R}^{2}=\mathrm{OH}, \mathrm{R}^{3}=\mathrm{H}$ reticulatol (728) $R^{1}=H, R^{2}=O H, R^{3}=\mathrm{Br}$ 14-bromoreticulatol<smiles></smiles>

(718) 1-deoxysecofascaplysin A<smiles></smiles>

(721) homofascaplysate A<smiles>[R]OC(=O)c1ccc(Br)cc1-n1ccc2c([nH]c3ccccc32)c1=O</smiles>

(722) $\mathrm{R}^{1}=\mathrm{H}, \mathrm{R}^{2}=\mathrm{C}(\mathrm{O}) \mathrm{CO}_{2} \mathrm{CH}_{2} \mathrm{CH}_{3}$ homofascaplysin $\mathrm{B}-1$

(723) $\mathrm{R}^{1}=\mathrm{Br}, \mathrm{R}^{2}=\mathrm{C}(\mathrm{O}) \mathrm{CO}_{2} \mathrm{CH}_{3}$ 3-bromohomofascaplysin $\mathrm{B}$

(724) $\mathrm{R}^{1}=\mathrm{Br}, \mathrm{R}^{2}=\mathrm{C}(\mathrm{O}) \mathrm{CO}_{2} \mathrm{CH}_{2} \mathrm{CH}_{3}$ 3-bromohomofascaplysin $\mathrm{B}-1 \quad$ (729) $\mathrm{R}=$ Me 3-bromosecofascaplysin A

(725) $\mathrm{R}^{1}=\mathrm{Br}, \mathrm{R}^{2}=\mathrm{C}(\mathrm{O}) \mathrm{H}$ 3-bromohomofascaplysin $\mathrm{C}$

(730) $R=H$ 3-bromosecofascaplysin $B$

Figure 134. Fascaplysin and reticulatine derivatives.

Tiruchanduramine (731) was isolated from the Indian ascidian Synoicum macroglossum and was reported to be a promising $\alpha$-glucosidase inhibitor ( $\mathrm{IC}_{50} 78.2 \mu \mathrm{g} / \mathrm{mL}$ ) (Figure 135) [312].<smiles>N=C1NCC(CNC(=O)c2cc3c(cn2)[nH]c2ccccc23)N1</smiles>

(731) tiruchanduramine

Figure 135. Tiruchanduramine. 
Manadomanzamines A (732) and B (733) were obtained from the Indo-Pacific sponge Acanthostrongylophora sp. and were found to exhibit strong activity against Mycobacterium tuberculosis and HIV-1 (Figure 136). Additionally, they show antifungal and cytotoxic, but only weak antimalarial, activity [313]. Furthermore, manzamine-type alkaloids 12,28-oxamanzamine A (734), 12,28-oxa-8hydroxymanzamine A (735), 12,34-oxamanzamine E (736), 8-hydroxymanzamine J (737), 6-hydroxymanzamine E (738), 12,28-oxamanzamine E (739), 12,34-oxa-6-hydroxymanzamine E (740) and 8-hydroxymanzamine B (741) have been isolated from Acanthostrongylophora sp. [314-316].

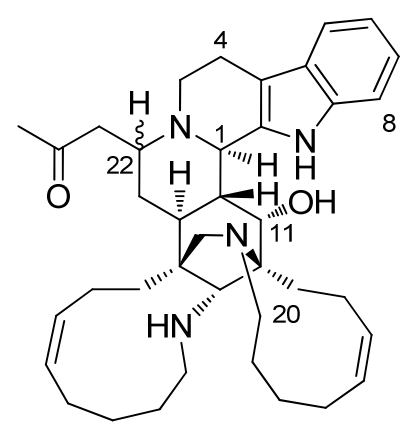

(732) 22- $\beta-\mathrm{H}$ manadomanzamine A (733) 22- $\alpha-\mathrm{H}$ manadomanzamine $B$

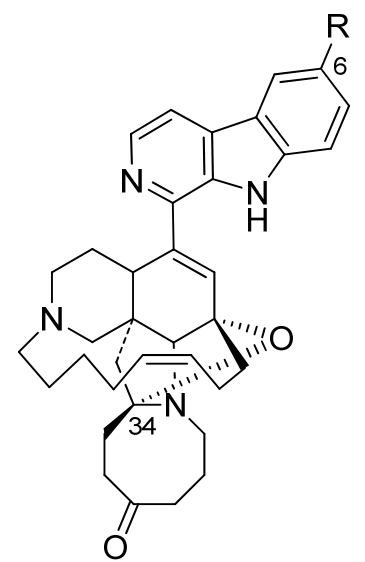

(736) $\mathrm{R}=\mathrm{H}$ 12,34-oxamanzamine $\mathrm{E}$ (740) $\mathrm{R}=\mathrm{OH}$ 12,34-oxa-6-hydroxymanzamine $\mathrm{E}$

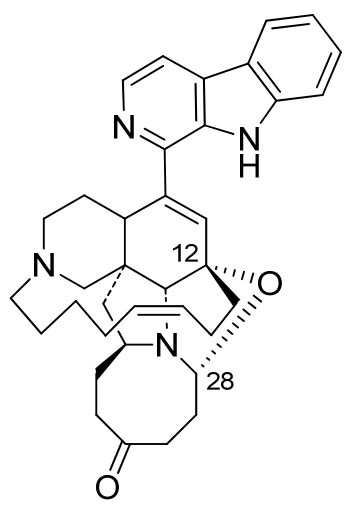

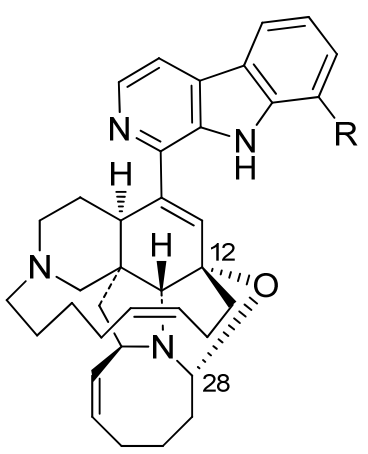

(734) $\mathrm{R}=\mathrm{H}$ 12,28-oxamanzamine $\mathrm{A}$

(735) $\mathrm{R}=\mathrm{OH} 12,28-\mathrm{oxa}-8$-hydroxymanzamine A

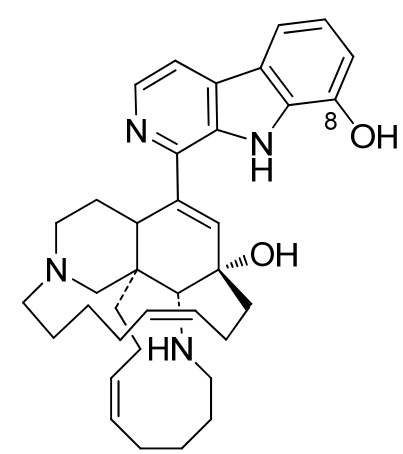

(737) 8-hydroxymanzamine J

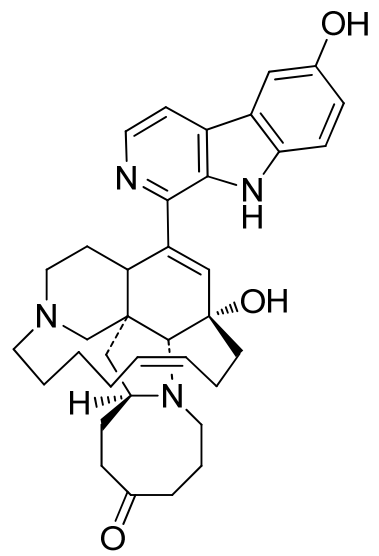

(738) 6-hydroxymanzamine E

(739) 12,28-oxamanzamine E

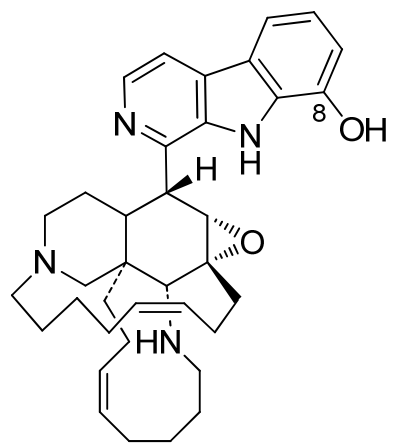

Figure 136. Manadomanzamines $\mathrm{A}$ and $\mathrm{B}$ and manzamine derivatives. 
Zamamidines A-C (742-744), 3,4-dihydro-6-hydroxy-10,11-epoxymanzamine A (745) and 3,4-dihydromanzamine $\mathrm{J} N$-oxide (746) were obtained from the Okinawan marine sponge Amphimedon $\mathrm{sp}$. (Figure 137). Zamamidines A (742) and B (743) exhibit cytotoxic activity against P388 murine leukemia (IC50 13.8 and $14.8 \mu \mathrm{g} / \mathrm{mL}$, respectively), but not against $\mathrm{KB}$ human epidermoid carcinoma cells. Zamamidine C (744), 3,4-dihydro-6-hydroxy-10,11-epoxymanzamine A (745), and 3,4-dihydromanzamine $\mathrm{J} N$-oxide (746) displayed cytotoxic effects towards P388, L1210 and KB cell lines. Zamamidines 342-344 and 3,4-dihydromanzamine J $N$-oxide (746) exhibited antitrypanosomal activity against Trypanosoma brucei brucei and antimalarial activity against Plasmodium falciparum [317,318].

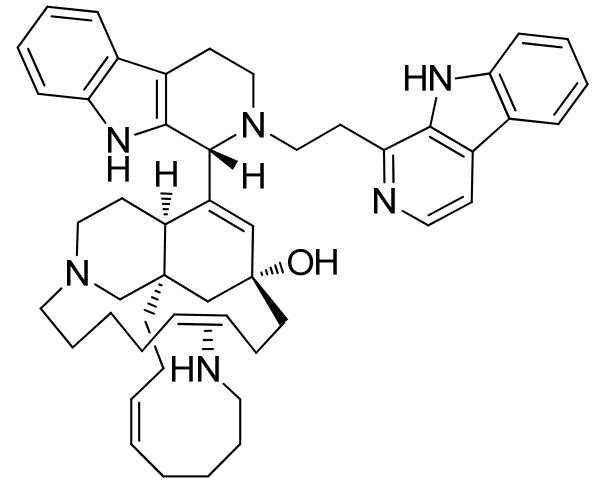

(742) zamamidine A

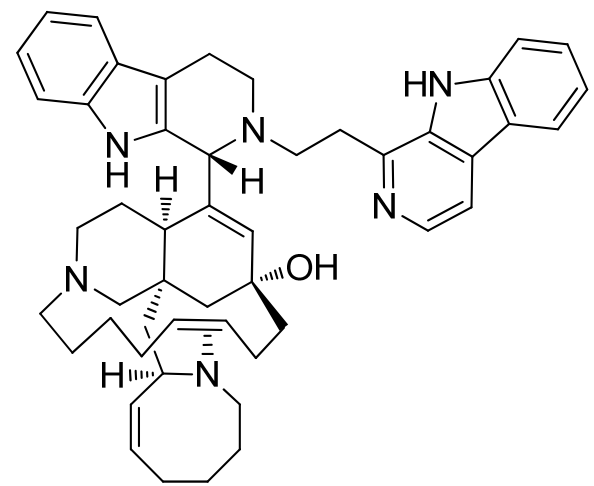

(744) zamamidine C

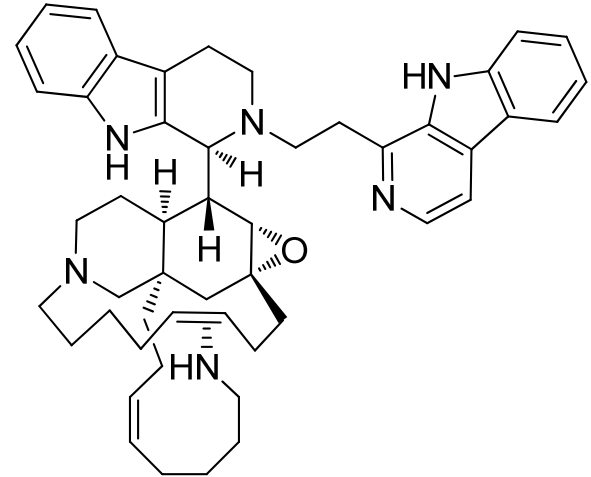

(743) zamamidine B

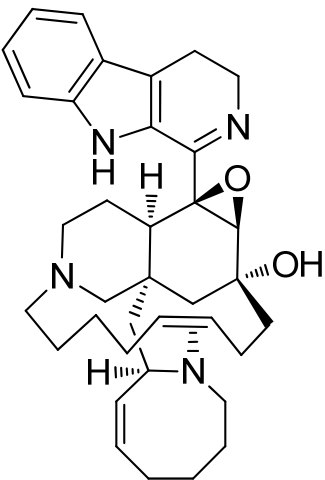

(745)

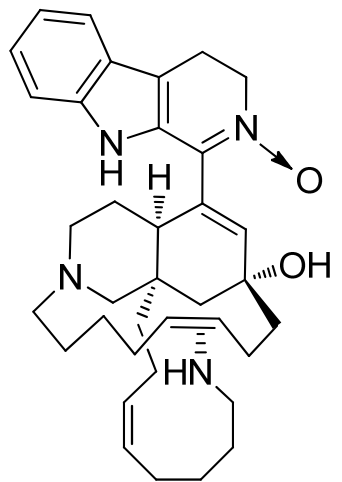

(746) 3,4-dihydromanzamine $\mathrm{J}$ $N$-oxide

Figure 137. Zamamidines $\mathrm{A}-\mathrm{C}$ and manzamine derivatives.

Acantholactone (747) was isolated from Acanthostrongylophora sp. and bears $\delta$-lactone and $\varepsilon$-lactam rings (Figure 138) [319]. Acantholactam (748) and pre-neo-kauluamine (749) were obtained from the marine sponge Acanthostrongylophora ingens. Acantholactam (748) exhibited inhibitory effects against the accumulation of cholesterol esters in macrophages, whereas pre-neo-kauluamine (749) showed proteasome inhibitory activity [320]. Further manzamine derivatives, acanthomanzamines A-E, were obtained A. ingens. Acanthomanzamines $\mathrm{A}$ and $\mathrm{B}$ possess tetrahydroisoquinoline moieties and only acanthomanzamines $\mathrm{C}-\mathrm{E}(\mathbf{7 5 0}-\mathbf{7 5 2})$ are indole-derived. Acanthomanzamines were found to have cytotoxic and inhibitory activity on accumulation of cholesterol ester in macrophages [321]. 


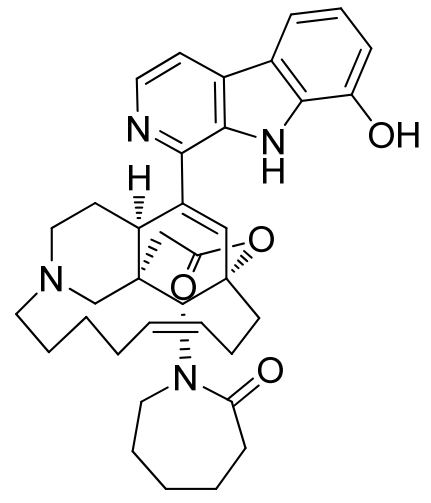

(747) acantholactone

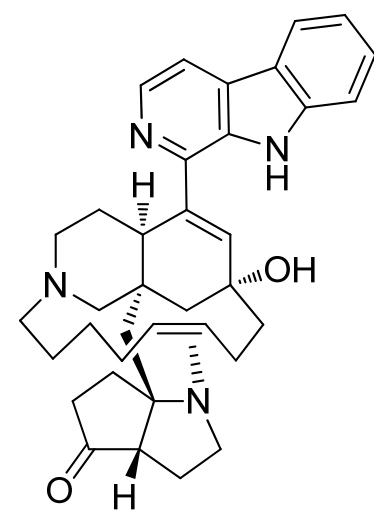

(750) acanthomanzamine C

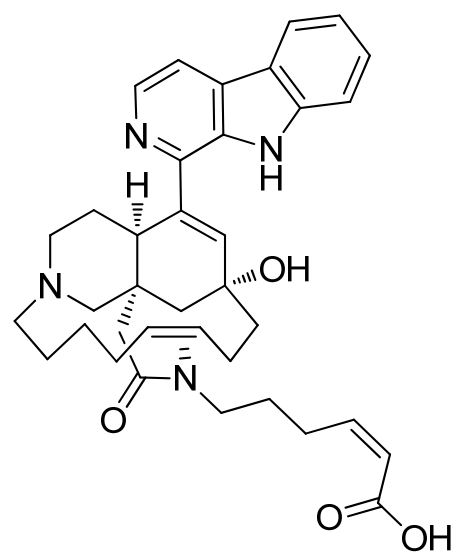

(748) acantholactam

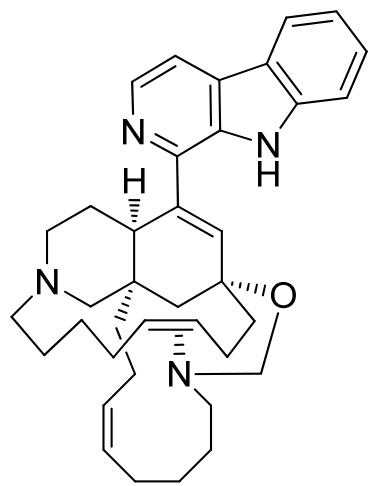

(751) acanthomanzamine D

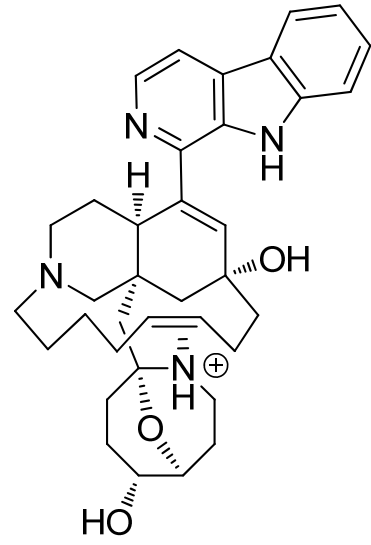

(749) pre-neo-kauluamine

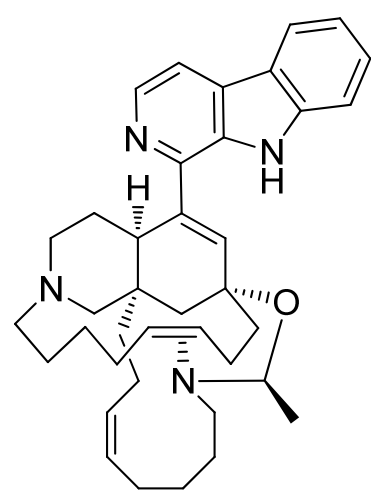

(752) acanthomanzamine $E$

Figure 138. Acantholactone, acantholactam, pre-neo-kauluamine and acanthomanzamines C-E.

Dragmacidonamines A (753) and B (754) were obtained from the sponge Dragmacidon sp. Dragmacidonamine A (753) exhibited cytotoxicity against cell line L5178Y (Figure 139) [322]. Gesashidine A (755) was isolated from the Okinawan marine sponge Thorectidae sp. (SS-1035) and showed antibacterial activity against Micrococcus luteus, but no cytotoxicity [323].<smiles>[R]C(c1nc(C(=O)O)cc2c1[nH]c1ccc(O)cc12)c1c(SC)[n+](C)cn1C</smiles>

(753) $\mathrm{R}=\mathrm{O}$ dragmacidonamines $\mathrm{A}$

(754) $\mathrm{R}=\mathrm{H}_{2}$ dragmacidonamines $\mathrm{B}$<smiles>CSc1c(Cc2nccc3c2[nH]c2ccc(O)cc23)n(C)c[n+]1C</smiles>

(755) gesashidine A

Figure 139. Dragmacidonamines $A$ and $B$ and gesashidine $A$.

Acanthomine A (756) was obtained from the marine sponge Acanthostrongylophora ingens (Figure 140) [324]. 5-Bromo-8-methoxy-1-methyl- $\beta$-carboline (757) was isolated from the New Zealand marine bryozoan Pterocella vesiculosa and displayed cytotoxicity against the P388 murine 
leukemia cell line and inhibitory activity against Bacillus subtilis, Candida albicans and Trichophyton mentagrophytes [325].<smiles>Nc1nccc(C2=NCCc3c2[nH]c2ccccc32)n1</smiles>

(756) acanthomine A<smiles>COc1ccc(Br)c2c1[nH]c1c(C)nccc12</smiles>

(757) 5-bromo-8-methoxy-1-methyl- $\beta$-carboline

Figure 140. Acanthomine $A$ and 5-bromo-8-methoxy-1-methyl- $\beta$-carboline.

Eudistomins $\mathrm{Y}_{1}-\mathrm{Y}_{7}$ (758-764) were isolated from the marine tunicate Eudistoma sp. (Korea) and revealed antibacterial activity against Staphylococcus epidermis and Bacillus subtilis (Figure 141) [326]. Eudistomidins G-K (766-770) were obtained from the Okinawan tunicate Eudistoma glaucus and the structure of eudistomidin B (765) was revised. Eudistomidins G (766) and B (765) showed cytotoxic activity towards murine leukemia cells L1210 (IC50 4.8 and $4.7 \mu \mathrm{g} / \mathrm{mL}$, respectively), whereas eudistomidin J (769) was active against murine leukemia cells P388 (IC50, $0.043 \mu \mathrm{g} / \mathrm{mL}$ ) and L1210 ( $\mathrm{IC}_{50} 0.047 \mu \mathrm{g} / \mathrm{mL}$ ) and human epidermoid carcinoma cells $\mathrm{KB}\left(\mathrm{IC}_{50} 0.063 \mu \mathrm{g} / \mathrm{mL}\right.$ ) [327,328].<smiles>[R]c1cc2[nH]c3c(C(=O)c4cc([R4])c(O)c([R])c4)nccc3c2cc1[R]</smiles>

(758) $\mathrm{R}^{1,2,3,4}=\mathrm{H}$ eudistomin $Y_{1}$ (759) $\mathrm{R}^{1}=\mathrm{Br}, \mathrm{R}^{2,3,4}=\mathrm{H}$ eudistomin $\mathrm{Y}_{2}$ (760) $\mathrm{R}^{1,2,4}=\mathrm{H}, \mathrm{R}^{2}=\mathrm{Br}$ eudistomin $\mathrm{Y}_{3}$ (761) $\mathrm{R}^{1,3}=\mathrm{Br}, \mathrm{R}^{2,4}=\mathrm{H}$ eudistomin $\mathrm{Y}_{4}$ (762) $\mathrm{R}^{1,2}=\mathrm{H}, \mathrm{R}^{3,4}=\mathrm{Br}$ eudistomin $\mathrm{Y}_{5}$ (763) $R^{1,3,4}=\mathrm{Br}, \mathrm{R}^{2}=\mathrm{H}$ eudistomin $Y_{6}$ (764) $\mathrm{R}^{1}=\mathrm{H}, \mathrm{R}^{2,3,4}=\mathrm{Br} \quad$ eudistomin $\mathrm{Y}_{7}$<smiles>[R]c1cc2[nH]c3c(c2cc1[R])CCN(C)[C@H]3[C@H](Cc1ccccc1)NC</smiles><smiles>[R]c1cc2c3c4n(c2cc1[R])CN(C)[C@H](Cc1ccccc1)[C@H]4N(C)CC3</smiles>

(765) $\mathrm{R}^{1}=\mathrm{Br}, \mathrm{R}^{2}=\mathrm{H}$ eudistomidin $\mathrm{B} \quad(\mathbf{7 6 7}) \mathrm{R}^{1}=\mathrm{H}, \mathrm{R}^{2}=\mathrm{Br}$ eudistomidin $\mathrm{H}$ (766) $\mathrm{R}^{1}=\mathrm{H}, \mathrm{R}^{2}=\mathrm{Br}$ eudistomidin $\mathrm{G}$ (768) $R^{1}=B r, R^{2}=H$ eudistomidin I<smiles>CNC(CS(C)=O)c1nccc2c1[nH]c1ccc(O)c(Br)c12</smiles>

(769) eudistomidin J<smiles>CC(=O)c1nccc2c1[nH]c1ccc(O)c(Br)c12</smiles>

(770) eudistomidin $\mathrm{K}$

Figure 141. Eudistomins $Y_{1}-Y_{7}$ and eudistomidins $B$ and $G-K$.

$\beta$-Carboline dimers 771-773 were isolated from an ascidian Didemnum sp. (Figure 142) [329]. 


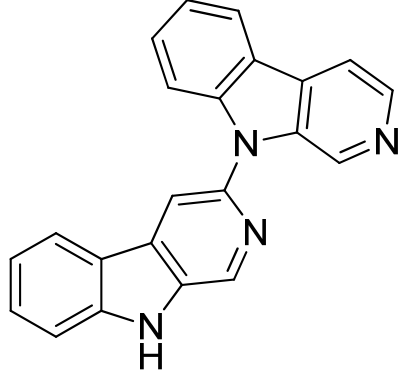

(771)

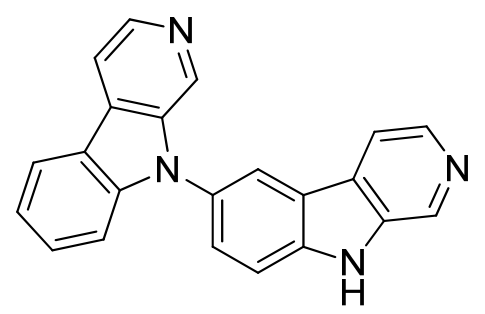

(772)

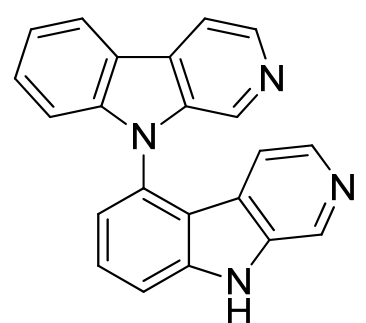

(773)

Figure 142. $\beta$-Carboline dimers.

1-Carboxy-6-hydroxy-3,4-dihydro- $\beta$-carboline (774) [330] and hyrtioerectines D-F (775-777) [331] have been isolated from the marine sponge Hyrtios sp. (Figure 143). 1-Carboxy-6-hydroxy-3,4dihydro- $\beta$-carboline (774) showed only weak activity against the isocitrate lyase (ICL) of Candida albicans (ATCC 10231) [330]. Hyrtioerectines D-F (775-777) displayed antimicrobial activity against C. albicans and Staphylococcus aureus, as well as free radical scavenging and anticancer (A549, HT-29, MDA-MB-231) activities. Diphenolic compounds (775) and (777) showed higher activities than (776) [331]. Hyrtiocarboline (778) was obtained from the marine sponge Hyrtios reticulatus (Papua New Guinea) and exhibited selective antiproliferative activity towards the tumor cell lines H522-T1, MDA-MB-435 and U937 [332]. Studies of the same organism led to the isolation of hyrtioreticulins A-F. Hyrtioreticulins A, B, E and F (779-782) possess a $\beta$-carboline framework while hyrtioreticulins $\mathrm{C}(699)$ and D (700) belong to the group of azepino-indole-type alkaloids (Figure 126). In addition, hyrtioreticulins A (780) and B (781) were found to inhibit ubiquitin-activating enzyme E1 [296,297]. 6-Oxofascaplysin (783) and secofascaplysic acid (784) were obtained from an Australian Hyrtios sp. Both exhibited low cytotoxic activity towards LNCaP and NFF cell lines [333].

Dysideanin B (785) was obtained from the marine sponge Dysidea sp. and showed antibacterial activity against Bacillus subtilis, Staphylococcus aureus, Escherichia coli and Vibrio alginolyticus (Figure 144) [334]. 2,3,5,6,11,11b-Hexahydro-2-hydroxy-1H-indolizino[8,7-b]indole-2-carboxylic acid (786) was isolated from extracts of the South China Sea gorgonian Isis minorbrachyblasta [335]. Examination of the Australian marine sponge Ancorina sp. led to discovery of (+)-7-bromotrypargine (787), which showed growth inhibitory effects towards two Plasmodium falciparum strains (Dd2 and 3D7) [336]. Opacalines A-C (788-790) and (-)-7-bromohomotrypargine (791) were obtained from the New Zealand ascidian Pseudodistoma opacum. Opacalines B (789) and C (790) showed antimalarial activity against a chloroquine-resistant strain of Plasmodium falciparum [337]. 
<smiles>O=C(O)C1=NCCc2c1[nH]c1ccc(O)cc21</smiles>

(774) 1-carboxy-6-hydroxy3,4-dihydro- $\beta$-carboline<smiles>O=C(O)c1cc2c([nH]c3ccc(O)cc32)c(C(=O)c2c[nH]cn2)n1</smiles>

(778) hyrtiocarboline<smiles>C[C@H](NCC(=O)O)c1c(O)ccc2c1C1CC(C(=O)O)N[C@H](C)C1N2</smiles>

(782) hyrtioreticulin F<smiles></smiles>

(783) 6-oxofascaplysin<smiles>[R]c1ccc2[nH]c3cnc(-c4c[nH]c5cc(O)ccc45)c([R])c3c2c1</smiles>

(775) $\mathrm{R}^{1}=\mathrm{CO}_{2} \mathrm{H}, \mathrm{R}^{2}=\mathrm{OH}$ hyrtioerectine $\mathrm{D}$ (776) $\mathrm{R}^{1}=\mathrm{CO}_{2} \mathrm{H}, \mathrm{R}^{2}=$ OMe hyrtioerectine $\mathrm{E}$ (777) $\mathrm{R}^{1}=\mathrm{CONH}_{2}, \mathrm{R}^{2}=\mathrm{OH}$ hyrtioerectine $\mathrm{F}$<smiles>[R]C1NC(C(=O)O)CC2c3cc(O)ccc3NC12</smiles>

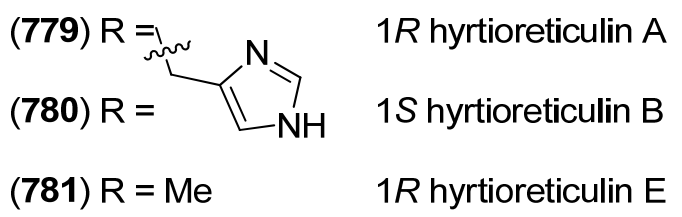<smiles>O=C(O)c1ccccc1-n1ccc2c([nH]c3ccc(O)cc32)c1=O</smiles>

(784) secofascaplysic acid

Figure 143. Carboline derivatives from Hyrtios species.<smiles>COc1ccc2[nH]c3c(N(C)C)nccc3c2c1</smiles>

(785) dysideanin B<smiles>O=C(O)[C@]1(O)C[C@H]2c3[nH]c4ccc(O)cc4c3CCN2C1</smiles>

(786)<smiles>N=C(N)NCCC[C@H]1NCCc2c1[nH]c1cc(Br)ccc21</smiles>

(787) (+)-7-bromotrypargine<smiles></smiles>

(788) $\mathrm{R}=\mathrm{H}, \mathrm{n}=2$ opacaline $\mathrm{A}$

(789) $\mathrm{R}=\mathrm{OH}, \mathrm{n}=2$ opacaline $\mathrm{B}$

(790) $\mathrm{R}=\mathrm{H}, \mathrm{n}=1$ opacaline $\mathrm{C}$<smiles>[R]n1c2c(c3ccc(Br)cc31)CC[NH2+][C@H]2CCCCNC(N)=O</smiles>

(791) (-)-7-bromohomotrypargine

Figure 144. Carboline alkaloids from Dysidea sp., Isis minorbrachyblasta, Ancorina sp., and Pseudodistoma opacum. 
Marinacarbolines A-D (792-795) together with prenylated 13- $N$-demethyl-methylpendolmycin (749) and methylpendolmycin-14-O- $\alpha$-glucoside (453, see Figure 72) were isolated from the actinomycete Marinactinospora thermotolerans SCSIO 00652 (Figure 145). They did not exhibit cytotoxic activities, but showed antiplasmodial activities against Plasmodium falciparum strains 3D7 and Dd2 [195].<smiles>[R]c1ccc(CCNC(=O)c2cc3c([nH]c4ccccc43)c(C(C)=O)n2)cc1</smiles>

(792) $\mathrm{R}=$ OMe marinacarboline $A$ (793) $\mathrm{R}=\mathrm{OH}$ marinacarboline $\mathrm{B}$ (794) $\mathrm{R}=\mathrm{H}$ marinacarboline $\mathrm{C}$<smiles>CC(=O)c1nc(C(=O)NCCc2c[nH]c3ccccc23)cc2c1[nH]c1ccccc12</smiles>

(795) marinacarboline D

Figure 145. Marinacarbolines A-D.

Examination of the actinomycete Actinomadura BCC 24717 led to the isolation of $\beta$-carbolines methyl 1-(2-methyl carbamate)ethyl- $\beta$-carboline-3-carboxylate (796), methyl 1-(propionic acid)- $\beta$ carboline-3-carboxylate (797), methyl 1 -(methyl propionate)- $\beta$-carboline-3-carboxylate (798) and 1-ethyl- $\beta$-carboline-3-carboxylic acid (799) (Figure 146). Compound 799 showed cytotoxic activity towards Vero cells [91]. Compound 797 was also isolated from Microbispora sp. LGMB259, an endophytic actinomycete isolated from Vochysia divergens. It neither displayed antibacterial or antifungal activity, nor cytotoxicity against the human cancer cell lines PC3 and A549 [338].

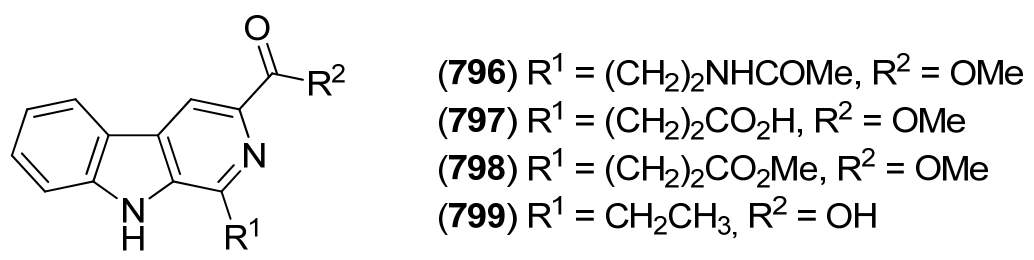

Figure 146. $\beta$-Carboline-3-carboxylates.

Hainanerectamine C (800), from the Hainan marine sponge Hyrtios erectus displays inhibitory effects on serine/threonine kinase Aurora A, which is involved in cell division regulation, yet it had no cytotoxic effects on the tumor cell lines A549 and HT-29 (Figure 147) [98].<smiles>O=C(O)[C@@H]1Cc2c([nH]c3ccc(O)cc23)[C@H](Cc2c[nH]cn2)N1</smiles>

(800) hainanerectamine C

Figure 147. Hainanerectamine C. 


\section{Conclusions}

In the twelve year period since the last review on marine indole alkaloids, the numbers of known compounds of this class has increased dramatically. Intense research, mainly driven by teams from the Asian pacific region, has provided the scientific community with new representatives of formerly known structural families but also provided entirely new chemotypes. This gain of structural knowledge is likely to trigger activities in the areas of synthetic organic chemistry, pharmacology and medicinal chemistry as the indole skeleton is the basis of many important drugs and experimental compounds in the biomedical field.

\section{Acknowledgments}

We thank the Rhineland Palatinate Natural Product Research Center and the Zeiss foundation for financial support.

\section{Conflicts of Interest}

The authors declare no conflict of interest.

\section{References}

1. Aygun, A.; Pindur, U. Chemistry and Biology of New Marine Alkaloids from the Indole and Annelated Indole Series. Curr. Med. Chem. 2003, 10, 1113-1127.

2. Pelletier, S.W. The nature and definition of an alkaloid. In Alkaloids: Chemical and Biological Perspectives; John Wiley \& Sons: New York, NY, USA, 1983.

3. Lin, Z.; Zhu, T.; Fang, Y.; Gu, Q. $1 \mathrm{H}$ and ${ }^{13} \mathrm{C}$ NMR assignments of two new indolic enamide diastereomers from a mangrove endophytic fungus Aspergillus sp. Magn. Reson. Chem. 2008, 46, 1212-1216.

4. Izumikawa, M.; Hashimoto, J.; Takagi, M.; Shin-ya, K. Isolation of two new terpeptin analogs-JBIR-81 and JBIR-82-from a seaweed-derived fungus, Aspergillus sp. SpD081030G1f1. J. Antibiot. 2010, 63, 389-391.

5. Sonnenschein, R.N.; Farias, J.J.; Tenney, K.; Mooberry, S.L.; Lobkovsky, E.; Clardy, J.; Crews, P. A Further Study of the Cytotoxic Constituents of a Milnamide-Producing Sponge. Org. Lett. 2004, 6, 779-782.

6. Chevallier, C.; Richardson, A.D.; Edler, M.C.; Hamel, E.; Harper, M.K.; Ireland, C.M. A New Cytotoxic and Tubulin-Interactive Milnamide Derivative from a Marine Sponge Cymbastela sp. Org. Lett. 2003, 5, 3737-3739.

7. Fernández, R.; Martín, M.J.; Rodríguez-Acebes, R.; Reyes, F.; Francesch, A.; Cuevas, C. Diazonamides C-E, new cytotoxic metabolites from the ascidian Diazona sp. Tetrahedron Lett. 2008, 49, 2283-2285.

8. Cho, J.Y.; Williams, P.G.; Kwon, H.C.; Jensen, P.R.; Fenical, W. Lucentamycins A-D, Cytotoxic Peptides from the Marine-Derived Actinomycete Nocardiopsis lucentensis. J. Nat. Prod. 2007, 70, 1321-1328. 
9. Sorres, J.; Martin, M.-T.; Petek, S.; Levaique, H.; Cresteil, T.; Ramos, S.; Thoison, O.; Debitus, C.; Al-Mourabit, A. Pipestelides A-C: Cyclodepsipeptides from the Pacific Marine Sponge Pipestela candelabra. J. Nat. Prod. 2012, 75, 759-763.

10. Dmitrenok, A.; Iwashita, T.; Nakajima, T.; Sakamoto, B.; Namikoshi, M.; Nagai, H. New cyclic depsipeptides from the green alga Bryopsis species; application of a carboxypeptidase hydrolysis reaction to the structure determination. Tetrahedron 2006, 62, 1301-1308.

11. Ashour, M.; Edrada, R.; Ebel, R.; Wray, V.; Wätjen, W.; Padmakumar, K.; Müller, W.E.G.; Lin, W.H.; Proksch, P. Kahalalide Derivatives from the Indian Sacoglossan Mollusk Elysia grandifolia. J. Nat. Prod. 2006, 69, 1547-1553.

12. Rao, K.V.; Na, M.; Cook, J.C.; Peng, J.; Matsumoto, R.; Hamann, M.T. Kahalalides V-Y Isolated from a Hawaiian Collection of the Sacoglossan Mollusk Elysia rufescens. J. Nat. Prod. 2008, 71, 772-778.

13. Gala, F.; D’Auria, M.V.; De Marino, S.; Sepe, V.; Zollo, F.; Smith, C.D.; Copper, J.E.; Zampella, A. Jaspamides H-L, new actin-targeting depsipeptides from the sponge Jaspis splendans. Tetrahedron 2008, 64, 7127-7130.

14. Robinson, S.J.; Morinaka, B.I.; Amagata, T.; Tenney, K.; Bray, W.M.; Gassner, N.C.; Lokey, R.S.; Crews, P. New Structures and Bioactivity Properties of Jasplakinolide (Jaspamide) Analogues from Marine Sponges. J. Med. Chem. 2010, 53, 1651-1661.

15. Li, H.; Zhang, Q.; Li, S.; Zhu, Y.; Zhang, G.; Zhang, H.; Tian, X.; Zhang, S.; Ju, J.; Zhang, C. Identification and Characterization of Xiamycin A and Oxiamycin Gene Cluster Reveals an Oxidative Cyclization Strategy Tailoring Indolosesquiterpene Biosynthesis. J. Am. Chem. Soc. 2012, 134, 8996-9005.

16. Zhang, Q.; Li, H.; Li, S.; Zhu, Y.; Zhang, G.; Zhang, H.; Zhang, W.; Shi, R.; Zhang, C. Carboxyl Formation from Methyl via Triple Hydroxylations by XiaM in Xiamycin A Biosynthesis. Org. Lett. 2012, 14, 6142-6145.

17. Xu, Z.; Baunach, M.; Ding, L.; Hertweck, C. Bacterial Synthesis of Diverse Indole Terpene Alkaloids by an Unparalleled Cyclization Sequence. Angew. Chem. Int. Ed. Engl. 2012, 51, 10293-10297.

18. Awakawa, T.; Zhang, L.; Wakimoto, T.; Hoshino, S.; Mori, T.; Ito, T.; Ishikawa, J.; Tanner, M.E.; Abe, I. A Methyltransferase Initiates Terpene Cyclization in Teleocidin B Biosynthesis. J. Am. Chem. Soc. 2014, 136, 9910-9913.

19. Ames, B.D.; Liu, X.; Walsh, C.T. Enzymatic Processing of Fumiquinazoline F: A Tandem Oxidative-Acylation Strategy for the Generation of Multicyclic Scaffolds in Fungal Indole Alkaloid Biosynthesis. Biochemistry 2010, 49, 8564-8576.

20. Kato, H.; Nakahara, T.; Yamaguchi, M.; Kagiyama, I.; Finefield, J.M.; Sunderhaus, J.D.; Sherman, D.H.; Williams, R.M.; Tsukamoto, S. Bioconversion of 6-epi-Notoamide T produces metabolites of unprecedented structures in a marine-derived Aspergillus sp. Tetrahedron Lett. 2015, 56, 247-251.

21. Kato, H.; Nakamura, Y.; Finefield, J.M.; Umaoka, H.; Nakahara, T.; Williams, R.M.; Tsukamoto, S. Study on the biosynthesis of the notoamides: Pinacol-type rearrangement of the isoprenyl unit in deoxybrevianamide E and 6-hydroxydeoxybrevianamide E. Tetrahedron Lett. 2011, 52, 6923-6926. 
22. De Sá Alves, F.R.; Barreiro, E.J.; Fraga, C.A.M. From nature to drug discovery: The indole scaffold as a "privileged structure". Mini Rev. Med. Chem. 2009, 9, 782-793.

23. Xu, W.; Gavia, D.J.; Tang, Y. Biosynthesis of fungal indole alkaloids. Nat. Prod. Rep. 2014, 31, $1474-1487$.

24. Güven, K.C.; Percot, A.; Sezik, E. Alkaloids in Marine Algae. Mar. Drugs 2010, 8, 269-284.

25. Pauletti, P.M.; Cintra, L.S.; Braguine, C.G.; Da Silva Filho, A.A.; Andrade e Silva, M.L.; Cunha, W.R.; Januário, A.H. Halogenated Indole Alkaloids from Marine Invertebrates. Mar. Drugs 2010, 8, 1526-1549.

26. Radwanski, E.R.; Last, R.L. Tryptophan biosynthesis and metabolism: Biochemical and molecular genetics. Plant Cell 1995, 7, 921-934.

27. Hu, J.-F.; Schetz, J.A.; Kelly, M.; Peng, J.-N.; Ang, K.K.H.; Flotow, H.; Leong, C.Y.; Ng, S.B.; Buss, A.D.; Wilkins, S.P.; et al. New Antiinfective and Human 5-HT2 Receptor Binding Natural and Semisynthetic Compounds from the Jamaican Sponge Smenospongia aurea. J. Nat. Prod. 2002, 65, 476-480.

28. Segraves, N.L.; Crews, P. Investigation of Brominated Tryptophan Alkaloids from Two Thorectidae Sponges: Thorectandra and Smenospongia. J. Nat. Prod. 2005, 68, 1484-1488.

29. Fahey, S.J.; Carroll, A.R. Natural products isolated from species of Halgerda Bergh, 1880 (Mollusca: Nudibranchia) and their ecological and evolutionary implications. J. Chem. Ecol. 2007, 33, 1226-1234.

30. Longeon, A.; Copp, B.R.; Quévrain, E.; Roué, M.; Kientz, B.; Cresteil, T.; Petek, S.; Debitus, C.; Bourguet-Kondracki, M.-L. Bioactive Indole Derivatives from the South Pacific Marine Sponges Rhopaloeides odorabile and Hyrtios sp. Mar. Drugs 2011, 9, 879-888.

31. Shen, S.; Liu, D.; Wei, C.; Proksch, P.; Lin, W. Purpuroines A-J, halogenated alkaloids from the sponge Iotrochota purpurea with antibiotic activity and regulation of tyrosine kinases. Bioorg. Med. Chem. 2012, 20, 6924-6928.

32. Campagnuolo, C.; Fattorusso, E.; Taglialatela-Scafati, O. Plakohypaphorines A-C, Iodine-Containing Alkaloids from the Caribbean Sponge Plakortis simplex. Eur. J. Org. Chem. 2003, 2003, 284-287.

33. Borrelli, F.; Campagnuolo, C.; Capasso, R.; Fattorusso, E.; Taglialatela-Scafati, O. Iodinated Indole Alkaloids From Plakortis simplex - New Plakohypaphorines and an Evaluation of Their Antihistamine Activity. Eur. J. Org. Chem. 2004, 2004, 3227-3232.

34. Li, Y.; Li, X.; Kim, D.; Choi, H.; Son, B. Indolyl alkaloid derivatives, $N_{\mathrm{b}}$-acetyltryptamine and oxaline from a marine-derived fungus. Arch. Pharmacal Res. 2003, 26, 21-23.

35. Sauleau, P.; Martin, M.-T.; Dau, M.-E.T.H.; Youssef, D.T.A.; Bourguet-Kondracki, M.-L. Hyrtiazepine, an Azepino-indole-Type Alkaloid from the Red Sea Marine Sponge Hyrtios erectus. J. Nat. Prod. 2006, 69, 1676-1679.

36. Ashour, M.A.; Elkhayat, E.S.; Ebel, R.; Edrada, R.; Proksch, P. Indole alkaloid from the Red Sea sponge Hyrtios erectus. Arkivoc 2007, 15, 225-231.

37. Jeong, S.-Y.; Ishida, K.; Ito, Y.; Okada, S.; Murakami, M. Bacillamide, a novel algicide from the marine bacterium, Bacillus sp. SY-1, against the harmful dinoflagellate, Cochlodinium polykrikoides. Tetrahedron Lett. 2003, 44, 8005-8007. 
38. Socha, A.M.; Long, R.A.; Rowley, D.C. Bacillamides from a Hypersaline Microbial Mat Bacterium. J. Nat. Prod. 2007, 70, 1793-1795.

39. Cohen, J.; Paul, G.; Gunasekera, S.; Longley, R.; Pomponi, S. 6-Hydroxydiscodermindole, A New Discodermindole from the Marine Sponge Discodermia polydiscus. Pharm. Biol. 2004, 42, 59-61.

40. El-Gamal, A.A.; Wang, W.-L.; Duh, C.-Y. Sulfur-Containing Polybromoindoles from the Formosan Red Alga Laurencia brongniartii. J. Nat. Prod. 2005, 68, 815-817.

41. Ji, N.-Y.; Li, X.-M.; Cui, C.-M.; Wang, B.-G. Terpenes and Polybromoindoles from the Marine Red Alga Laurencia decumbens (Rhodomelaceae). Helvetica Chimica Acta 2007, 90, 1731-1736.

42. Ji, N.-Y.; Li, X.-M.; Ding, L.-P.; Wang, B.-G. Aristolane Sesquiterpenes and Highly Brominated Indoles from the Marine Red Alga Laurencia similis (Rhodomelaceae). Helvetica Chimica Acta 2007, 90, 385-391.

43. Li, C.-S.; Li, X.-M.; Cui, C.-M.; Wang, B.-G. Brominated Metabolites from the Marine Red Alga Laurencia similis. Zeitschrift für Naturforschung B 2010, 65, 87-89.

44. Su, H.; Yuan, Z.H.; Li, J.; Guo, S.J.; Deng, L.P.; Han, L.J.; Zhu, X.B.; Shi, D.Y. Two new bromoindoles from red alga Laurencia similis. Chin. Chem. Lett. 2009, 20, 456-458.

45. Wang, R.-P.; Lin, H.-W.; Li, L.-Z.; Gao, P.-Y.; Xu, Y.; Song, S.-J. Monoindole alkaloids from a marine sponge Mycale fibrexilis. Biochem. Syst. Ecol. 2012, 43, 210-213.

46. Carroll, A.R.; Buchanan, M.S.; Edser, A.; Hyde, E.; Simpson, M.; Quinn, R.J. Dysinosins B-D, Inhibitors of Factor VIIa and Thrombin from the Australian Sponge Lamellodysidea chlorea. J. Nat. Prod. 2004, 67, 1291-1294.

47. Carroll, A.R.; Pierens, G.K.; Fechner, G.; de Almeida Leone, P.; Ngo, A.; Simpson, M.; Hyde, E.; Hooper, J.N.A.; Boström, S.-L.; Musil, D.; et al. Dysinosin A: A Novel Inhibitor of Factor VIIa and Thrombin from a New Genus and Species of Australian Sponge of the Family Dysideidae. J. Am. Chem. Soc. 2002, 124, 13340-13341.

48. Reyes, F.; Martín, R.; Fernández, R. Granulatamides A and B, Cytotoxic Tryptamine Derivatives from the Soft Coral Eunicella granulata. J. Nat. Prod. 2006, 69, 668-670.

49. Seldes, A.M.; Rodriguez Brasco, M.F.; Hernandez Franco, L.; Palermo, J.A. Identification of two meridianins from the crude extract of the tunicate Aplidium meridianum by tandem mass spectrometry. Nat. Prod. Res. 2007, 21, 555-563.

50. Reyes, F.; Fernández, R.; Rodríguez, A.; Francesch, A.; Taboada, S.; Ávila, C.; Cuevas, C. Aplicyanins A-F, new cytotoxic bromoindole derivatives from the marine tunicate Aplidium cyaneum. Tetrahedron 2008, 64, 5119-5123.

51. Š́íš, M.; Pla, D.; Altuna, M.; Francesch, A.; Cuevas, C.; Albericio, F.; Álvarez, M. Total Synthesis and Antiproliferative Activity Screening of ( \pm )-Aplicyanins A, B and E and Related Analogues. J. Med. Chem. 2009, 52, 6217-6223.

52. Ciminiello, P.; Dell'Aversano, C.; Fattorusso, E.; Forino, M.; Magno, S.; Ianaro, A.; Di Rosa, M. Oxazinin-1, -2 and -3-A Novel Toxic Compound and Its Analogues from the Digestive Glands of Mytilus galloprovincialis. Eur. J. Org. Chem. 2001, 2001, 49-53.

53. Ciminiello, P.; Dell'Aversano, C.; Fattorusso, C.; Fattorusso, E.; Forino, M.; Magno, S. Assignment of the absolute stereochemistry of oxazinin-1: Application of the 9-AMA shift-correlation method for $\beta$-chiral primary alcohols. Tetrahedron 2001, 57, 8189-8192. 
54. Ciminiello, P.; Dell'Aversano, C.; Fattorusso, E.; Forino, M.; Magno, S.; Santelia, F.U.; Moutsos, V.I.; Pitsinos, E.N.; Couladouros, E.A. Oxazinins from toxic mussels: Isolation of a novel oxazinin and reassignment of the C-2 configuration of oxazinin- 1 and -2 on the basis of synthetic models. Tetrahedron 2006, 62, 7738-7743.

55. Ciminiello, P.; Dell'Aversano, C.; Fattorusso, E.; Forino, M.; Grauso, L.; Santelia, F.U.; Tartaglione, L.; Moutsos, V.I.; Pitsinos, E.N.; Couladouros, E.A. Stereostructural Determination by a Synthetic and NMR-Based Approach of Three Oxazinins Isolated from Adriatic Mussels. Eur. J. Org. Chem. 2007, 2007, 5434-5439.

56. Bao, B.; Zhang, P.; Lee, Y.; Hong, J.; Lee, C.-O.; Jung, J. Monoindole Alkaloids from a Marine Sponge Spongosorites sp. Mar. Drugs 2007, 5, 31-39.

57. Santalova, E.A.; Denisenko, V.A.; Berdyshev, D.V.; Aminin, D.L.; Sanamyan, K.E. 6-Bromo-5hydroxyindolyl-3-glyoxylate from the Far Eastern Ascidian Syncarpa oviformis. Nat. Prod. Commun. 2008, 3, 1617-1620.

58. Abdjul, D.; Yamazaki, H.; Ukai, K.; Namikoshi, M. Two new indole derivatives from a marine sponge Ircinia sp. collected at Iriomote Island. J. Nat. Med. 2015, 69, 416-420.

59. Řezanka, T.; Hanuš, L.O.; Dembitsky, V.M.; Sigler, K. Identification of the Eight-Membered Heterocycles Hicksoanes A-C from the Gorgonian Subergorgia hicksoni. Eur. J. Org. Chem. 2008, 2008, 1265-1270.

60. Sjögren, M.; Göransson, U.; Johnson, A.-L.; Dahlström, M.; Andersson, R.; Bergman, J.; Jonsson, P.R.; Bohlin, L. Antifouling Activity of Brominated Cyclopeptides from the Marine Sponge Geodia barretti. J. Nat. Prod. 2004, 67, 368-372.

61. Hedner, E.; Sjögren, M.; Hodzic, S.; Andersson, R.; Göransson, U.; Jonsson, P.R.; Bohlin, L. Antifouling Activity of a Dibrominated Cyclopeptide from the Marine Sponge Geodia barretti. J. Nat. Prod. 2008, 71, 330-333.

62. Hawas, U.W.; Shaaban, M.; Shaaban, K.A.; Speitling, M.; Maier, A.; Kelter, G.; Fiebig, H.H.; Meiners, M.; Helmke, E.; Laatsch, H. Mansouramycins A-D, Cytotoxic Isoquinolinequinones from a Marine Streptomycete. J. Nat. Prod. 2009, 72, 2120-2124.

63. Sugiyama, Y.; Ito, Y.; Suzuki, M.; Hirota, A. Indole Derivatives from a Marine Sponge-Derived Yeast as DPPH Radical Scavengers. J. Nat. Prod. 2009, 72, 2069-2071.

64. Qi, S.-H.; Wang, Y.-F.; Zhang, S. Steroids and alkaloids from the South China Sea sponge Axinella sp. J. Asian. Nat. Prod. Res. 2009, 11, 1040-1044.

65. Capon, R.J.; Peng, C.; Dooms, C. Trachycladindoles A-G: Cytotoxic heterocycles from an Australian marine sponge, Trachycladus laevispirulifer. Org. Biomol. Chem. 2008, 6, 2765-2771.

66. Al-Zereini, W.; Fotso Fondja Yao, C.B.; Laatsch, H.; Anke, H. Aqabamycins A-G: Novel nitro maleimides from a marine Vibrio species. I. Taxonomy, fermentation, isolation and biological activities. J. Antibiot. 2010, 63, 297-301.

67. Fotso Fondja Yao, C.B.; Zereini, W.A.; Fotso, S.; Anke, H.; Laatsch, H. Aqabamycins A-G: Novel nitro maleimides from a marine Vibrio species: II. Structure elucidation. J. Antibiot. 2010, 63, 303-308. 
68. Carbone, M.; Li, Y.; Irace, C.; Mollo, E.; Castelluccio, F.; Di Pascale, A.; Cimino, G.; Santamaria, R.; Guo, Y.-W.; Gavagnin, M. Structure and Cytotoxicity of Phidianidines A and B: First Finding of 1,2,4-Oxadiazole System in a Marine Natural Product. Org. Lett. 2011, 13, 2516-2519.

69. Vitale, R.M.; Gatti, M.; Carbone, M.; Barbieri, F.; Felicità, V.; Gavagnin, M.; Florio, T.; Amodeo, P. Minimalist Hybrid Ligand/Receptor-Based Pharmacophore Model for CXCR4 Applied to a Small-Library of Marine Natural Products Led to the Identification of Phidianidine A as a New CXCR4 Ligand Exhibiting Antagonist Activity. ACS Chem. Biol. 2013, 8, 2762-2770.

70. Brogan, J.T.; Stoops, S.L.; Lindsley, C.W. Total synthesis and biological evaluation of phidianidines $\mathrm{A}$ and $\mathrm{B}$ uncovers unique pharmacological profiles at CNS targets. ACS Chem. Neurosci. 2012, 3, 658-664.

71. Jiang, C.-S.; Fu, Y.; Zhang, L.; Gong, J.-X.; Wang, Z.-Z.; Xiao, W.; Zhang, H.-Y.; Guo, Y.-W. Synthesis and biological evaluation of novel marine-derived indole-based 1,2,4-oxadiazoles derivatives as multifunctional neuroprotective agents. Bioorg. Med. Chem. Lett. 2015, 25, 216-220.

72. Carroll, A.R.; Wild, S.J.; Duffy, S.; Avery, V.M. Kororamide A, a new tribrominated indole alkaloid from the Australian bryozoan Amathia tortuosa. Tetrahedron Lett. 2012, 53, 2873-2875.

73. Gang, C.; Shen, M.-X.; Xin, W.; Fan, X.-M.; Ma, H.-M.; Wu, H.-H.; Pei, Y.-H. Indole alkaloids from the marine bacterium Pantoea agglomerans. Chem. Nat. Compd. 2013, 49, 291-293.

74. Carroll, A.R.; Avery, V.M. Leptoclinidamines A-C, Indole Alkaloids from the Australian Ascidian Leptoclinides durus. J. Nat. Prod. 2009, 72, 696-699.

75. Yamazaki, H.; Wewengkang, D.S.; Nishikawa, T.; Rotinsulu, H.; Mangindaan, R.E.P.; Namikoshi, M. Two New Tryptamine Derivatives, Leptoclinidamide and (-)-Leptoclinidamine B, from an Indonesian Ascidian Leptoclinides dubius. Mar. Drugs 2012, 10, 349.

76. Zaharenko, A.J.; Picolo, G.; Ferreira, W.A.; Murakami, T.; Kazuma, K.; Hashimoto, M.; Cury, Y.; de Freitas, J.C.; Satake, M.; Konno, K. Bunodosine 391: An Analgesic Acylamino Acid from the Venom of the Sea Anemone Bunodosoma cangicum. J. Nat. Prod. 2011, 74, 378-382.

77. Kura, K.I.; Kubota, T.; Fromont, J.; Kobayashi, J.I. Pyrinodemins E and F, new 3-alkylpyridine alkaloids from sponge Amphimedon sp. Bioorg. Med. Chem. Lett. 2011, 21, 267-270.

78. Cui, C.-M.; Li, X.-M.; Li, C.-S.; Proksch, P.; Wang, B.-G. Cytoglobosins A-G, Cytochalasans from a Marine-Derived Endophytic Fungus, Chaetomium globosum QEN-14. J. Nat. Prod. 2010, 73, 729-733.

79. Takahashi, Y.; Kubota, T.; Shibazaki, A.; Gonoi, T.; Fromont, J.; Kobayashi, J.I. Nakijinamines C-E, New Heteroaromatic Alkaloids from the Sponge Suberites Species. Org. Lett. 2011, 13, 3016-3019.

80. Takahashi, Y.; Tanaka, N.; Kubota, T.; Ishiyama, H.; Shibazaki, A.; Gonoi, T.; Fromont, J.; Kobayashi, J.I. Heteroaromatic alkaloids, nakijinamines, from a sponge Suberites sp. Tetrahedron 2012, 68, 8545-8550.

81. Prawat, H.; Mahidol, C.; Kaweetripob, W.; Wittayalai, S.; Ruchirawat, S. Iodo-sesquiterpene hydroquinone and brominated indole alkaloids from the Thai sponge Smenospongia sp. Tetrahedron 2012, 68, 6881-6886.

82. He, F.; Han, Z.; Peng, J.; Qian, P.-Y.; Qi, S.-H. Antifouling indole alkaloids from two marine derived fungi. Nat. Prod. Commun. 2013, 8, 329-332. 
83. Peng, J.; Lin, T.; Wang, W.; Xin, Z.; Zhu, T.; Gu, Q.; Li, D. Antiviral Alkaloids Produced by the Mangrove-Derived Fungus Cladosporium sp. PJX-41. J. Nat. Prod. 2013, 76, 1133-1140.

84. Li, J.L.; Han, S.C.; Yoo, E.S.; Shin, S.; Hong, J.; Cui, Z.; Li, H.; Jung, J.H. Anti-inflammatory Amino Acid Derivatives from the Ascidian Herdmania momus. J. Nat. Prod. 2011, 74, 1792-1797.

85. Li, J.L.; Xiao, B.; Park, M.; Yoo, E.S.; Shin, S.; Hong, J.; Chung, H.Y.; Kim, H.S.; Jung, J.H. PPAR- $\gamma$ Agonistic Metabolites from the Ascidian Herdmania momus. J. Nat. Prod. 2012, 75, 2082-2087.

86. Li, J.L.; Kim, E.L.; Wang, H.; Hong, J.; Shin, S.; Lee, C.-K.; Jung, J.H. Epimeric methylsulfinyladenosine derivatives from the marine ascidian Herdmania momus. Bioorg. Med. Chem. Lett. 2013, 23, 4701-4704.

87. Finlayson, R.; Pearce, A.N.; Page, M.J.; Kaiser, M.; Bourguet-Kondracki, M.-L.; Harper, J.L.; Webb, V.L.; Copp, B.R. Didemnidines A and B, Indole Spermidine Alkaloids from the New Zealand Ascidian Didemnum sp. J. Nat. Prod. 2011, 74, 888-892.

88. Chen, L.; Tang, X.-X.; Zheng, M.; Yi, Z.-W.; Xiao, X.; Qiu, Y.-K.; Wu, Z. A novel indole alkaloid from deep-sea sediment metagenomic clone-derived Escherichia coli fermentation broth. J. Asian. Nat. Prod. Res. 2011, 13, 444-448.

89. Wang, Y.; Tang, X.; Shao, Z.; Ren, J.; Liu, D.; Proksch, P.; Lin, W. Indole-based alkaloids from deep-sea bacterium Shewanella piezotolerans with antitumor activities. J. Antibiot. 2014, 67, 395-399.

90. Murcia, C.; Coello, L.; Fernández, R.; Martín, M.; Reyes, F.; Francesch, A.; Munt, S.; Cuevas, C. Tanjungides A and B: New Antitumoral Bromoindole Derived Compounds from Diazona cf. formosa. Isolation and Total Synthesis. Mar. Drugs 2014, 12, 1116-1130.

91. Kornsakulkarn, J.; Saepua, S.; Boonruangprapa, T.; Suphothina, S.; Thongpanchang, C. New $\beta$-carboline and indole alkaloids from Actinomycete Actinomadura sp. BCC 24717. Phytochem. Lett. 2013, 6, 491-494.

92. Huang, X.-L.; Gao, Y.; Xue, D.-Q.; Liu, H.-L.; Peng, C.-S.; Zhang, F.-L.; Li, Z.-Y.; Guo, Y.-W. Streptomycindole, an Indole Alkaloid from a Marine Streptomyces sp. DA22 Associated with South China Sea Sponge Craniella australiensis. Helvetica Chimica Acta 2011, 94, 1838-1842.

93. Guella, G.; N'Diaye, I.; Fofana, M.; Mancini, I. Isolation, synthesis and photochemical properties of almazolone, a new indole alkaloid from a red alga of Senegal. Tetrahedron 2006, $62,1165-1170$.

94. Feng, Y.; Davis, R.A.; Sykes, M.L.; Avery, V.M.; Quinn, R.J. Iotrochamides A and B, antitrypanosomal compounds from the Australian marine sponge Iotrochota sp. Bioorg. Med. Chem. Lett. 2012, 22, 4873-4876.

95. Yang, X.-W.; Zhang, G.-Y.; Ying, J.-X.; Yang, B.; Zhou, X.-F.; Steinmetz, A.; Liu, Y.-H.; Wang, N. Isolation, Characterization, and Bioactivity Evaluation of 3-((6-Methylpyrazin-2yl)methyl)-1H-indole, a New Alkaloid from a Deep-Sea-Derived Actinomycete Serinicoccus profundi sp. nov. Mar. Drugs 2012, 11, 33-39. 
96. Hanssen, K.Ø.; Schuler, B.; Williams, A.J.; Demissie, T.B.; Hansen, E.; Andersen, J.H.; Svenson, J.; Blinov, K.; Repisky, M.; Mohn, F.; et al. A Combined Atomic Force Microscopy and Computational Approach for the Structural Elucidation of Breitfussin A and B: Highly Modified Halogenated Dipeptides from Thuiaria breitfussi. Angew. Chem. Int. Ed. Engl. 2012, $51,12238-12241$.

97. Lee, Y.-J.; Lee, D.-G.; Rho, H.S.; Krasokhin, V.B.; Shin, H.J.; Lee, J.S.; Lee, H.-S. Cytotoxic 5-Hydroxyindole Alkaloids from the Marine Sponge Scalarispongia sp. J. Heterocycl. Chem. 2013, 50, 1400-1404.

98. He, W.-F.; Xue, D.-Q.; Yao, L.-G.; Li, J.-Y.; Li, J.; Guo, Y.-W. Hainanerectamines A-C, Alkaloids from the Hainan Sponge Hyrtios erecta. Mar. Drugs 2014, 12, 3982-3993.

99. Higuchi, K.; Kawasaki, T. Simple indole alkaloids and those with a nonrearranged monoterpenoid unit. Nat. Prod. Rep. 2007, 24, 843-868.

100. Li, S.-M. Prenylated indole derivatives from fungi: Structure diversity, biological activities, biosynthesis and chemoenzymatic synthesis. Nat. Prod. Rep. 2010, 27, 57-78.

101. Byun, H.-G.; Zhang, H.; Mochizuki, M.; Adachi, K.; Shizuri, Y.; Lee, W.-J.; Kim, S.-K. Novel antifungal diketopiperazine from marine fungus. J. Antibiot. 2003, 56, 102-106.

102. Schultz, A.W.; Oh, D.-C.; Carney, J.R.; Williamson, R.T.; Udwary, D.W.; Jensen, P.R.; Gould, S.J.; Fenical, W.; Moore, B.S. Biosynthesis and Structures of Cyclomarins and Cyclomarazines, Prenylated Cyclic Peptides of Marine Actinobacterial Origin. J. Am. Chem. Soc. 2008, 130, 4507-4516.

103. Motohashi, K.; Irie, K.; Toda, T.; Matsuo, Y.; Kasai, H.; Sue, M.; Furihata, K.; Seto, H. Studies on Terpenoids Produced by Actinomycetes. J. Antibiot. 2008, 61, 75-80.

104. Devi, P.; Rodrigues, C.; Naik, C.G.; D’Souza, L. Isolation and Characterization of Antibacterial Compound from a Mangrove-Endophytic Fungus, Penicillium chrysogenum MTCC 5108. Indian J. Microbiol. 2012, 52, 617-623.

105. Zhang, P.; Meng, L.-H.; Mándi, A.; Kurtán, T.; Li, X.-M.; Liu, Y.; Li, X.; Li, C.-S.; Wang, B.-G. Brocaeloids A-C, 4-Oxoquinoline and Indole Alkaloids with C-2 Reversed Prenylation from the Mangrove-Derived Endophytic Fungus Penicillium brocae. Eur. J. Org. Chem. 2014, 2014, 4029-4036.

106. Li, C.-S.; Li, X.-M.; An, C.-Y.; Wang, B.-G. Prenylated Indole Alkaloid Derivatives from Marine Sediment-Derived Fungus Penicillium paneum SD-44. Helvetica Chimica Acta 2014, 97, $1440-1444$.

107. Zhou, Y.; Debbab, A.; Wray, V.; Lin, W.; Schulz, B.; Trepos, R.; Pile, C.; Hellio, C.; Proksch, P.; Aly, A.H. Marine bacterial inhibitors from the sponge-derived fungus Aspergillus sp. Tetrahedron Lett. 2014, 55, 2789-2792.

108. Carle, J.S.; Christophersen, C. Bromo-substituted physostigmine alkaloids from a marine bryozoa Flustra foliacea. J. Am. Chem. Soc. 1979, 101, 4012-4013.

109. Carle, J.S.; Christophersen, C. Marine alkaloids. 2. Bromo alkaloids from a marine bryozoan Flustra foliacea. Isolation and structure elucidation. J. Org. Chem. 1980, 45, 1586-1589.

110. Carle, J.S.; Christophersen, C. Marine alkaloids. 3. Bromo-substituted alkaloids from the marine bryozoan Flustra foliacea, flustramine C and flustraminol A and B. J. Org. Chem. 1981, 46, 3440-3443. 
111. Adla, S.K.; Sasse, F.; Kelter, G.; Fiebig, H.-H.; Lindel, T. Doubly prenylated tryptamines: Cytotoxicity, antimicrobial activity and cyclisation to the marine natural product flustramine A. Org. Biomol. Chem. 2013, 11, 6119-6130.

112. Sjöblom, T.; Bohlin, L.; Christophersen, C. Studies of Swedish marine organisms. II. Muscle-relaxant alkaloids from the marine bryozoan Flustra foliacea. Acta Pharm. Suec. 1983, 20, 415-418.

113. Rivera-Becerril, E.; Joseph-Nathan, P.; Pérez-Álvarez, V.M.; Morales-Ríos, M.S. Synthesis and Biological Evaluation of (-)- and (+)-Debromoflustramine B and Its Analogues as Selective Butyrylcholinesterase Inhibitors. J. Med. Chem. 2008, 51, 5271-5284.

114. Peters, L.; König, G.M.; Terlau, H.; Wright, A.D. Four New Bromotryptamine Derivatives from the Marine Bryozoan Flustra foliacea. J. Nat. Prod. 2002, 65, 1633-1637.

115. Peters, L.; Wright, A.D.; Kehraus, S.; Gündisch, D.; Tilotta, M.C.; König, G.M. Prenylated Indole Alkaloids from Flustra foliacea with Subtype Specific Binding on NAChRs. Planta. Med. 2004, 70, 883-886.

116. Bunders, C.A.; Minvielle, M.J.; Worthington, R.J.; Ortiz, M.; Cavanagh, J.; Melander, C. Intercepting Bacterial Indole Signaling with Flustramine Derivatives. J. Am. Chem. Soc. 2011, 133, 20160-20163.

117. Sala, F.; Mulet, J.; Reddy, K.P.; Bernal, J.A.; Wikman, P.; Valor, L.M.; Peters, L.; König, G.M.; Criado, M.; Sala, S. Potentiation of human $\alpha 4 \beta 2$ neuronal nicotinic receptors by a Flustra foliacea metabolite. Neurosci. Lett. 2005, 373, 144-149.

118. Rochfort, S.J.; Moore, S.; Craft, C.; Martin, N.H.; Van Wagoner, R.M.; Wright, J.L.C. Further Studies on the Chemistry of the Flustra Alkaloids from the Bryozoan Flustra foliacea. J. Nat. Prod. 2009, 72, 1773-1781.

119. Williams, R.M.; Cox, R.J. Paraherquamides, Brevianamides, and Asperparalines: Laboratory Synthesis and Biosynthesis. An Interim Report. Acc. Chem. Res. 2003, 36, 127-139.

120. Ding, Y.; Wet, J.R.D.; Cavalcoli, J.; Li, S.; Greshock, T.J.; Miller, K.A.; Finefield, J.M.; Sunderhaus, J.D.; McAfoos, T.J.; Tsukamoto, S.; et al. Genome-Based Characterization of Two Prenylation Steps in the Assembly of the Stephacidin and Notoamide Anticancer Agents in a Marine-Derived Aspergillus sp. J. Am. Chem. Soc. 2010, 132, 12733-12740.

121. Qian-Cutrone, J.; Huang, S.; Shu, Y.-Z.; Vyas, D.; Fairchild, C.; Menendez, A.; Krampitz, K.; Dalterio, R.; Klohr, S.E.; Gao, Q. Stephacidin A and B: Two Structurally Novel, Selective Inhibitors of the Testosterone-Dependent Prostate LNCaP Cells. J. Am. Chem. Soc. 2002, 124, 14556-14557.

122. Whyte, A.C.; Gloer, J.B.; Wicklow, D.T.; Dowd, P.F. Sclerotiamide: A New Member of the Paraherquamide Class with Potent Antiinsectan Activity from the Sclerotia of Aspergillus sclerotiorum. J. Nat. Prod. 1996, 59, 1093-1095.

123. Liesch, J.M.; Wichmann, C.F. Novel Antinematodal and Antiparasitic Agents from Penicillium charlesii. II. Structure Determination of Paraherquamides B, C, D, E, F, and G. J. Antibiot. 1990, 43, 1380-1386.

124. Blanchflower, S.E.; Banks, R.M.; Everett, J.R.; Manger, B.R.; Reading, C. New Paraherquamide Antibiotics with Anthelmintic Activity. J. Antibiot. 1991, 44, 492-497. 
125. Kato, H.; Yoshida, T.; Tokue, T.; Nojiri, Y.; Hirota, H.; Ohta, T.; Williams, R.M.; Tsukamoto, S. Notoamides A-D: Prenylated Indole Alkaloids Isolated from a Marine-Derived Fungus, Aspergillus sp. Angew. Chem. Int. Ed. Engl. 2007, 46, 2254-2256; Corrigendum in 2013, 52, 7909.

126. Tsukamoto, S.; Kato, H.; Greshock, T.J.; Hirota, H.; Ohta, T.; Williams, R.M. Isolation of Notoamide E, a Key Precursor in the Biosynthesis of Prenylated Indole Alkaloids in a MarineDerived Fungus, Aspergillus sp. J. Am. Chem. Soc. 2009, 131, 3834-3835.

127. Tsukamoto, S.; Kato, H.; Samizo, M.; Nojiri, Y.; Onuki, H.; Hirota, H.; Ohta, T. Notoamides F-K, Prenylated Indole Alkaloids Isolated from a Marine-Derived Aspergillus sp. J. Nat. Prod. 2008, 71, 2064-2067.

128. Tsukamoto, S.; Kawabata, T.; Kato, H.; Greshock, T.J.; Hirota, H.; Ohta, T.; Williams, R.M. Isolation of Antipodal (-)-Versicolamide B and Notoamides $\mathrm{L}-\mathrm{N}$ from a Marine-Derived Aspergillus sp. Org. Lett. 2009, 11, 1297-1300.

129. Tsukamoto, S.; Umaoka, H.; Yoshikawa, K.; Ikeda, T.; Hirota, H. Notoamide O, a Structurally Unprecedented Prenylated Indole Alkaloid, and Notoamides P-R from a Marine-Derived Fungus, Aspergillus sp. J. Nat. Prod. 2010, 73, 1438-1440.

130. Chen, M.; Shao, C.-L.; Fu, X.-M.; Xu, R.-F.; Zheng, J.-J.; Zhao, D.-L.; She, Z.-G.; Wang, C.-Y. Bioactive Indole Alkaloids and Phenyl Ether Derivatives from a Marine-Derived Aspergillus sp. Fungus. J. Nat. Prod. 2013, 76, 547-553; Corrigendum in 2013, 76, 1229.

131. Li, S.; Finefield, J.M.; Sunderhaus, J.D.; McAfoos, T.J.; Williams, R.M.; Sherman, D.H. Biochemical Characterization of NotB as an FAD-Dependent Oxidase in the Biosynthesis of Notoamide Indole Alkaloids. J. Am. Chem. Soc. 2012, 134, 788-791.

132. Tsukamoto, S.; Umaoka, H.; Yoshikawa, K.; Ikeda, T.; Hirota, H. Correction to Notoamide O, a Structurally Unprecedented Prenylated Indole Alkaloid, and Notoamides P-R from a MarineDerived Fungus, Aspergillus sp. J. Nat. Prod. 2013, 76, 1232-1232.

133. Finefield, J.M.; Williams, R.M. Correction to Synthesis of Notoamide J: A Potentially Pivotal Intermediate in the Biosynthesis of Several Prenylated Indole Alkaloids. J. Org. Chem. 2013, 78, 8214-8214.

134. Zhang, P.; Li, X.-M.; Wang, J.-N.; Li, X.; Wang, B.-G. Prenylated indole alkaloids from the marine-derived fungus Paecilomyces variotii. Chin. Chem. Lett. 2015, 26, 313-316.

135. Zhang, X.-Y.; Xu, X.-Y.; Peng, J.; Ma, C.-F.; Nong, X.-H.; Bao, J.; Zhang, G.-Z.; Qi, S.-H. Antifouling potentials of eight deep-sea-derived fungi from the South China Sea. J. Ind. Microbiol. Biotechnol. 2014, 41, 741-748.

136. Kato, H.; Nakahara, T.; Sugimoto, K.; Matsuo, K.; Kagiyama, I.; Frisvad, J.C.; Sherman, D.H.; Williams, R.M.; Tsukamoto, S. Isolation of Notoamide S and Enantiomeric 6-epi-Stephacidin A from the Fungus Aspergillus amoenus: Biogenetic Implications. Org. Lett. 2015, 17, 700-703.

137. Sunderhaus, J.D.; McAfoos, T.J.; Finefield, J.M.; Kato, H.; Li, S.; Tsukamoto, S.; Sherman, D.H.; Williams, R.M. Synthesis and Bioconversions of Notoamide T: A Biosynthetic Precursor to Stephacidin A and Notoamide B. Org. Lett. 2013, 15, 22-25. 
138. Li, S.; Srinivasan, K.; Tran, H.; Yu, F.; Finefield, J.M.; Sunderhaus, J.D.; McAfoos, T.J.; Tsukamoto, S.; Williams, R.M.; Sherman, D.H. Comparative analysis of the biosynthetic systems for fungal bicyclo[2.2.2]diazaoctane indole alkaloids: The $(+) /(-)$-notoamide, paraherquamide and malbrancheamide pathways. Med. Chem. Comm. 2012, 3, 987-996.

139. Cai, S.; Luan, Y.; Kong, X.; Zhu, T.; Gu, Q.; Li, D. Isolation and Photoinduced Conversion of 6-epi-Stephacidins from Aspergillus taichungensis. Org. Lett. 2013, 15, 2168-2171.

140. Watts, K.R.; Loveridge, S.T.; Tenney, K.; Media, J.; Valeriote, F.A.; Crews, P. Utilizing DART Mass Spectrometry to Pinpoint Halogenated Metabolites from a Marine Invertebrate-Derived Fungus. J. Org. Chem. 2011, 76, 6201-6208.

141. Peng, J.; Zhang, X.-Y.; Tu, Z.-C.; Xu, X.-Y.; Qi, S.-H. Alkaloids from the Deep-Sea-Derived Fungus Aspergillus westerdijkiae DFFSCS013. J. Nat. Prod. 2013, 76, 983-987.

142. Yang, B.; Dong, J.; Lin, X.; Zhou, X.; Zhang, Y.; Liu, Y. New prenylated indole alkaloids from fungus Penicillium sp. derived of mangrove soil sample. Tetrahedron 2014, 70, 3859-3863.

143. Wang, F.; Fang, Y.; Zhu, T.; Zhang, M.; Lin, A.; Gu, Q.; Zhu, W. Seven new prenylated indole diketopiperazine alkaloids from holothurian-derived fungus Aspergillus fumigatus. Tetrahedron 2008, 64, 7986-7991.

144. Afiyatullov, S.S.; Zhuravleva, O.; Chaikina, E.; Anisimov, M. A new spirotryprostatin from the marine isolate of the fungus Aspergillus fumigatus. Chem. Nat. Compd. 2012, 48, 95-98.

145. Zhang, M.; Wang, W.-L.; Fang, Y.-C.; Zhu, T.-J.; Gu, Q.-Q.; Zhu, W.-M. Cytotoxic Alkaloids and Antibiotic Nordammarane Triterpenoids from the Marine-Derived Fungus Aspergillus sydowi. J. Nat. Prod. 2008, 71, 985-989.

146. He, F.; Sun, Y.-L.; Liu, K.-S.; Zhang, X.-Y.; Qian, P.-Y.; Wang, Y.-F.; Qi, S.-H. Indole alkaloids from marine-derived fungus Aspergillus sydowii SCSIO 00305. J. Antibiot. 2012, 65, 109-111.

147. Wang, Y.; Li, Z.-L.; Bai, J.; Zhang, L.-M.; Wu, X.; Zhang, L.; Pei, Y.-H.; Jing, Y.-K.; Hua, H.-M. 2,5-Diketopiperazines from the Marine-Derived Fungus Aspergillus fumigatus YK-7. Chem. Biodiversity 2012, 9, 385-393.

148. An, C.-Y.; Li, X.-M.; Li, C.-S.; Xu, G.-M.; Wang, B.-G. Prenylated Indolediketopiperazine Peroxides and Related Homologues from the Marine Sediment-Derived Fungus Penicillium brefeldianum SD-273. Mar. Drugs 2014, 12, 746-756.

149. Zhuravleva, O.I.; Afiyatullov, S.S.; Denisenko, V.A.; Ermakova, S.P.; Slinkina, N.N.; Dmitrenok, P.S.; Kim, N.Y. Secondary metabolites from a marine-derived fungus Aspergillus carneus Blochwitz. Phytochemistry 2012, 80, 123-131.

150. Peng, J.; Gao, H.; Li, J.; Ai, J.; Geng, M.; Zhang, G.; Zhu, T.; Gu, Q.; Li, D. Prenylated Indole Diketopiperazines from the Marine-Derived Fungus Aspergillus versicolor. J. Org. Chem. 2014, 79, 7895-7904.

151. Song, F.; Liu, X.; Guo, H.; Ren, B.; Chen, C.; Piggott, A.M.; Yu, K.; Gao, H.; Wang, Q.; Liu, M.; et al. Brevianamides with Antitubercular Potential from a Marine-Derived Isolate of Aspergillus versicolor. Org. Lett. 2012, 14, 4770-4773.

152. Miao, F.-P.; Li, X.-D.; Liu, X.-H.; Cichewicz, R.H.; Ji, N.-Y. Secondary Metabolites from an Algicolous Aspergillus versicolor Strain. Mar. Drugs 2012, 10, 131-139. 
153. Kong, X.; Cai, S.; Zhu, T.; Gu, Q.; Li, D.; Luan, Y. Secondary metabolites of a deep sea derived fungus Aspergillus versicolor CXCTD-06-6a and their bioactivity. J. Ocean Univ. China 2014, 13, 691-695.

154. Wang, W.-L.; Lu, Z.-Y.; Tao, H.-W.; Zhu, T.-J.; Fang, Y.-C.; Gu, Q.-Q.; Zhu, W.-M. Isoechinulin-type Alkaloids, Variecolorins A-L, from Halotolerant Aspergillus variecolor. J. Nat. Prod. 2007, 70, 1558-1564.

155. Zhou, L.-N.; Zhu, T.-J.; Cai, S.-X.; Gu, Q.-Q.; Li, D.-H. Three New Indole-Containing Diketopiperazine Alkaloids from a Deep-Ocean Sediment Derived Fungus Penicillium griseofulvum. Helvetica Chimica Acta 2010, 93, 1758-1763.

156. Du, F.-Y.; Li, X.-M.; Li, C.-S.; Shang, Z.; Wang, B.-G. Cristatumins A-D, new indole alkaloids from the marine-derived endophytic fungus Eurotium cristatum EN-220. Bioorg. Med. Chem. Lett. 2012, 22, 4650-4653.

157. Wang, W.-L.; Zhu, T.-J.; Tao, H.-W.; Lu, Z.-Y.; Fang, Y.-C.; Gu, Q.-Q.; Zhu, W.-M. Three Novel, Structurally Unique Spirocyclic Alkaloids from the Halotolerant B-17 Fungal Strain of Aspergillus variecolor. Chem. Biodiversity 2007, 4, 2913-2919.

158. Li, D.-L.; Li, X.-M.; Proksch, P.; Wang, B.-G. 7-O-Methylvariecolortide A, a new spirocyclic diketopiperazine alkaloid from a marine mangrove derived endophytic fungus, Eurotium rubrum. Nat. Prod. Commun. 2010, 5, 1583-1586.

159. Chen, G.-D.; Bao, Y.-R.; Huang, Y.-F.; Hu, D.; Li, X.-X.; Guo, L.-D.; Li, J.; Yao, X.-S.; Gao, H. Three pairs of variecolortide enantiomers from Eurotium sp. with caspase-3 inhibitory activity. Fitoterapia 2014, 92, 252-259.

160. Li, D.-L.; Li, X.-M.; Li, T.-G.; Dang, H.-Y.; Wang, B.-G. Dioxopiperazine Alkaloids Produced by the Marine Mangrove Derived Endophytic Fungus Eurotium rubrum. Helvetica Chimica Acta 2008, 91, 1888-1893.

161. Yan, H.-J.; Li, X.-M.; Li, C.-S.; Wang, B.-G. Alkaloid and Anthraquinone Derivatives Produced by the Marine-Derived Endophytic Fungus Eurotium rubrum. Helvetica Chimica Acta 2012, 95, $163-168$.

162. Chen, X.; Si, L.; Liu, D.; Proksch, P.; Zhang, L.; Zhou, D.; Lin, W. Neoechinulin B and its analogues as potential entry inhibitors of influenza viruses, targeting viral hemagglutinin. Eur. $J$. Med. Chem. 2015, 93, 182-195.

163. Meng, L.-H.; Du, F.-Y.; Li, X.-M.; Pedpradab, P.; Xu, G.-M.; Wang, B.-G. Rubrumazines A-C, Indolediketopiperazines of the Isoechinulin Class from Eurotium rubrum MA-150, a Fungus Obtained from Marine Mangrove-Derived Rhizospheric Soil. J. Nat. Prod. 2015, 78, 909-913.

164. Gao, H.; Liu, W.; Zhu, T.; Mo, X.; Mandi, A.; Kurtan, T.; Li, J.; Ai, J.; Gu, Q.; Li, D. Diketopiperazine alkaloids from a mangrove rhizosphere soil derived fungus Aspergillus effuses H1-1. Org. Biomol. Chem. 2012, 10, 9501-9506.

165. Gao, H.; Zhu, T.; Li, D.; Gu, Q.; Liu, W. Prenylated indole diketopiperazine alkaloids from a mangrove rhizosphere soil derived fungus Aspergillus effuses H1-1. Arch. Pharmacal Res. 2013, 36, 952-956.

166. Zou, X.; Li, Y.; Zhang, X.; Li, Q.; Liu, X.; Huang, Y.; Tang, T.; Zheng, S.; Wang, W.; Tang, J. A New Prenylated Indole Diketopiperazine Alkaloid from Eurotium cristatum. Molecules 2014, 19, 17839-17847. 
167. Gomes, N.M.; Dethoup, T.; Singburaudom, N.; Gales, L.; Silva, A.M.S.; Kijjoa, A. Eurocristatine, a new diketopiperazine dimer from the marine sponge-associated fungus Eurotium cristatum. Phytochemistry Letters 2012, 5, 717-720.

168. Sprogøe, K.; Manniche, S.; Larsen, T.O.; Christophersen, C. Janoxepin and brevicompanine B: Antiplasmodial metabolites from the fungus Aspergillus janus. Tetrahedron 2005, 61, 8718-8721.

169. Kusano, M.; Sotoma, G.; Koshino, H.; Uzawa, J.; Chijimatsu, M.; Fujioka, S.; Kawano, T.; Kimura, Y. Brevicompanines A and B: New plant growth regulators produced by the fungus, Penicillium brevicompactum. J. Chem. Soc., Perkin Trans. 1 1998, 2823-2826.

170. Kimura, Y.; Sawada, A.; Kuramata, M.; Kusano, M.; Fujioka, S.; Kawano, T.; Shimada, A. Brevicompanine C, Cyclo-(D-Ile-L-Trp), and Cyclo-(D-Leu-L-Trp), Plant Growth Regulators from Penicillium brevi-compactum. J. Nat. Prod. 2005, 68, 237-239.

171. Du, L.; Yang, X.; Zhu, T.; Wang, F.; Xiao, X.; Park, H.; Gu, Q. Diketopiperazine Alkaloids from a Deep Ocean Sediment Derived Fungus Penicillium sp. Chem. Pharm. Bull. 2009, 57, 873-876.

172. Raju, R.; Piggott, A.M.; Huang, X.-C.; Capon, R.J. Nocardioazines: A Novel Bridged Diketopiperazine Scaffold from a Marine-Derived Bacterium Inhibits P-Glycoprotein. Org. Lett. 2011, 13, 2770-2773.

173. Che, Q.; Zhu, T.; Qi, X.; Mándi, A.; Kurtán, T.; Mo, X.; Li, J.; Gu, Q.; Li, D. Hybrid Isoprenoids from a Reeds Rhizosphere Soil Derived Actinomycete Streptomyces sp. CHQ-64. Org. Lett. 2012, 14, 3438-3441.

174. Che, Q.; Zhu, T.; Keyzers, R.A.; Liu, X.; Li, J.; Gu, Q.; Li, D. Polycyclic Hybrid Isoprenoids from a Reed Rhizosphere Soil Derived Streptomyces sp. CHQ-64. J. Nat. Prod. 2013, 76, 759-763.

175. Cai, S.; Sun, S.; Peng, J.; Kong, X.; Zhou, H.; Zhu, T.; Gu, Q.; Li, D. Okaramines S-U, three new indole diketopiperazine alkaloids from Aspergillus taichungensis ZHN-7-07. Tetrahedron 2015, 71, 3715-3719.

176. Khalil, Z.G.; Huang, X.-C.; Raju, R.; Piggott, A.M.; Capon, R.J. Shornephine A: Structure, Chemical Stability, and P-Glycoprotein Inhibitory Properties of a Rare Diketomorpholine from an Australian Marine-Derived Aspergillus sp. J. Org. Chem. 2014, 79, 8700-8705.

177. Numata, A.; Takahashi, C.; Ito, Y.; Takada, T.; Kawai, K.; Usami, Y.; Matsumura, E.; Imachi, M.; Ito, T.; Hasegawa, T. Communesins, cytotoxic metabolites of a fungus isolated from a marine alga. Tetrahedron Lett. 1993, 34, 2355-2358.

178. Hayashi, H.; Matsumoto, H.; Akiyama, K. New Insecticidal Compounds, Communesins C, D and E, from Penicillium expansum Link MK-57. Biosci. Biotechnol. Biochem. 2004, 68, 753-756.

179. Jadulco, R.; Edrada, R.A.; Ebel, R.; Berg, A.; Schaumann, K.; Wray, V.; Steube, K.; Proksch, P. New Communesin Derivatives from the Fungus Penicillium sp. Derived from the Mediterranean Sponge Axinella verrucosa. J. Nat. Prod. 2004, 67, 78-81.

180. Dalsgaard, P.W.; Blunt, J.W.; Munro, M.H.G.; Frisvad, J.C.; Christophersen, C. Communesins G and H, New Alkaloids from the Psychrotolerant Fungus Penicillium rivulum. J. Nat. Prod. 2005, $68,258-261$.

181. Lin, H.-C.; Chiou, G.; Chooi, Y.-H.; McMahon, T.C.; Xu, W.; Garg, N.K.; Tang, Y. Elucidation of the Concise Biosynthetic Pathway of the Communesin Indole Alkaloids. Angew. Chem. Int. Ed. Engl. 2015, 127, 3047-3050. 
182. Belofsky, G.N.; Gloer, J.B.; Wicklow, D.T.; Dowd, P.F. Antiinsectan alkaloids: Shearinines A-C and a new paxilline derivative from the ascostromata of Eupenicillium shearii. Tetrahedron 1995, 51, 3959-3968.

183. Xu, M.; Gessner, G.; Groth, I.; Lange, C.; Christner, A.; Bruhn, T.; Deng, Z.; Li, X.; Heinemann, S.H.; Grabley, S.; et al. Shearinines D-K, new indole triterpenoids from an endophytic Penicillium sp. (strain HKI0459) with blocking activity on large-conductance calcium-activated potassium channels. Tetrahedron 2007, 63, 435-444.

184. Smetanina, O.F.; Kalinovsky, A.I.; Khudyakova, Y.V.; Pivkin, M.V.; Dmitrenok, P.S.; Fedorov, S.N.; Ji, H.; Kwak, J.-Y.; Kuznetsova, T.A. Indole Alkaloids Produced by a Marine Fungus Isolate of Penicillium janthinellum Biourge. J. Nat. Prod. 2007, 70, 906-909.

185. Smetanina, O.F.; Kalinovsky, A.I.; Khudyakova, Y.V.; Dmitrenok, P.S.; Federov, S.N.; Ji, H.; Kwak, J.-Y.; Kuznetsova, T.A. Indole Alkaloids Produced by a Marine Fungus Isolate of Penicillium janthinellum Biourge. J. Nat. Prod. 2007, 70, 906-909.

186. Belofsky, G.N.; Gloer, J.B.; Wicklow, D.T.; Dowd, P.F. Indole Antiinsectan Metabolites from the Ascostromata of Eupenicillium shearii. U.S. Patent 5,492,902 A, 20 February 1996.

187. You, J.; Du, L.; King, J.B.; Hall, B.E.; Cichewicz, R.H. Small-Molecule Suppressors of Candida albicans Biofilm Formation Synergistically Enhance the Antifungal Activity of Amphotericin B against Clinical Candida Isolates. ACS Chem. Biol. 2013, 8, 840-848.

188. Qiao, M.-F.; Ji, N.-Y.; Liu, X.-H.; Li, K.; Zhu, Q.-M.; Xue, Q.-Z. Indoloditerpenes from an algicolous isolate of Aspergillus oryzae. Bioorg. Med. Chem. Lett. 2010, 20, 5677-5680.

189. Tsuda, M.; Kasai, Y.; Komatsu, K.; Sone, T.; Tanaka, M.; Mikami, Y.; Kobayashi, J.I. Citrinadin A, a Novel Pentacyclic Alkaloid from Marine-Derived Fungus Penicillium citrinum. Org. Lett. 2004, 6, 3087-3089.

190. Mugishima, T.; Tsuda, M.; Kasai, Y.; Ishiyama, H.; Fukushi, E.; Kawabata, J.; Watanabe, M.; Akao, K.; Kobayashi, J.I. Absolute Stereochemistry of Citrinadins A and B from Marine-Derived Fungus. J. Org. Chem. 2005, 70, 9430-9435.

191. Nakae, K.; Hosokawa, N.; Sawa, R.; Kubota, Y.; Masuda, T.; Ohba, S.; Igarashi, M.; Nakagawa, N.; Nishimura, Y.; Akamatsu, Y. A New Teleocidin Analog from Streptomyces sp. MM216-87F4 Induces Substance P Release from Rat Dorsal Root Ganglion Neurons. J. Antibiot. 2006, 59, 11-17.

192. Izumikawa, M.; Khan, S.T.; Komaki, H.; Takagi, M.; Shin-ya, K. JBIR-31, a new teleocidin analog, produced by salt-requiring Streptomyces sp. NBRC 105896 isolated from a marine sponge. J. Antibiot. 2009, 63, 33-36.

193. Jiang, W.; Tan, S.; Hanaki, Y.; Irie, K.; Uchida, H.; Watanabe, R.; Suzuki, T.; Sakamoto, B.; Kamio, M.; Nagai, H. Two New Lyngbyatoxin Derivatives from the Cyanobacterium, Moorea producens. Mar. Drugs 2014, 12, 5788-5800.

194. Jiang, W.; Zhou, W.; Uchida, H.; Kikumori, M.; Irie, K.; Watanabe, R.; Suzuki, T.; Sakamoto, B.; Kamio, M.; Nagai, H. A New Lyngbyatoxin from the Hawaiian Cyanobacterium Moorea producens. Mar. Drugs 2014, 12, 2748-2759.

195. Huang, H.; Yao, Y.; He, Z.; Yang, T.; Ma, J.; Tian, X.; Li, Y.; Huang, C.; Chen, X.; Li, W.; et al. Antimalarial $\beta$-Carboline and Indolactam Alkaloids from Marinactinospora thermotolerans, a Deep Sea Isolate. J. Nat. Prod. 2011, 74, 2122-2127. 
196. Du, L.; Li, D.; Zhu, T.; Cai, S.; Wang, F.; Xiao, X.; Gu, Q. New alkaloids and diterpenes from a deep ocean sediment derived fungus Penicillium sp. Tetrahedron 2009, 65, 1033-1039.

197. Du, L.; Feng, T.; Zhao, B.; Li, D.; Cai, S.; Zhu, T.; Wang, F.; Xiao, X.; Gu, Q. Alkaloids from a deep ocean sediment-derived fungus Penicillium sp. and their antitumor activities. J. Antibiot. 2010, 63, 165-170.

198. Ding, L.; Münch, J.; Goerls, H.; Maier, A.; Fiebig, H.-H.; Lin, W.-H.; Hertweck, C. Xiamycin, a pentacyclic indolosesquiterpene with selective anti-HIV activity from a bacterial mangrove endophyte. Bioorg. Med. Chem. Lett. 2010, 20, 6685-6687.

199. Ding, L.; Maier, A.; Fiebig, H.-H.; Lin, W.-H.; Hertweck, C. A family of multicyclic indolosesquiterpenes from a bacterial endophyte. Org. Biomol. Chem. 2011, 9, 4029-4031.

200. Zhang, Q.; Mándi, A.; Li, S.; Chen, Y.; Zhang, W.; Tian, X.; Zhang, H.; Li, H.; Zhang, W.; Zhang, S.; et al. N-N-Coupled Indolo-sesquiterpene Atropo-Diastereomers from a Marine-Derived Actinomycete. Eur. J. Org. Chem. 2012, 2012, 5256-5262.

201. Sallam, A.A.; Houssen, W.E.; Gissendanner, C.R.; Orabi, K.Y.; Foudah, A.I.; El Sayed, K.A. Bioguided discovery and pharmacophore modeling of the mycotoxic indole diterpene alkaloids penitrems as breast cancer proliferation, migration, and invasion inhibitors. Med. Chem. Comm. 2013, 4, 1360-1369.

202. Zhang, P.; Li, X.-M.; Li, X.; Wang, B.-G. New indole-diterpenoids from the algal-associated fungus Aspergillus nidulans. Phytochem. Lett. 2015, 12, 182-185.

203. Fan, Y.; Wang, Y.; Liu, P.; Fu, P.; Zhu, T.; Wang, W.; Zhu, W. Indole-Diterpenoids with Anti-H1N1 Activity from the Aciduric Fungus Penicillium camemberti OUCMDZ-1492. J. Nat. Prod. 2013, 76, 1328-1336.

204. Harms, H.; Rempel, V.; Kehraus, S.; Kaiser, M.; Hufendiek, P.; Müller, C.E.; König, G.M. Indoloditerpenes from a Marine-Derived Fungal Strain of Dichotomomyces cejpii with Antagonistic Activity at GPR18 and Cannabinoid Receptors. J. Nat. Prod. 2014, 77, 673-677.

205. Makarieva, T.N.; Ilyin, S.G.; Stonik, V.A.; Lyssenko, K.A.; Denisenko, V.A. Pibocin, the first ergoline marine alkaloid from the Far-Eastern ascidian Eudistoma sp. Tetrahedron Lett. 1999, 40, 1591-1594.

206. Makarieva, T.N.; Dmitrenok, A.S.; Dmitrenok, P.S.; Grebnev, B.B.; Stonik, V.A. Pibocin B, the First $\mathrm{N}$-O-Methylindole Marine Alkaloid, a Metabolite from the Far-Eastern Ascidian Eudistoma Species. J. Nat. Prod. 2001, 64, 1559-1561.

207. Spilsbury, J.F.; Wilkinson, S. The isolation of festuclavine and two new clavine alkaloids from Aspergillus fumigatus Fres. J. Chem. Soc. 1961, 2081-2091, doi:10.1039/jr9610002085.

208. Cole, R.J.; Kirksey, J.W.; Dorner, J.W.; Wilson, D.M.; Johnson, J.C.; Johnson, A.N.; Bedell, D.M.; Springer, J.P.; Chexal, K.K. Mycotoxins produced by Aspergillus fumigatus species isolated from molded silage. J. Agric. Food Chem. 1977, 25, 826-830.

209. Zhang, D.; Satake, M.; Fukuzawa, S.; Sugahara, K.; Niitsu, A.; Shirai, T.; Tachibana, K. Two new indole alkaloids, 2-(3,3-dimethylprop-1-ene)-costaclavine and 2-(3,3-dimethylprop-1-ene)epicostaclavine, from the marine-derived fungus Aspergillus fumigatus. J. Nat. Med. 2012, 66, 222-226. 
210. Li, Y.-X.; Himaya, S.W.A.; Dewapriya, P.; Zhang, C.; Kim, S.-K. Fumigaclavine C from a Marine-Derived Fungus Aspergillus fumigatus Induces Apoptosis in MCF-7 Breast Cancer Cells. Mar. Drugs 2013, 11, 5063-5086.

211. Wakimoto, T.; Tan, K.C.; Abe, I. Ergot alkaloid from the sea slug Pleurobranchus forskalii. Toxicon 2013, 72, 1-4.

212. Yang, C.-G.; Huang, H.; Jiang, B. Progress in Studies of Novel Marine Bis(indole) Alkaloids. Curr. Org. Chem. 2004, 8, 1691-1720.

213. Leena, G.; Archna, T.; Prem, M.S.C. Bis and Tris Indole Alkaloids from Marine Organisms: New Leads for Drug Discovery. Curr. Med. Chem. 2007, 14, 1789-1803.

214. Veluri, R.; Oka, I.; Wagner-Döbler, I.; Laatsch, H. New Indole Alkaloids from the North Sea Bacterium Vibrio parahaemolyticus Bio2491. J. Nat. Prod. 2003, 66, 1520-1523.

215. Tsuda, M.; Takahashi, Y.; Fromont, J.; Mikami, Y.; Kobayashi, J.I. Dendridine A, a Bis-indole Alkaloid from a Marine Sponge Dictyodendrilla Species. J. Nat. Prod. 2005, 68, 1277-1278.

216. Endo, T.; Tsuda, M.; Fromont, J.; Kobayashi, J.I. Hyrtinadine A, a Bis-indole Alkaloid from a Marine Sponge. J. Nat. Prod. 2007, 70, 423-424.

217. Cai, S.-X.; Li, D.-H.; Zhu, T.-J.; Wang, F.-P.; Xiao, X.; Gu, Q.-Q. Two New Indole Alkaloids from the Marine-Derived Bacterium Aeromonas sp. CB101. Helvetica Chimica Acta 2010, 93, 791-795.

218. Yan, X.; Tang, X.-X.; Chen, L.; Yi, Z.-W.; Fang, M.-J.; Wu, Z.; Qiu, Y.-K. Two New Cytotoxic Indole Alkaloids from a Deep-Sea Sediment Derived Metagenomic Clone. Mar. Drugs 2014, 12, 2156-2163.

219. Abe, T.; Kukita, A.; Akiyama, K.; Naito, T.; Uemura, D. Isolation and Structure of a Novel Biindole Pigment Substituted with an Ethyl Group from a Metagenomic Library Derived from the Marine Sponge Halichondria okadai. Chem. Lett. 2012, 41, 728-729.

220. Li, J.L.; Huang, L.; Liu, J.; Song, Y.; Gao, J.; Jung, J.H.; Liu, Y.; Chen, G. Acetylcholinesterase inhibitory dimeric indole derivatives from the marine actinomycetes Rubrobacter radiotolerans. Fitoterapia 2015, 102, 203-207.

221. Rubnov, S.; Chevallier, C.; Thoison, O.; Debitus, C.; Laprevote, O.; Guénard, D.; Sévenet, T. Echinosulfonic acid D: An ESI MS $^{\mathrm{n}}$ evaluation of a new cytotoxic alkaloid from the New-Caledonian sponge Psammoclemma sp. Nat. Prod. Res. 2005, 19, 75-79.

222. Yamada, T.; Iwamoto, C.; Yamagaki, N.; Yamanouchi, T.; Minoura, K.; Hagishita, S.; Numata, A. Leptosins O-S, cytotoxic metabolites of a strain of Leptosphaeria sp. isolated from a marine alga. Heterocycles 2004, 63, 641-653.

223. Usami, Y.; Yamaguchi, J.; Numata, A. Gliocladins A-C and glioperazine; cytotoxic dioxo- or trioxopiperazine metabolites from a Gliocladium sp. separated from a sea hare. Heterocycles 2004, 63, 1123-1129.

224. Han, X.-X.; Cui, C.-B.; Gu, Q.-Q.; Zhu, W.-M.; Liu, H.-B.; Gu, J.-Y.; Osada, H. ZHD-0501, a novel naturally occurring staurosporine analog from Actinomadura sp. 007. Tetrahedron Lett. 2005, 46, 6137-6140.

225. Wu, S.J.; Fotso, S.; Li, F.; Qin, S.; Kelter, G.; Fiebig, H.H.; Laatsch, H. N-Carboxamidostaurosporine and Selina-4(14),7(11)-diene-8,9-diol, New Metabolites from a Marine Streptomyces sp. J. Antibiot. 2006, 59, 331-337. 
226. Reyes, F.; Fernández, R.; Rodríguez, A.; Bueno, S.; de Eguilior, C.; Francesch, A.; Cuevas, C. Cytotoxic Staurosporines from the Marine Ascidian Cystodytes solitus. J. Nat. Prod. 2008, 71, 1046-1048.

227. Jimenez, P.C.; Wilke, D.V.; Ferreira, E.G.; Takeara, R.; de Moraes, M.O.; Silveira, E.R.; da Cruz Lotufo, T.M.; Lopes, N.P.; Costa-Lotufo, L.V. Structure Elucidation and Anticancer Activity of 7-Oxostaurosporine Derivatives from the Brazilian Endemic Tunicate Eudistoma vannamei. Mar. Drugs 2012, 10, 1092-1102.

228. Fu, P.; Yang, C.; Wang, Y.; Liu, P.; Ma, Y.; Xu, L.; Su, M.; Hong, K.; Zhu, W. Streptocarbazoles A and B, Two Novel Indolocarbazoles from the Marine-Derived Actinomycete Strain Streptomyces sp. FMA. Org. Lett. 2012, 14, 2422-2425.

229. Fu, P.; Zhuang, Y.; Wang, Y.; Liu, P.; Qi, X.; Gu, K.; Zhang, D.; Zhu, W. New Indolocarbazoles from a Mutant Strain of the Marine-Derived Actinomycete Streptomyces fradiae 007M135. Org. Lett. 2012, 14, 6194-6197.

230. Warabi, K.; Matsunaga, S.; van Soest, R.W.M.; Fusetani, N. Dictyodendrins A-E, the First Telomerase-Inhibitory Marine Natural Products from the Sponge Dictyodendrilla verongiformis. J. Org. Chem. 2003, 68, 2765-2770.

231. Zhang, H.; Conte, M.M.; Khalil, Z.; Huang, X.-C.; Capon, R.J. New dictyodendrins as BACE inhibitors from a southern Australian marine sponge, Ianthella sp. RSC Advances 2012, 2, 4209-4214.

232. Iwagawa, T.; Miyazaki, M.; Okamura, H.; Nakatani, M.; Doe, M.; Takemura, K. Three novel bis(indole) alkaloids from a stony coral, Tubastraea sp. Tetrahedron Lett. 2003, 44, 2533-2535.

233. Balansa, W.; Islam, R.; Gilbert, D.F.; Fontaine, F.; Xiao, X.; Zhang, H.; Piggott, A.M.; Lynch, J.W.; Capon, R.J. Australian marine sponge alkaloids as a new class of glycine-gated chloride channel receptor modulator. Bioorg. Med. Chem. 2013, 21, 4420-4425.

234. Iwagawa, T.; Miyazaki, M.; Yokogawa, Y.; Okamura, H.; Nakatani, M.; Doe, M.; Morimoto, Y.; Takemura, K. Aplysinopsin dimers from a stony coral Tubastraea aurea. Heterocycles 2008, 75, 2023-2028.

235. Meyer, M.; Delberghe, F.; Liron, F.; Guillaume, M.; Valentin, A.; Guyot, M. An antiplasmodial new (bis)indole alkaloid from the hard coral Tubastraea sp. Nat. Prod. Res. 2009, 23, 178-182.

236. Tan, R.X.; Jensen, P.R.; Williams, P.G.; Fenical, W. Isolation and Structure Assignments of Rostratins A-D, Cytotoxic Disulfides Produced by the Marine-Derived Fungus Exserohilum rostratum. J. Nat. Prod. 2004, 67, 1374-1382.

237. Ovenden, S.P.B.; Sberna, G.; Tait, R.M.; Wildman, H.G.; Patel, R.; Li, B.; Steffy, K.; Nguyen, N.; Meurer-Grimes, B.M. A Diketopiperazine Dimer from a Marine-Derived Isolate of Aspergillus niger. J. Nat. Prod. 2004, 67, 2093-2095.

238. Oh, K.-B.; Mar, W.; Kim, S.; Kim, J.-Y.; Oh, M.-N.; Kim, J.-G.; Shin, D.; Sim, C.J.; Shin, J. Bis(indole) alkaloids as sortase A inhibitors from the sponge Spongosorites sp. Bioorg. Med. Chem. Lett. 2005, 15, 4927-4931.

239. Bao, B.; Sun, Q.; Yao, X.; Hong, J.; Lee, C.-O.; Sim, C.J.; Im, K.S.; Jung, J.H. Cytotoxic Bisindole Alkaloids from a Marine Sponge Spongosorites sp. J. Nat. Prod. 2005, 68, 711-715. 
240. Bao, B.; Sun, Q.; Yao, X.; Hong, J.; Lee, C.-O.; Cho, H.Y.; Jung, J.H. Bisindole Alkaloids of the Topsentin and Hamacanthin Classes from a Marine Sponge Spongosorites sp. J. Nat. Prod. 2007, $70,2-8$.

241. Oh, K.-B.; Mar, W.; Kim, S.; Kim, J.-Y.; Lee, T.-H.; Kim, J.-G.; Shin, D.; Sim, C.J.; Shin, J. Antimicrobial activity and cytotoxicity of bis(indole) alkaloids from the sponge Spongosorites sp. Biol. Pharm. Bull. 2006, 29, 570-573.

242. Kim, G.; Cheong, O.; Bae, S.; Shin, J.; Lee, S. 6"-Debromohamacanthin A, a Bis (Indole) Alkaloid, Inhibits Angiogenesis by Targeting the VEGFR2-Mediated PI3K/AKT/mTOR Signaling Pathways. Mar. Drugs 2013, 11, 1087-1103.

243. Sun, W.-S.; Su, S.; Zhu, R.-X.; Tu, G.-Z.; Cheng, W.; Liang, H.; Guo, X.-Y.; Zhao, Y.-Y.; Zhang, Q.-Y. A pair of unprecedented spiro-trisindole enantiomers fused through a five-member ring from Laurencia similis. Tetrahedron Lett. 2013, 54, 3617-3620.

244. Dai, J.; Jiménez, J.I.; Kelly, M.; Barnes, S.; Lorenzo, P.; Williams, P. Dictazolines A and B, Bisspiroimidazolidinones from the Marine Sponge Smenospongia cerebriformis. J. Nat. Prod. 2008, 71, 1287-1290.

245. Dai, J.; Jiménez, J.I.; Kelly, M.; Williams, P.G. Dictazoles: Potential Vinyl Cyclobutane Biosynthetic Precursors to the Dictazolines. J. Org. Chem. 2010, 75, 2399-2402.

246. Tapiolas, D.M.; Bowden, B.F.; Abou-Mansour, E.; Willis, R.H.; Doyle, J.R.; Muirhead, A.N.; Liptrot, C.; Llewellyn, L.E.; Wolff, C.W.W.; Wright, A.D.; et al. Eusynstyelamides A, B, and C, nNOS Inhibitors, from the Ascidian Eusynstyela latericius. J. Nat. Prod. 2009, 72, 1115-1120.

247. Tadesse, M.; Tabudravu, J.N.; Jaspars, M.; Strøm, M.B.; Hansen, E.; Andersen, J.H.; Kristiansen, P.E.; Haug, T. The Antibacterial ent-Eusynstyelamide B and Eusynstyelamides D, E, and F from the Arctic Bryozoan Tegella cf. spitzbergensis. J. Nat. Prod. 2011, 74, 837-841.

248. Liberio, M.; Sadowski, M.; Nelson, C.; Davis, R. Identification of Eusynstyelamide B as a Potent Cell Cycle Inhibitor Following the Generation and Screening of an Ascidian-Derived Extract Library Using a Real Time Cell Analyzer. Mar. Drugs 2014, 12, 5222-5239.

249. Liberio, M.; Sadowski, M.; Rockstroh, A.; Vasireddy, R.; Quinn, R.J.; Davis, R.A.; Nelson, C. Scientific Programme-Proffered Papers. Eur. J. Cancer 2013, 49 (Suppl. 2), S177-S178.

250. Raju, R.; Piggott, A.M.; Conte, M.; Aalbersberg, W.G.L.; Feussner, K.; Capon, R.J. Naseseazines A and B: A New Dimeric Diketopiperazine Framework from a Marine-Derived Actinomycete, Streptomyces sp. Org. Lett. 2009, 11, 3862-3865.

251. Carr, G.; Tay, W.; Bottriell, H.; Andersen, S.K.; Mauk, A.G.; Andersen, R.J. Plectosphaeroic Acids A, B, and C, Indoleamine 2,3-Dioxygenase Inhibitors Produced in Culture by a Marine Isolate of the Fungus Plectosphaerella cucumerina. Org. Lett. 2009, 11, 2996-2999.

252. Wei, X.; Henriksen, N.M.; Skalicky, J.J.; Harper, M.K.; Cheatham, T.E.; Ireland, C.M.; Van Wagoner, R.M. Araiosamines A-D: Tris-bromoindole Cyclic Guanidine Alkaloids from the Marine Sponge Clathria (Thalysias) araiosa. J. Org. Chem. 2011, 76, 5515-5523.

253. Cai, S.; Kong, X.; Wang, W.; Zhou, H.; Zhu, T.; Li, D.; Gu, Q. Aspergilazine A, a diketopiperazine dimer with a rare N-1 to C-6 linkage, from a marine-derived fungus Aspergillus taichungensis. Tetrahedron Lett. 2012, 53, 2615-2617; Corrigendum in 2014, 55, 5404. 
254. Wang, F.-Z.; Huang, Z.; Shi, X.-F.; Chen, Y.-C.; Zhang, W.-M.; Tian, X.-P.; Li, J.; Zhang, S. Cytotoxic indole diketopiperazines from the deep sea-derived fungus Acrostalagmus luteoalbus SCSIO F457. Bioorg. Med. Chem. Lett. 2012, 22, 7265-7267.

255. Liu, D.-Q.; Mao, S.-C.; Zhang, H.-Y.; Yu, X.-Q.; Feng, M.-T.; Wang, B.; Feng, L.-H.; Guo, Y.-W. Racemosins A and B, two novel bisindole alkaloids from the green alga Caulerpa racemosa. Fitoterapia 2013, 91, 15-20.

256. Yang, H.; Liu, D.-Q.; Liang, T.-J.; Li, J.; Liu, A.-H.; Yang, P.; Lin, K.; Yu, X.-Q.; Guo, Y.-W.; Mao, S.-C.; et al. Racemosin C, a novel minor bisindole alkaloid with protein tyrosine phosphatase-1B inhibitory activity from the green alga Caulerpa racemosa. J. Asian. Nat. Prod. Res. 2014, 16, 1158-1165.

257. Liu, D.-Q.; Mao, S.-C.; Yu, X.-Q.; Feng, L.-H.; Lai, X.-P. Caulerchlorin, a Novel Chlorinated Bisindole Alkaloid with Antifungal Activity from the Chinese Green Alga Caulerpa racemosa. Heterocycles 2012, 85, 661-666.

258. Momose, R.; Tanaka, N.; Fromont, J.; Kobayashi, J.I. Hyrtimomines A-C, New Heteroaromatic Alkaloids from a Sponge Hyrtios sp. Org. Lett. 2013, 15, 2010-2013.

259. Tanaka, N.; Momose, R.; Takahashi, Y.; Kubota, T.; Takahashi-Nakaguchi, A.; Gonoi, T.; Fromont, J.; Kobayashi, J.I. Hyrtimomines D and E, bisindole alkaloids from a marine sponge Hyrtios sp. Tetrahedron Lett. 2013, 54, 4038-4040.

260. Tanaka, N.; Momose, R.; Takahashi-Nakaguchi, A.; Gonoi, T.; Fromont, J.; Kobayashi, J.I. Hyrtimomines, indole alkaloids from Okinawan marine sponges Hyrtios spp. Tetrahedron 2014, 70, 832-837.

261. McArthur, K.A.; Mitchell, S.S.; Tsueng, G.; Rheingold, A.; White, D.J.; Grodberg, J.; Lam, K.S.; Potts, B.C.M. Lynamicins A-E, Chlorinated Bisindole Pyrrole Antibiotics from a Novel Marine Actinomycete. J. Nat. Prod. 2008, 71, 1732-1737.

262. Zhang, W.; Liu, Z.; Li, S.; Yang, T.; Zhang, Q.; Ma, L.; Tian, X.; Zhang, H.; Huang, C.; Zhang, S.; et al. Spiroindimicins A-D: New Bisindole Alkaloids from a Deep-Sea-Derived Actinomycete. Org. Lett. 2012, 14, 3364-3367.

263. Zhang, W.; Ma, L.; Li, S.; Liu, Z.; Chen, Y.; Zhang, H.; Zhang, G.; Zhang, Q.; Tian, X.; Yuan, C.; et al. Indimicins A-E, Bisindole Alkaloids from the Deep-Sea-Derived Streptomyces sp. SCSIO 03032. J. Nat. Prod. 2014, 77, 1887-1892.

264. Saurav, K.; Zhang, W.; Saha, S.; Zhang, H.; Li, S.; Zhang, Q.; Wu, Z.; Zhang, G.; Zhu, Y.; Verma, G. In silico molecular docking, preclinical evaluation of spiroindimicins A-D, lynamicin A and D isolated from deep marine sea derived Streptomyces sp. SCSIO 03032. Interdiscip. Sci. Comput. Life Sci. 2014, 6, 187-196.

265. Meng, L.-H.; Li, X.-M.; Lv, C.-T.; Huang, C.-G.; Wang, B.-G. Brocazines A-F, Cytotoxic Bisthiodiketopiperazine Derivatives from Penicillium brocae MA-231, an Endophytic Fungus Derived from the Marine Mangrove Plant Avicennia marina. J. Nat. Prod. 2014, 77, 1921-1927.

266. Reyes, F.; Martín, R.; Rueda, A.; Fernández, R.; Montalvo, D.; Gómez, C.; Sánchez-Puelles, J.M. Discorhabdins I and L, Cytotoxic Alkaloids from the Sponge Latrunculia brevis. J. Nat. Prod. 2004, 67, 463-465. 
267. Antunes, E.M.; Beukes, D.R.; Kelly, M.; Samaai, T.; Barrows, L.R.; Marshall, K.M.; Sincich, C.; Davies-Coleman, M.T. Cytotoxic Pyrroloiminoquinones from Four New Species of South African Latrunculid Sponges. J. Nat. Prod. 2004, 67, 1268-1276.

268. Lang, G.; Pinkert, A.; Blunt, J.W.; Munro, M.H.G. Discorhabdin W, the First Dimeric Discorhabdin. J. Nat. Prod. 2005, 68, 1796-1798.

269. El-Naggar, M.; Capon, R.J. Discorhabdins Revisited: Cytotoxic Alkaloids from Southern Australian Marine Sponges of the Genera Higginsia and Spongosorites. J. Nat. Prod. 2009, 72, 460-464.

270. Na, M.; Ding, Y.; Wang, B.; Tekwani, B.L.; Schinazi, R.F.; Franzblau, S.; Kelly, M.; Stone, R.; Li, X.-C.; Ferreira, D.; et al. Anti-infective Discorhabdins from a Deep-Water Alaskan Sponge of the Genus Latrunculia. J. Nat. Prod. 2010, 73, 383-387.

271. Jeon, J.-E.; Na, Z.; Jung, M.; Lee, H.-S.; Sim, C.J.; Nahm, K.; Oh, K.-B.; Shin, J. Discorhabdins from the Korean Marine Sponge Sceptrella sp. J. Nat. Prod. 2010, 73, 258-262.

272. Davis, R.A.; Buchanan, M.S.; Duffy, S.; Avery, V.M.; Charman, S.A.; Charman, W.N.; White, K.L.; Shackleford, D.M.; Edstein, M.D.; Andrews, K.T.; et al. Antimalarial Activity of Pyrroloiminoquinones from the Australian Marine Sponge Zyzzya sp. J. Med. Chem. 2012, 55, 5851-5858.

273. Keyzers, R.A.; Samaai, T.; Davies-Coleman, M.T. Novel pyrroloquinoline ribosides from the South African latrunculid sponge Strongylodesma aliwaliensis. Tetrahedron Lett. 2004, 45, 9415-9418.

274. Whibley, C.E.; Keyzers, R.A.; Soper, A.G.; Davies-Coleman, M.T.; Samaai, T.; Hendricks, D.T. Antiesophageal Cancer Activity from Southern African Marine Organisms. Ann. N. Y. Acad. Sci. 2005, 1056, 405-412.

275. Utkina, N.K.; Makarchenko, A.E.; Denisenko, V.A.; Dmitrenok, P.S. Zyzzyanone A, a novel pyrrolo[3,2-f]indole alkaloid from the Australian marine sponge Zyzzya fuliginosa. Tetrahedron Lett. 2004, 45, 7491-7494.

276. Utkina, N.K.; Makarchenko, A.E.; Denisenko, V.A. Zyzzyanones B-D, Dipyrroloquinones from the Marine Sponge Zyzzya fuliginosa. J. Nat. Prod. 2005, 68, 1424-1427.

277. Makarchenko, A.E.; Utkina, N.K. UV-stability and UV-protective activity of alkaloids from the marine sponge Zyzzya fuliginosa. Chem Nat Compd 2006, 42, 78-81.

278. Utkina, N.K.; Denisenko, V.A. Ophiuroidine, the first indolo[2,1-b]quinazoline alkaloid from the Caribbean brittle star Ophiocoma riisei. Tetrahedron Lett. 2007, 48, 4445-4447.

279. Han, X.-X.; Xu, X.-Y.; Cui, C.-B.; Gu, Q.-Q. Alkaloidal compounds produced by a marine-derived fungus, Aspergillus fumigatus H1-04, and their antitumor activities. Chin. J. Med. Chem. 2007, 17, 232-237.

280. Zhou, Y.; Debbab, A.; Mándi, A.; Wray, V.; Schulz, B.; Müller, W.E.G.; Kassack, M.; Lin, W.; Kurtán, T.; Proksch, P.; et al. Alkaloids from the Sponge-Associated Fungus Aspergillus sp. Eur. J. Org. Chem. 2013, 2013, 894-906.

281. Afiyatullov, S.S.; Zhuravleva, O.I.; Antonov, A.S.; Kalinovsky, A.I.; Pivkin, M.V.; Menchinskaya, E.S.; Aminin, D.L. New metabolites from the marine-derived fungus Aspergillus fumigatus. Nat. Prod. Commun. 2012, 7, 497-500. 
282. Shao, C.-L.; Xu, R.-F.; Wei, M.-Y.; She, Z.-G.; Wang, C.-Y. Structure and Absolute Configuration of Fumiquinazoline L, an Alkaloid from a Gorgonian-Derived Scopulariopsis sp. Fungus. J. Nat. Prod. 2013, 76, 779-782.

283. Liao, L.; You, M.; Chung, B.K.; Oh, D.-C.; Oh, K.-B.; Shin, J. Alkaloidal Metabolites from a Marine-Derived Aspergillus sp. Fungus. J. Nat. Prod. 2015, 78, 349-354.

284. Fremlin, L.J.; Piggott, A.M.; Lacey, E.; Capon, R.J. Cottoquinazoline A and Cotteslosins A and B, Metabolites from an Australian Marine-Derived Strain of Aspergillus versicolor. J. Nat. Prod. 2009, 72, 666-670.

285. Zhuang, Y.; Teng, X.; Wang, Y.; Liu, P.; Li, G.; Zhu, W. New Quinazolinone Alkaloids within Rare Amino Acid Residue from Coral-Associated Fungus, Aspergillus versicolor LCJ-5-4. Org. Lett. 2011, 13, 1130-1133.

286. Liu, J.; Wei, X.; La Kim, E.; Lin, X.; Yang, X.-W.; Zhou, X.; Yang, B.; Jung, J.H.; Liu, Y. New glucosidated pyrazinoquinazoline indole alkaloids from fungus Aspergillus fumigatus derived of a jellyfish. Tetrahedron 2015, 71, 271-275.

287. Qi, S.-H.; Su, G.-C.; Wang, Y.-F.; Liu, Q.-Y.; Gao, C.-H. Alkaloids from the South China Sea Black Coral Antipathes dichotoma. Chem. Pharm. Bull. 2009, 57, 87-88.

288. Wu, Q.-X.; Crews, M.S.; Draskovic, M.; Sohn, J.; Johnson, T.A.; Tenney, K.; Valeriote, F.A.; Yao, X.-J.; Bjeldanes, L.F.; Crews, P. Azonazine, a Novel Dipeptide from a Hawaiian Marine Sediment-Derived Fungus, Aspergillus insulicola. Org. Lett. 2010, 12, 4458-4461.

289. Lee, S.U.; Asami, Y.; Lee, D.; Jang, J.-H.; Ahn, J.S.; Oh, H. Protuboxepins A and B and Protubonines A and B from the Marine-Derived Fungus Aspergillus sp. SF-5044. J. Nat. Prod. 2011, 74, 1284-1287.

290. An, C.-Y.; Li, X.-M.; Li, C.-S.; Wang, M.-H.; Xu, G.-M.; Wang, B.-G. Aniquinazolines A-D, Four New Quinazolinone Alkaloids from Marine-Derived Endophytic Fungus Aspergillus nidulans. Mar. Drugs 2013, 11, 2682.

291. Lyakhova, E.G.; Kolesnikova, S.A.; Kalinovsky, A.I.; Afiyatullov, S.S.; Dyshlovoy, S.A.; Krasokhin, V.B.; Minh, C.V.; Stonik, V.A. Bromine-containing alkaloids from the marine sponge Penares sp. Tetrahedron Lett. 2012, 53, 6119-6122.

292. Xu, N.; Cao, Y.; Wang, L.; Chen, G.; Pei, Y.-H. New alkaloids from a marine-derived fungus Neosartorya sp. HN-M-3. J. Asian. Nat. Prod. Res. 2013, 15, 731-736.

293. Sun, F.-Y.; Chen, G.; Bai, J.; Li, W.; Pei, Y.-H. Two new alkaloids from a marine-derived fungus Neosartorya sp. HN-M-3. J. Asian. Nat. Prod. Res. 2012, 14, 1109-1115.

294. Khokhar, S.; Feng, Y.; Campitelli, M.R.; Quinn, R.J.; Hooper, J.N.A.; Ekins, M.G.; Davis, R.A. Trikentramides A-D, Indole Alkaloids from the Australian Sponge Trikentrion flabelliforme. J. Nat. Prod. 2013, 76, 2100-2105.

295. Dong, J.-J.; Bao, J.; Zhang, X.-Y.; Xu, X.-Y.; Nong, X.-H.; Qi, S.-H. Alkaloids and citrinins from marine-derived fungus Nigrospora oryzae SCSGAF 0111. Tetrahedron Lett. 2014, 55, 2749-2753.

296. Yamanokuchi, R.; Imada, K.; Miyazaki, M.; Kato, H.; Watanabe, T.; Fujimuro, M.; Saeki, Y.; Yoshinaga, S.; Terasawa, H.; Iwasaki, N.; et al. Hyrtioreticulins A-E, indole alkaloids inhibiting the ubiquitin-activating enzyme, from the marine sponge Hyrtios reticulatus. Bioorg. Med. Chem. 2012, 20, 4437-4442. 
297. Imada, K.; Sakai, E.; Kato, H.; Kawabata, T.; Yoshinaga, S.; Nehira, T.; Terasawa, H.; Tsukamoto, S. Reticulatins A and B and hyrtioreticulin F from the marine sponge Hyrtios reticulatus. Tetrahedron 2013, 69, 7051-7055.

298. Ji, N.-Y.; Liu, X.-H.; Miao, F.-P.; Qiao, M.-F. Aspeverin, a New Alkaloid from an Algicolous Strain of Aspergillus versicolor. Org. Lett. 2013, 15, 2327-2329.

299. Fu, P.; Kong, F.; Li, X.; Wang, Y.; Zhu, W. Cyanogramide with a New Spiro[indolinonepyrroloimidazole] Skeleton from Actinoalloteichus cyanogriseus. Org. Lett. 2014, 16, 3708-3711.

300. Liu, Y.; Li, X.-M.; Meng, L.-H.; Wang, B.-G. $N$-Formyllapatin A, a new $N$-formylspiroquinazoline derivative from the marine-derived fungus Penicillium adametzioides AS-53. Phytochem. Lett. 2014, 10, 145-148.

301. Lee, C.; Sohn, J.H.; Jang, J.-H.; Ahn, J.S.; Oh, H.; Baltrusaitis, J.; Hwang, I.H.; Gloer, J.B. Cycloexpansamines A and B: Spiroindolinone alkaloids from a marine isolate of Penicillium sp. (SF-5292). J. Antibiot. 2015, doi:10.1038/ja.2015.56.

302. Choi, E.J.; Park, J.S.; Kim, Y.J.; Jung, J.H.; Lee, J.K.; Kwon, H.C.; Yang, H.O. Apoptosis-inducing effect of diketopiperazine disulfides produced by Aspergillus sp. KMD 901 isolated from marine sediment on HCT116 colon cancer cell lines. J. Appl. Microbiol. 2011, 110, 304-313.

303. Li, X.; Kim, S.-K.; Nam, K.W.; Kang, J.S.; Choi, H.D.; Son, B.W. A New Antibacterial Dioxopiperazine Alkaloid Related to Gliotoxin from a Marine Isolate of the Fungus Pseudallescheria. J. Antibiot. 2006, 59, 248-250.

304. Sun, Y.; Takada, K.; Takemoto, Y.; Yoshida, M.; Nogi, Y.; Okada, S.; Matsunaga, S. Gliotoxin Analogues from a Marine-Derived Fungus, Penicillium sp., and Their Cytotoxic and Histone Methyltransferase Inhibitory Activities. J. Nat. Prod. 2012, 75, 111-114.

305. Kong, F.; Wang, Y.; Liu, P.; Dong, T.; Zhu, W. Thiodiketopiperazines from the Marine-Derived Fungus Phoma sp. OUCMDZ-1847. J. Nat. Prod. 2014, 77, 132-137.

306. Cao, R.; Peng, W.; Wang, Z.; Xu, A. $\beta$-Carboline Alkaloids: Biochemical and Pharmacological Functions. Curr. Med. Chem. 2007, 14, 479-500.

307. Charan, R.D.; McKee, T.C.; Gustafson, K.R.; Pannell, L.K.; Boyd, M.R. Thorectandramine, a novel $\beta$-carboline alkaloid from the marine sponge Thorectandra sp. Tetrahedron Lett. 2002, 43, 5201-5204.

308. Segraves, N.L.; Lopez, S.; Johnson, T.A.; Said, S.A.; Fu, X.; Schmitz, F.J.; Pietraszkiewicz, H.; Valeriote, F.A.; Crews, P. Structures and cytotoxicities of fascaplysin and related alkaloids from two marine phyla-Fascaplysinopsis sponges and Didemnum tunicates. Tetrahedron Lett. 2003, 44, 3471-3475.

309. Kuzmich, A.S.; Fedorov, S.N.; Shastina, V.V.; Shubina, L.K.; Radchenko, O.S.; Balaneva, N.N.; Zhidkov, M.E.; Park, J.-I.; Kwak, J.Y.; Stonik, V.A. The anticancer activity of 3- and 10-bromofascaplysins is mediated by caspase-8, -9, -3-dependent apoptosis. Bioorg. Med. Chem. 2010, 18, 3834-3840.

310. Charan, R.D.; McKee, T.C.; Boyd, M.R. Cytotoxic Alkaloids from the Marine Sponge Thorectandra sp. Nat. Prod. Res. 2004, 18, 225-229.

311. Segraves, N.L.; Robinson, S.J.; Garcia, D.; Said, S.A.; Fu, X.; Schmitz, F.J.; Pietraszkiewicz, H.; Valeriote, F.A.; Crews, P. Comparison of Fascaplysin and Related Alkaloids: A Study of Structures, Cytotoxicities, and Sources. J. Nat. Prod. 2004, 67, 783-792. 
312. Ravinder, K.; Vijender Reddy, A.; Krishnaiah, P.; Ramesh, P.; Ramakrishna, S.; Laatsch, H.; Venkateswarlu, Y. Isolation and synthesis of a novel $\beta$-carboline guanidine derivative tiruchanduramine from the Indian ascidian Synoicum macroglossum. Tetrahedron Lett. 2005, 46, 5475-5478.

313. Peng, J.; Hu, J.-F.; Kazi, A.B.; Li, Z.; Avery, M.; Peraud, O.; Hill, R.T.; Franzblau, S.G.; Zhang, F.; Schinazi, R.F.; et al. Manadomanzamines A and B: A Novel Alkaloid Ring System with Potent Activity against Mycobacteria and HIV-1. J. Am. Chem. Soc. 2003, 125, 13382-13386.

314. Yousaf, M.; Hammond, N.L.; Peng, J.; Wahyuono, S.; McIntosh, K.A.; Charman, W.N.; Mayer, A.M.S.; Hamann, M.T. New Manzamine Alkaloids from an Indo-Pacific Sponge. Pharmacokinetics, Oral Availability, and the Significant Activity of Several Manzamines against HIV-I, AIDS Opportunistic Infections, and Inflammatory Diseases. J. Med. Chem. 2004, 47, 3512-3517.

315. Rao, K.V.; Kasanah, N.; Wahyuono, S.; Tekwani, B.L.; Schinazi, R.F.; Hamann, M.T. Three New Manzamine Alkaloids from a Common Indonesian Sponge and Their Activity against Infectious and Tropical Parasitic Diseases. J. Nat. Prod. 2004, 67, 1314-1318.

316. Rao, K.V.; Donia, M.S.; Peng, J.; Garcia-Palomero, E.; Alonso, D.; Martinez, A.; Medina, M.; Franzblau, S.G.; Tekwani, B.L.; Khan, S.I.; et al. Manzamine B and E and Ircinal A Related Alkaloids from an Indonesian Acanthostrongylophora Sponge and Their Activity against Infectious, Tropical Parasitic, and Alzheimer's Diseases. J. Nat. Prod. 2006, 69, 1034-1040.

317. Takahashi, Y.; Kubota, T.; Fromont, J.; Kobayashi, J.I. Zamamidines A and B, New Manzamine Alkaloids from the Sponge Amphimedon Species. Org. Lett. 2009, 11, 21-24.

318. Yamada, M.; Takahashi, Y.; Kubota, T.; Fromont, J.; Ishiyama, A.; Otoguro, K.; Yamada, H.; Ōmura, S.; Kobayashi, J.I. Zamamidine C, 3,4-dihydro-6-hydroxy-10,11-epoxymanzamine A, and 3,4-dihydromanzamine $\mathrm{J} N$-oxide, new manzamine alkaloids from sponge Amphimedon sp. Tetrahedron 2009, 65, 2313-2317; Corrigendum in 2009, 65, 6263.

319. Wahba, A.E.; Fromentin, Y.; Zou, Y.; Hamann, M.T. Acantholactone, a new manzamine related alkaloid with an unprecedented $\delta$-lactone and $\varepsilon$-lactam ring system. Tetrahedron Lett. 2012, 53, 6329-6331.

320. El-Desoky, A.H.; Kato, H.; Eguchi, K.; Kawabata, T.; Fujiwara, Y.; Losung, F.; Mangindaan, R.E.P.; de Voogd, N.J.; Takeya, M.; Yokosawa, H.; et al. Acantholactam and Pre-neo-kauluamine, Manzamine-Related Alkaloids from the Indonesian Marine Sponge Acanthostrongylophora ingens. J. Nat. Prod. 2014, 77, 1536-1540.

321. Furusato, A.; Kato, H.; Nehira, T.; Eguchi, K.; Kawabata, T.; Fujiwara, Y.; Losung, F.; Mangindaan, R.E.P.; de Voogd, N.J.; Takeya, M.; et al. Acanthomanzamines A-E with New Manzamine Frameworks from the Marine Sponge Acanthostrongylophora ingens. Org. Lett. 2014, 16, 3888-3891.

322. Pedpradab, S.; Edrada, R.; Ebel, R.; Wray, V.; Proksch, P. New $\beta$-Carboline Alkaloids from the Andaman Sea Sponge Dragmacidon sp. J. Nat. Prod. 2004, 67, 2113-2116.

323. Iinuma, Y.; Kozawa, S.; Ishiyama, H.; Tsuda, M.; Fukushi, E.; Kawabata, J.; Fromont, J.; Kobayashi, J.I. Gesashidine A, a $\beta$-Carboline Alkaloid with an Imidazole Ring from a Thorectidae Sponge. J. Nat. Prod. 2005, 68, 1109-1110. 
324. Ibrahim, S.R.M.; Ebel, R.; Ebel, R.; Proksch, P. Acanthomine A, a new pyrimidine- $\beta$-carboline alkaloid from the sponge Acanthostrongylophora ingens. Nat. Prod. Commun. 2008, 3, 175-178.

325. Till, M.; Prinsep, M.R. 5-Bromo-8-methoxy-1-methyl- $\beta$-carboline, an Alkaloid from the New Zealand Marine Bryozoan Pterocella vesiculosa. J. Nat. Prod. 2009, 72, 796-798.

326. Wang, W.; Nam, S.-J.; Lee, B.-C.; Kang, H. $\beta$-Carboline Alkaloids from a Korean Tunicate Eudistoma sp. J. Nat. Prod. 2008, 71, 163-166.

327. Takahashi, Y.; Ishiyama, H.; Kubota, T.; Kobayashi, J.I. Eudistomidin G, a new $\beta$-carboline alkaloid from the Okinawan marine tunicate Eudistoma glaucus and structure revision of eudistomidin B. Bioorg. Med. Chem. Lett. 2010, 20, 4100-4103.

328. Suzuki, T.; Kubota, T.; Kobayashi, J.I. Eudistomidins H-K, new $\beta$-carboline alkaloids from the Okinawan marine tunicate Eudistoma glaucus. Bioorg. Med. Chem. Lett. 2011, 21, 4220-4223.

329. Kearns, P.S.; Rideout, J.A. Nonsymmetrical $\beta$-Carboline Dimers from an Ascidian, Didemnum sp. J. Nat. Prod. 2008, 71, 1280-1282.

330. Lee, H.-S.; Yoon, K.-M.; Han, Y.-R.; Lee, K.J.; Chung, S.-C.; Kim, T.-I.; Lee, S.-H.; Shin, J.; Oh, K.-B. 5-Hydroxyindole-type alkaloids, as Candida albicans isocitrate lyase inhibitors, from the tropical sponge Hyrtios sp. Bioorg. Med. Chem. Lett. 2009, 19, 1051-1053.

331. Youssef, D.; Shaala, L.; Asfour, H. Bioactive Compounds from the Red Sea Marine Sponge Hyrtios Species. Mar. Drugs 2013, 11, 1061-1070.

332. Inman, W.D.; Bray, W.M.; Gassner, N.C.; Lokey, R.S.; Tenney, K.; Shen, Y.Y.; TenDyke, K.; Suh, T.; Crews, P. A $\beta$-Carboline Alkaloid from the Papua New Guinea Marine Sponge Hyrtios reticulatus. J. Nat. Prod. 2010, 73, 255-257.

333. Khokhar, S.; Feng, Y.; Campitelli, M.R.; Ekins, M.G.; Hooper, J.N.A.; Beattie, K.D.; Sadowski, M.C.; Nelson, C.C.; Davis, R.A. Isolation, structure determination and cytotoxicity studies of tryptophan alkaloids from an Australian marine sponge Hyrtios sp. Bioorg. Med. Chem. Lett. 2014, 24, 3329-3332.

334. Ren, S.; Ma, W.; Xu, T.; Lin, X.; Yin, H.; Yang, B.; Zhou, X.-F.; Yang, X.-W.; Long, L.; Lee, K.J.; et al. Two novel alkaloids from the South China Sea marine sponge Dysidea sp. J. Antibiot. 2010, 63, 699-701.

335. Qi, S.-H.; Miao, L.; Gao, C.-H.; Xu, Y.; Zhang, S.; Qian, P.-Y. New Steroids and a New Alkaloid from the Gorgonian Isis minorbrachyblasta: Structures, Cytotoxicity, and Antilarval Activity. Helvetica Chimica Acta 2010, 93, 511-516.

336. Davis, R.A.; Duffy, S.; Avery, V.M.; Camp, D.; Hooper, J.N.A.; Quinn, R.J. $(+)$-7-Bromotrypargine: An antimalarial $\beta$-carboline from the Australian marine sponge Ancorina sp. Tetrahedron Lett. 2010, 51, 583-585.

337. Chan, S.T.S.; Pearce, A.N.; Page, M.J.; Kaiser, M.; Copp, B.R. Antimalarial $\beta$-Carbolines from the New Zealand Ascidian Pseudodistoma opacum. J. Nat. Prod. 2011, 74, 1972-1979. 
338. Savi, D.; Shaaban, K.; Vargas, N.; Ponomareva, L.; Possiede, Y.; Thorson, J.; Glienke, C.; Rohr, J. Microbispora sp. LGMB259 Endophytic Actinomycete Isolated from Vochysia divergens (Pantanal, Brazil) Producing $\beta$-Carbolines and Indoles with Biological Activity. Curr. Microbiol. 2015, 70, 345-354.

(C) 2015 by the authors; licensee MDPI, Basel, Switzerland. This article is an open access article distributed under the terms and conditions of the Creative Commons Attribution license (http://creativecommons.org/licenses/by/4.0/). 